ESAIM: COCV 20 (2014) 1078-1122

DOI: $10.1051 / \mathrm{cocv} / 2014008$
ESAIM: Control, Optimisation and Calculus of Variations

www.esaim-cocv.org

\title{
RELAXATION IN BV OF INTEGRALS WITH SUPERLINEAR GROWTH
}

\author{
PARTH SONEJI ${ }^{1}$
}

Abstract. We study properties of the functional

$$
\mathscr{F}_{\mathrm{loc}}(u, \Omega):=\inf _{\left(u_{j}\right)}\left\{\begin{array}{l|l}
\liminf _{j \rightarrow \infty} \int_{\Omega} f\left(\nabla u_{j}\right) \mathrm{d} x \mid \begin{array}{l}
\left(u_{j}\right) \subset W_{\mathrm{loc}}^{1, r}\left(\Omega, \mathbb{R}^{N}\right) \\
u_{j} \stackrel{*}{*} u \operatorname{in} \operatorname{BV}\left(\Omega, \mathbb{R}^{N}\right)
\end{array}
\end{array}\right\},
$$

where $u \in \operatorname{BV}\left(\Omega ; \mathbb{R}^{N}\right)$, and $f: \mathbb{R}^{N \times n} \rightarrow \mathbb{R}$ is continuous and satisfies $0 \leq f(\xi) \leq L\left(1+|\xi|^{r}\right)$. For $r \in[1,2)$, assuming $f$ has linear growth in certain rank-one directions, we combine a result of $[$ A. Braides and A. Coscia, Proc. Roy. Soc. Edinburgh Sect. A 124 (1994) 737-756] with a new technique involving mollification to prove an upper bound for $\mathscr{F}_{\text {loc }}$. Then, for $r \in\left[1, \frac{n}{n-1}\right)$, we prove that $\mathscr{F}_{\text {loc }}$ satisfies the lower bound

$$
\mathscr{F}_{\text {loc }}(u, \Omega) \geq \int_{\Omega} f(\nabla u(x)) \mathrm{d} x+\int_{\Omega} f^{\infty}\left(\frac{D^{s} u}{\left|D^{s} u\right|}\right)\left|D^{s} u\right|,
$$

provided $f$ is quasiconvex, and the recession function $f^{\infty}$ (defined as $f^{\infty}(\xi):=\varlimsup_{\lim } \rightarrow \infty f(t \xi) / t$ ) is assumed to be finite in certain rank-one directions. The proof of this result involves adapting work by [Kristensen, Calc. Var. Partial Differ. Eqs. 7 (1998) 249-261], and [Ambrosio and Dal Maso, J. Funct. Anal. 109 (1992) 76-97], and applying a non-standard blow-up technique that exploits fine properties of BV maps. It also makes use of the fact that $\mathscr{F}_{\text {loc }}$ has a measure representation, which is proved in the appendix using a method of [Fonseca and Malý, Annal. Inst. Henri Poincaré Anal. Non Linéaire 14 (1997) 309-338].

Mathematics Subject Classification. 49J45, 26B30.

Received July 22, 2013. Revised November 27, 2013.

Published online August 13, 2014.

\section{INTRODUCTION}

Consider the variational integral

$$
F(u, \Omega):=\int_{\Omega} f(\nabla u(x)) \mathrm{d} x,
$$

where $\Omega$ is a bounded, open subset of $\mathbb{R}^{n}, n \geq 2, u: \Omega \rightarrow \mathbb{R}^{N}$ is a vector-valued function, $\nabla u$ denotes the Jacobian matrix of $u$ and $f$ is a non-negative continuous function defined in the space $\mathbb{R}^{N \times n}$ of all real $N \times n$

Keywords and phrases. Quasiconvexity, lower semicontinuity, relaxation, BV.

1 Ludwig Maximilians University Munich, Theresienstr. 39, 80333 Munich, Germany. soneji@math.lmu.de 
matrices. Throughout this paper, we shall also assume that $f$ satisfies the growth condition

$$
0 \leq f(\xi) \leq L\left(1+|\xi|^{r}\right)
$$

for all $\xi \in \mathbb{R}^{N \times n}$, for some constant $L>0$, and some exponent $1 \leq r<\infty$.

Note that it is not obvious how to define $F(u, \Omega)$ when $u$ is a generic function of Bounded Variation. Following a method that was first used by Lebesgue for the area integral [32], and then adopted by Serrin [46,47] and, in the modern context, by Marcellini [35], we may extend the definition of $F(u, \Omega)$ by introducing the functionals

$$
\mathscr{F}(u, \Omega):=\inf _{\left(u_{j}\right)}\left\{\begin{array}{l|l}
\liminf _{j \rightarrow \infty} \int_{\Omega} f\left(\nabla u_{j}\right) \mathrm{d} x & \begin{array}{l}
\left(u_{j}\right) \subset W^{1, r}\left(\Omega, \mathbb{R}^{N}\right) \\
u_{j} \stackrel{*}{*} u \operatorname{in} \operatorname{BV}\left(\Omega, \mathbb{R}^{N}\right)
\end{array}
\end{array}\right\}
$$

and

$$
\mathscr{F}_{\text {loc }}(u, \Omega):=\inf _{\left(u_{j}\right)}\left\{\begin{array}{l|l}
\liminf _{j \rightarrow \infty} \int_{\Omega} f\left(\nabla u_{j}\right) \mathrm{d} x & \begin{array}{l}
\left(u_{j}\right) \subset W_{\text {loc }}^{1, r}\left(\Omega, \mathbb{R}^{N}\right) \\
u_{j} * *
\end{array} u \operatorname{in} \operatorname{BV}\left(\Omega, \mathbb{R}^{N}\right)
\end{array}\right\} .
$$

These are known as Lebesgue-Serrin Extensions of $F$, and are important quantities not only when we want to define $F(u, \Omega)$ for a wider class of functions $u$ but also, for example, when there is a lack of convexity.

Firstly suppose $r \in[1,2)$, and $f$ has linear growth for matrices $\xi=\eta \otimes \nu$, where $\eta \in \operatorname{span}\{u(y): y \in \Omega\}$, $\nu \in \mathbb{R}^{N}$. We shall show that $\mathscr{F}_{\text {loc }}$ satisfies the upper bound

$$
\mathscr{F}_{\text {loc }}(u, \Omega) \leq C\left(\mathscr{L}^{n}(\Omega)+|D u|(\Omega)\right) .
$$

We do this by first obtaining the upper bound for specific types of functions of Special Bounded Variation via mollification and a covering argument, and then using a technique Braides and Coscia to extend this result to general functions of Bounded Variation.

Now suppose in addition that $f$ is quasiconvex. That is, it satisfies

$$
\int_{\mathbb{R}^{n}}[f(\xi+\nabla \phi(x))-f(\xi)] \mathrm{d} x \geq 0
$$

for all $\xi \in \mathbb{R}^{N \times n}$ and all test functions $\phi \in W_{0}^{1, \infty}\left(\mathbb{R}^{n} ; \mathbb{R}^{N}\right)$. Then, for $r \in\left[1, \frac{n}{n-1}\right)$, we prove that $\mathscr{F}_{\text {loc }}$ satisfies the lower bound

$$
\mathscr{F}_{\text {loc }}(u, \Omega) \geq \int_{\Omega} f(\nabla u(x)) \mathrm{d} x+\int_{\Omega} f^{\infty}\left(\frac{\mathrm{d} D^{s} u}{\mathrm{~d}\left|D^{s} u\right|}\right) \mathrm{d}\left|D^{s} u\right|
$$

where $\nabla u$ is the density of the absolutely continuous part of the measure $D u$ with respect to Lebesgue measure, $D^{s} u$ is the singular part of $D u, \frac{d D^{s} u}{\mathrm{~d}\left|D^{s} u\right|}$ is the Radon-Nikodým derivative of the measure $D^{s} u$ with respect to its variation $\left|D^{s} u\right|$, and $f^{\infty}$ denotes the recession function of $f$, defined as

$$
f^{\infty}(\xi):=\limsup _{t \rightarrow \infty} \frac{f(t \xi)}{t} .
$$

In order to obtain this result, we need to assume additionally that $f^{\infty}$ is finite in certain rank-one directions. That is, for a given $u \in \operatorname{BV}\left(\Omega ; \mathbb{R}^{N}\right)$,

$$
f^{\infty}(u(y) \otimes \nu)<\infty \quad \text { for } \mathscr{L}^{n} \text {-a.a. } y \in \Omega \text { and all } \nu \in \mathbb{R}^{n} .
$$

This is a natural assumption, since otherwise $f^{\infty}\left(d D^{s} u / \mathrm{d}\left|D^{s} u\right|\right)$ may just be infinity for general BV functions.

In fact, the results in this paper also enable us to show that there can be no non-negative quasiconvex function of genuinely $r$ growth for $1<r<\frac{n}{n-1}$ for which $f^{\infty}$ is finite in all rank-one directions. That is, if $f$ is quasiconvex and satisfies (1.2) for $1 \leq r<\frac{n}{n-1}$ but has linear growth for all matrices $\xi$ where $\operatorname{rank}(\xi) \leq 1$, then $f$ must in fact have linear growth in all directions. 
The proof of this latter lower semicontinuity result also requires us to have shown that when $r \in\left[1, \frac{n}{n-1}\right)$, the functional $\mathscr{F}_{\text {loc }}(u, \cdot)$, if finite, is representable by a Radon measure on $\Omega$. This involves adapting a result of Fonseca and Malý in [24], and is proved in the appendix.

Throughout the course of this paper, we shall use $C$ to denote a generic positive constant which may not be the same from line to line. We shall specify what $C$ is dependent on in cases where it may not be entirely clear. Moreover for conciseness we will often just refer to the Radon-Nikodým derivative $\frac{\mathrm{d} D^{s} u}{\mathrm{~d}\left|D^{s} u\right|}$ of a BV map $u$ as simply $\frac{D^{s} u}{\left|D^{s} u\right|}$ (and similarly $\frac{D u}{\mid D u}$ ), and likewise we will often just write $\left|D^{s} u\right|$ instead of $\mathrm{d}\left|D^{s} u\right|$ when integrating with respect to this measure.

We shall now provide a short background for the results contained in this paper.

\subsection{Lower semicontinuity and relaxation in Sobolev spaces}

The classical lower semicontinuity result for quasiconvex integrands states that if the integrand $f$ is quasiconvex and satisfies (1.2) for some exponent $r$, then $F$ is lower semincontinuous in the sequential weak topology of $W^{1, r}\left(\Omega ; \mathbb{R}^{N}\right)$. This was first proved by Morrey without growth conditions, in the setting of $W^{1, \infty}\left(\Omega ; \mathbb{R}^{N}\right)$ and weak* convergence [38,39], with refinements made most notably by Meyers [37], Acerbi and Fusco [1], and Marcellini [34].

More recently, progress has been made to refine this result by considering convergence in Sobolev Spaces below the growth exponent of $f$ : keeping the same assumption of quasiconvexity and the growth condition (1.2), sequential weak lower semicontinuity in $W^{1, q}\left(\Omega ; \mathbb{R}^{N}\right)$ for $q>r \frac{n-1}{n}(q>1)$ was proved by Fonseca and Malý in [24,33]. Previously, work by Marcellini in [35], and by Carbone and De Arcangelis in [18] established lower semicontinuity for $q>r \frac{n}{n+1}$ by imposing additional structural conditions on $f$. Fonseca and Marcellini obtained a proof in the case $q>r-1$ [25], and Malý for $q \geq r-1$ : both these results require further assumptions on $f$ in addition to quasiconvexity.

Let us consider the minimisation problem

$$
m:=\inf \left\{\int_{\Omega} f(\nabla v(x)) \mathrm{d} x: v \in W^{1, r}\left(\Omega ; \mathbb{R}^{N}\right), v=g \text { on } \partial \Omega\right\} .
$$

As is well known, lower semicontinuity results relate straightforwardly to the problem of existence of minimisers when we assume that the integrand $f$ satisfies a " $q$-coercivity" property such as

$$
f(\xi) \geq c_{0}|\xi|^{q}-c_{1}
$$

for all $\xi \in \mathbb{R}^{N \times n}$, when $q \geq r$. We remark that such coercivity conditions may in fact be weakened for such purposes. However, when $q<r$ it is possible that the limit map $u \in W^{1, q}\left(\Omega ; \mathbb{R}^{N}\right)$ of a minimising sequence $\left(u_{j}\right) \subset W^{1, r}\left(\Omega ; \mathbb{R}^{N}\right)$, although it satisfies

$$
\int_{\Omega} f(\nabla u) \mathrm{d} x \leq \int_{\Omega} f(\nabla v) \mathrm{d} x \text { for all } v \in W^{1, r}\left(\Omega ; \mathbb{R}^{N}\right),
$$

is not in $W^{1, r}\left(\Omega ; \mathbb{R}^{N}\right)$ and hence not a solution of (1.5). Indeed, due to the Lavrentiev Phenomenon, it need not even satisfy the related minimisation problem to (1.5) for the case where admissible maps can be in the larger space $W^{1, q}\left(\Omega ; \mathbb{R}^{N}\right)$. That is, it is possible that

$$
\int_{\Omega} f(\nabla u) \mathrm{d} x>\inf \left\{\int_{\Omega} f(\nabla v(x)) \mathrm{d} x: v \in W^{1, q}\left(\Omega ; \mathbb{R}^{N}\right), v=g \text { on } \partial \Omega\right\} .
$$

In this case, we may relax the problem (1.5): following a similar method to the definition of $\mathscr{F}_{\text {loc }}$ above, for $u \in W^{1, q}\left(\Omega ; \mathbb{R}^{N}\right)$ we may take the Lebesgue-Serrin Extension

$$
\mathscr{F}^{r, q}(u, \Omega):=\inf _{\left(u_{j}\right)}\left\{\begin{array}{l|l}
\liminf _{j \rightarrow \infty} \int_{\Omega} f\left(\nabla u_{j}\right) \mathrm{d} x & \begin{array}{l}
\left(u_{j}\right) \subset W^{1, r}\left(\Omega, \mathbb{R}^{N}\right) \\
u_{j} \rightarrow u \text { in } W^{1, q}\left(\Omega, \mathbb{R}^{N}\right)
\end{array}
\end{array}\right\} .
$$


Key results concerning properties of such functionals may be found in work by Bouchitté, Fonseca and Malý (see $[15,24]$ - in fact they consider more general integrands of the form $f=f(x, u, \nabla u)$ ). Equipped with this definition, we may consider the relaxed problem

$$
\bar{m}:=\inf \left\{\mathscr{F}^{r, q}(v, \Omega): v \in W^{1, q}\left(\Omega ; \mathbb{R}^{N}\right), v=g \text { on } \partial \Omega\right\} .
$$

Now suppose that $f$ is a quasiconvex integrand satisfying the "non-standard" growth condition

$$
c_{0}|\xi|^{q}-c_{1} \leq f(\xi) \leq c_{2}(1+|\xi|)^{r}
$$

for $1<q<r<\infty$. If, as discussed above, we have established that the variational integral $F(\cdot, \Omega)$ is sequentially weakly lower semicontinuous when the sequence $\left(u_{j}\right) \subset W^{1, r}\left(\Omega ; \mathbb{R}^{N}\right)$ converges to $u \in W^{1, r}\left(\Omega ; \mathbb{R}^{N}\right)$ weakly in $W^{1, q}\left(\Omega ; \mathbb{R}^{N}\right)$, it follows that $\mathscr{F}^{r, q}(\cdot, \Omega)$ agrees with $F(\cdot, \Omega)$ on $W^{1, r}\left(\Omega ; \mathbb{R}^{N}\right)$, and we may say that it is indeed an extension of the original variational integral.

In addition, since $f$ is $q$-coercive, it can straightforwardly be shown that $\mathscr{F}^{r, q}(\cdot, \Omega)$ is lower semicontinuous in the sequential weak topology of $W^{1, q}\left(\Omega ; \mathbb{R}^{N}\right)$. Hence a minimising sequence $\left(u_{j}\right) \subset W^{1, q}\left(\Omega ; \mathbb{R}^{N}\right)$ approximating $\bar{m}$ in (1.7) (that is, satisfying $\mathscr{F}^{r, q}\left(u_{j}, \Omega\right) \rightarrow \bar{m}$ ) converges weakly (taking a subsequence if necessary) to a limit map $u \in W^{1, q}\left(\Omega ; \mathbb{R}^{N}\right)$, which thus satisfies

$$
\mathscr{F}^{r, q}(u, \Omega)=\bar{m},
$$

meaning that $u$ is a solution to the relaxed problem (1.7). Regularity results for quasiconvex integrands satisfying such non-standard growth conditions may be found in recent work by Schmidt $[44,45]$.

\subsection{Lower semicontinuity and relaxation in BV}

Let us consider again the coercivity property (1.6): when $q=1$, it is very hard to prove that a minimising sequence of $F$ is relatively compact in the weak topology of $W^{1,1}\left(\Omega ; \mathbb{R}^{N}\right)$. Therefore in this case it is useful to prove lower semicontinuity without assuming that maps $\nabla u_{j}$ converge weakly in $L^{1}\left(\Omega ; \mathbb{R}^{N}\right)$ to $\nabla u$. This was done by Dal Maso in the scalar $(N=1)$ case [19]; in the vector-valued case for $f$ convex, results have been obtained, for example, by Reshetnyak [41], Ball and Murat [14], and Aviles and Giga [13]. For the quasiconvex case, a first result in this direction was obtained by Fonseca in [23], who proved that if $f$ is quasiconvex and satisfies linear growth conditions - i.e. (1.2) for $r=1$, then lower semicontinuity obtains for a sequence $\left(u_{j}\right) \subset W^{1,1}\left(\Omega ; \mathbb{R}^{N}\right)$ converging strongly in $L^{1}\left(\Omega ; \mathbb{R}^{N}\right)$ to $u \in W^{1,1}\left(\Omega ; \mathbb{R}^{N}\right)$, provided the $\left(u_{j}\right)$ are also bounded in $W^{1,1}\left(\Omega ; \mathbb{R}^{N}\right)$. Subsequently, the hypothesis of boundedness in $W^{1,1}\left(\Omega ; \mathbb{R}^{N}\right)$ was removed by Fonseca and Muller in [26].

Nevertheless, such results these are not satisfactory for most applications, since most existence theorems for functionals with linear coercivity conditions involve the space $\operatorname{BV}\left(\Omega ; \mathbb{R}^{N}\right)$, of functions $u \in L^{1}\left(\Omega ; \mathbb{R}^{N}\right)$ whose distributional derivative can be represented by a matrix-valued Radon measure in $\Omega$. The main reason for this is because it has better compactness properties. Hence, as mentioned above, we introduce the Lebesgue-Serrin Extension $\mathscr{F}_{\text {loc }}$ as defined in (1.4): the properties of such a functional in the case where $f$ is quasiconvex, has linear growth, and $r=1$ have been studied extensively by Ambrosio and Dal Maso [8], and Fonseca and Müller [27] (in the latter, the case of general integrands $f=f(x, u, \nabla u)$ of linear growth is treated). Most notably they prove that for every open set $\Omega \subset \mathbb{R}^{n}$ and every $u \in \operatorname{BV}\left(\Omega ; \mathbb{R}^{N}\right)$ we have

$$
\mathscr{F}_{\text {loc }}(u, \Omega)=\int_{\Omega} f(\nabla u(x)) \mathrm{d} x+\int_{\Omega} f^{\infty}\left(\frac{D^{s} u}{\left|D^{s} u\right|}\right)\left|D^{s} u\right| .
$$

This result provided one of the main motivations for the lower semicontinuity result in this paper. In this connection see also Rindler [42] for a proof that avoids the use of Alberti's rank-one theorem. This integral representation in the convex case was proved earlier by Goffman and Serrin in [29]. Other related material appears in work by Aviles and Giga [13], Ambrosio et al. [11], Ambrosio and Pallara [12], and Fonseca and Rybka [28]. 


\subsection{Superlinear growth conditions in the BV setting}

The majority of previous results concerning lower semicontinuity in BV of quasiconvex integrals concern integrals $f$ that satisfy linear growth conditions. Let us now turn our attention to superlinear growth conditions: in [30], Kristensen shows that when $f$ is quasiconvex and satisfies the growth condition (1.2) for $r \in\left[1, \frac{n}{n-1}\right)$, $\mathscr{F}_{\text {loc }}$ satisfies the lower bound

$$
\mathscr{F}_{\text {loc }}(u, \Omega) \geq \int_{\Omega} f(\nabla u) \mathrm{d} x
$$

whenever $u \in \operatorname{BV}\left(\Omega ; \mathbb{R}^{N}\right)$, where again $\nabla u$ is the density of the absolutely continuous part of the measure $D u$ with respect to Lebesgue measure. The final result presented here may be seen as an extension of this work: indeed some elements of the proof come directly from Kristensen's paper. In [48], a lower semicontinuity result in the sequential weak* topology of BV is obtained for $1<r<2$. Hence, for $n>2$, we can take $r>\frac{n}{n-1}$. This result requires us to assume additionally that the maps $\left(u_{j}\right)$ are bounded uniformly in $L_{\text {loc }}^{q}$ for $q$ suitably large, as well as, for technical reasons, an additional regularity requirement on the limit map $u$.

\section{FunCtions of BOUnded VARIATION}

Here we shall provide a brief overview of some properties of the space of functions of Bounded Variation that are key in the context of this paper. For a thorough treatment of this space, we refer to the monograph of Ambrosio, Fusco and Pallara [10].

\subsection{Basic properties and weak* compactness}

Let $\Omega$ be a generic open set in $\mathbb{R}^{n}$. Recall that a function $u$ is said to be in $\operatorname{BV}\left(\Omega ; \mathbb{R}^{N}\right)$ if it is in $L^{1}\left(\Omega ; \mathbb{R}^{N}\right)$ and its distributional derivative can be represented by a matrix-valued Radon measure $D u=\left(D_{i} u^{j}\right)_{1 \leq j \leq n}^{1 \leq j \leq N}$ in $\Omega$, where $D_{i} u^{j}$ are signed Radon measures on $\Omega$.

Now recall that if $u,\left(u_{j}\right) \subset \operatorname{BV}\left(\Omega ; \mathbb{R}^{N}\right)$, then we say that $\left(u_{j}\right)$ weakly* converges to $u$ in $\operatorname{BV}\left(\Omega ; \mathbb{R}^{N}\right)$ if $u_{j} \rightarrow u$ strongly in $L^{1}\left(\Omega ; \mathbb{R}^{N}\right)$, and $D u_{j}$ converges weakly* to $D u$ in $\mathscr{M}\left(\Omega ; \mathbb{R}^{N \times n}\right)$, where $\mathscr{M}\left(\Omega ; \mathbb{R}^{N \times n}\right)$ is the space of $N \times n$ matrix-valued Borel measures on $\Omega$. Since the space of signed Radon measures on $\Omega$ is isometrically isomorphic to the dual space of the $\|\cdot\|_{\infty}$-closure of compactly-supported continuous functions on $\Omega,\left[C_{0}\left(\Omega ; \mathbb{R}^{N \times n}\right)\right]^{*}$, this means

$$
\lim _{j \rightarrow \infty} \int_{\Omega} \phi \mathrm{d} D u_{j}=\int_{\Omega} \phi \mathrm{d} D u \quad \forall \phi \in C_{0}(\Omega) .
$$

Now we state the following compactness theorem for functions in BV. Since the Sobolev Space $W^{1,1}$ has no similar compactness property, this gives us good justification for the introduction of BV in the Calculus of Variations.

Theorem 2.1. Let $\left(u_{j}\right)$ be a sequence in $B V\left(\Omega ; \mathbb{R}^{N}\right)$ satisfying

$$
\sup \left\{\int_{A}\left|u_{j}\right| \mathrm{d} x+\left|D u_{j}\right|(A): j \in \mathbb{N}\right\}<\infty \quad \forall A \subset \subset \Omega \text { open. }
$$

Then there is a subsequence $\left(u_{j_{k}}\right)$ converging in $L^{1}\left(\Omega ; \mathbb{R}^{N}\right)$ to $u \in B V\left(\Omega ; \mathbb{R}^{N}\right)$. If $\Omega$ has a compact Lipschitz boundary and $\left(u_{j}\right)$ is bounded in $B V\left(\Omega ; \mathbb{R}^{N}\right)$, then the subsequence converges weakly* in $B V$ to $u$.

We remark that we may generalise the last sentence of the theorem, requiring only that $\Omega$ is a bounded extension domain. This means that exists a linear and continuous extension operator that extends BV functions defined on $\Omega$ into $\mathbb{R}^{n}$ in a suitably "good" way (for further details, refer to [10]). 


\subsection{Mollification of BV functions}

Let $B$ denote the open unit ball in $\mathbb{R}^{n}$ and write $B_{\epsilon}$ to mean the ball of radius $\epsilon>0$ centred at the origin. Let $\phi$ be a symmetric convolution kernel in $\mathbb{R}^{n}$. That is, it satisfies $\phi \in C_{c}^{\infty}(B), \phi \geq 0, \int \phi=1, \phi(x)=\phi(-x)$, and $\operatorname{supp}(\phi) \subset \subset B$. Now let $\left(\phi_{\epsilon}\right)_{\epsilon>0}$ denote the family of mollifiers $\phi_{\epsilon}(x)=\epsilon^{-n} \phi(x / \epsilon)$. Given $\mu$, a vector-valued Radon measure on $\Omega$, define the function

$$
\mu * \phi_{\epsilon}(x):=\int_{\Omega} \phi_{\epsilon}(x-y) \mathrm{d} \mu(y)=\epsilon^{-n} \int_{\Omega} \phi\left(\frac{x-y}{\epsilon}\right) \mathrm{d} \mu(y)
$$

whenever $x \in \Omega_{\epsilon}:=\{x \in \Omega: \operatorname{dist}(x, \partial \Omega)>\epsilon\}$.

It is often useful to approximate BV functions with smooth functions using mollification, for example in the proof of Lemma 3.3. The following proposition states some key properties in this context.

Proposition 2.2. Let $u \in B V\left(\Omega ; \mathbb{R}^{N}\right)$ and let $\left(\phi_{\epsilon}\right)_{\epsilon>0}$ be a family of mollifiers.

(a) The following identity holds in $\Omega_{\epsilon}$

$$
\nabla\left(u * \phi_{\epsilon}\right)=D u * \phi_{\epsilon}
$$

(b) If $U \subset \subset \Omega$ is such that $|D u|(\partial U)=0$, then

$$
\lim _{\epsilon \searrow 0}\left|D\left(u * \phi_{\epsilon}\right)\right|(U)=|D u|(U) .
$$

(c) If $K \subset \Omega$ is a compact set, then for all $\epsilon \in(0, \operatorname{dist}(K, \partial \Omega))$

$$
\int_{K}\left|u * \phi_{\epsilon}-u\right| \mathrm{d} x \leq \epsilon|D u|(\Omega) .
$$

\subsection{Decomposition of derivative}

Using the Radon-Nikodým Theorem, for a given BV function $u$ we may decompose $D u$ as $D u=D^{a} u+D^{s} u$, where $D^{a} u$ is the absolutely continuous part of $D u$ with respect to the Lebesgue measure $\mathscr{L}^{n}$ and $D^{s} u$ is the singular part of $D u$ with respect to $\mathscr{L}^{n}$. By the Besicovitch Derivation Theorem, we may write $D^{a} u=\nabla u\left\lfloor\mathscr{L}^{n}\right.$, where $\nabla u$ is the unique $L^{1}$ function given by

$$
\nabla u(x)=\lim _{\varrho \rightarrow 0} \frac{D u(B(x, \varrho))}{\mathscr{L}^{n}(B(x, \varrho))}
$$

at all points $x \in \Omega$ where this limit is finite. In fact, we do not need to take balls of radius $\varrho$ in the above expression: if we have any bounded, convex, open set $C$ containing the origin, and write $C(x, \varrho):=x+\varrho C$, then we also obtain the same limit when we replace all instances of $B(x, \varrho)$ with $C(x, \varrho)$. A proof of this may be found, for instance, in [8]. By a result of Calderón and Zygmund, for any function $u \in \operatorname{BV}\left(\Omega ; \mathbb{R}^{N}\right)$ this expression is finite at $\mathscr{L}^{n}$-almost every point of $\Omega$.

Now recall that $u$ is said to be approximately continuous at a point $x \in \Omega$ if

$$
\lim _{\varrho \searrow 0} f_{B(x, \varrho)}|u(y)-z| \mathrm{d} y=0
$$

for some $z \in \mathbb{R}^{N}$ (which will be unique for each $x$ ). The set of points in $\Omega$ where this property does not hold is called the approximate discontinuity set and denoted $S_{u}$. Now we specify, among these approximate discontinuity points, those that correspond to an approximate jump discontinuity between two values along a direction $\nu$. To do this we introduce the notation

$$
\left\{\begin{array}{l}
B_{\varrho}^{+}(x, \nu):=\{y \in B(x, \varrho):\langle y-x, \nu\rangle>0\} \\
B_{\varrho}^{-}(x, \nu):=\{y \in B(x, \varrho):\langle y-x, \nu\rangle<0\}
\end{array}\right.
$$


to denote the two half balls contained in $B(x, \varrho)$ split by the hyperplane that passes through $x$ and is orthogonal to $\nu$. Let $u \in L_{\text {loc }}^{1}\left(\Omega ; \mathbb{R}^{N}\right)$ and $x \in \Omega$. Then $x$ is an approximate jump point of $u$ if there exist $a, b \in \mathbb{R}^{N}$ and $\nu \in \mathbb{S}^{n-1}$ (sphere of radius 1 in $\mathbb{R}^{n}$ ) such that $a \neq b$ and

$$
\lim _{\varrho \searrow 0} f_{B_{\varrho}^{+}(x, \nu)}|u(y)-a| \mathrm{d} y=0, \quad \lim _{\varrho \searrow 0} f_{B_{\varrho}^{-}(x, \nu)}|u(y)-b| \mathrm{d} y=0 .
$$

The triplet $(a, b, \nu)$, uniquely determined by (2.1) up to a permutation of $(a, b)$ and a change of sign of $\nu$, is denoted by $\left(u^{+}(x), u^{-}(x), \nu_{u}(x)\right)$. The set of approximate jump points of $u$ is denoted $J_{u}$. It can be shown that $J_{u}$ is a Borel subset of $S_{u}$ and that there exist Borel functions

$$
\left(u^{+}(x), u^{-}(x), \nu_{u}(x)\right): J_{u} \rightarrow \mathbb{R}^{N} \times \mathbb{R}^{N} \times \mathbb{S}^{n-1}
$$

such that $(2.1)$ is satisfied at any $x \in J_{u}$. In fact, for $u \in \operatorname{BV}\left(\Omega ; \mathbb{R}^{N}\right), S_{u}$ is a countably $\mathscr{H}^{n-1}$-rectifiable set and if we fix an orientation $\bar{\nu}$ of $S_{u}$, we have $\bar{\nu}=\nu_{u}(x)$ for all $x \in J_{u}$. This allows us to give a characterisation of $D u$ at all points $x$ of $J_{u}$, namely that it can be computed by difference of the one-sided limits $u^{+}(x)$ and $u^{-}(x)$ of $u$ on either side of the jump set $J_{u}$ along the normal vector $\nu_{u}(x)$. More precisely, we have the following result, attributable to Federer and Vol'pert.

Theorem 2.3. Let $u \in B V\left(\Omega ; \mathbb{R}^{N}\right)$. Then $S_{u}$ is countably $\mathscr{H}^{n-1}$-rectifiable and $\mathscr{H}^{n-1}\left(S_{u} \backslash J_{u}\right)=0$. Moreover, we have

$$
D u\left\lfloor J_{u}=\left(u^{+}-u^{-}\right) \otimes \nu_{u} \mathscr{H}^{n-1}\left\lfloor J_{u}\right.\right.
$$

In addition, for $u \in \operatorname{BV}\left(\Omega ; \mathbb{R}^{N}\right)$, there is a countable sequence of $C^{1}$ hypersurfaces $\Gamma_{i}$, say, which covers $\mathscr{H}^{n-1}$ almost all of $S_{u}$, i.e.

$$
\mathscr{H}^{n-1}\left(S_{u} \backslash \bigcup_{i=1}^{\infty} \Gamma_{i}\right)=0 .
$$

\subsection{Decomposition of $D^{s} u$ and rank-one properties}

By these above definitions and results, we are now in a position to further split $D^{s} u$ into two parts: for any $u \in \operatorname{BV}\left(\Omega ; \mathbb{R}^{N}\right)$, the measures

$$
D^{j} u:=D^{s} u\left\lfloor J_{u}, \quad D^{c} u:=D^{s} u\left\lfloor\left(\Omega \backslash S_{u}\right)\right.\right.
$$

are called respectively the jump part of the derivative and the Cantor part of the derivative. Hence we may now decompose $D u$ as $D u=D^{a} u+D^{j} u+D^{c} u$. Notice that the above considerations about $D^{a} u$ and Theorem 2.3 imply

$$
\begin{aligned}
D^{j} u(B) & =\int_{B \cap J_{u}}\left(u^{+}(x)-u^{-}(x)\right) \otimes \nu_{u}(x) \mathrm{d} \mathscr{H}^{n-1}(x) \\
\text { and } & \\
D^{a} u(B) & =\int_{B} \nabla u(x) \mathrm{d} \mathscr{L}^{n}(x)
\end{aligned}
$$

for all Borel subsets $B$ of $\Omega$; for $\left|D^{j} u\right|$ and $\left|D^{a} u\right|$ we simply take the modulus of the integrands. The Lebesgue Differentiation Theorem implies that these two components of $D u$ can be obtained by restrictions of $D u$ to the points $x \in \Omega$ where $\varrho \mapsto|D u|(B(x, \varrho))$ is comparable with $\varrho^{n}$ (for $D^{a} u$ ) and $\varrho^{n-1}$ (for $D^{j} u$ ). The Cantor part of $D u$ has intermediate behaviour and is trickier to characterise: unlike the absolutely continuous and jump parts of BV functions, the Cantor parts can only be seen as a measure and cannot be recovered by classical analysis of the pointwise behaviour of a functions. Indeed the Cantor-Vitali function whose distributional derivative has no jump part and no absolutely continuous part, demonstrates that for general BV functions $u$, not all of 
$D^{s} u$ may be captured by $D^{j} u$. However, it is not too complicated to show that $D^{c} u$ vanishes on sets which are $\sigma$-finite with respect to $\mathscr{H}^{n-1}$. We define the proper subspace of $\operatorname{BV}\left(\Omega ; \mathbb{R}^{N}\right)$, called functions of Special Bounded Variation, $\operatorname{SBV}\left(\Omega ; \mathbb{R}^{N}\right)$, to be the space of BV functions where $D^{s} u=D^{j} u$ only. For an introduction to this space, we refer to $[5,6,20]$.

Let us now turn our attention to the quantity $\xi=\frac{D u}{|D u|}$ i.e. the Radon-Nikodým derivative of the measure $D u$ with respect to its variation $|D u|$ given by the expression

$$
\xi(x)=\frac{D u}{|D u|}(x)=\lim _{\varrho \rightarrow 0} \frac{D u(B(x, \varrho))}{|D u|(B(x, \varrho))}, x \in \Omega .
$$

We consider $\xi(x)$ at the various parts of $\Omega$ that are seen by these various components of $D u$. It follows straightforwardly from the basic properties described above that for $\left|D^{a} u\right|$-almost all $x \in \Omega$ we have

$$
\xi(x)=\frac{\nabla u(x)}{|\nabla u(x)|},
$$

and for $\mathscr{H}^{n-1}$-almost all $x \in J_{u}$,

$$
\xi(x)=\frac{u^{+}(x)-u^{-}(x)}{\left|u^{+}(x)-u^{-}(x)\right|} \otimes \nu_{u}(x) .
$$

Note that in this case, $\xi(x)$ is a rank-one matrix. It is much harder to establish properties of $\xi(x)$ for points $x$ that are seen by the measure $D^{c} u$. In [2], Alberti proved the famous result that $\xi(x)$ is also rank-one for $\left|D^{c} u\right|$-almost every point. The proof of this property is very long and involved; a simpler proof based on the area formula and Reshetnyak continuity theorem is given in [3], but this proof only works for monotone BV functions. These properties of $\xi(x)$ are instrumental in the proof of Theorem 4.1, in particular in the context of the key blow-up lemma that the last part of this section is devoted to.

\subsection{Sets of finite perimeter}

Let $E$ be a subset of $\Omega$, and define the characteristic function $\mathbf{1}_{E}$ of $E$ as

$$
\mathbf{1}_{E}(x)= \begin{cases}1 & \text { if } x \in E \\ 0 & \text { if } x \in \Omega \backslash E .\end{cases}
$$

We say that a set $E$ is of finite perimeter in $\Omega$ if $\mathbf{1}_{E} \in \operatorname{BV}\left(\Omega ; \mathbb{R}^{N}\right)$. Now define the reduced boundary of $E$, $\left(\partial^{*} E \cap \Omega\right)$, as

$$
\left(\partial^{*} E \cap \Omega\right)=S_{\mathbf{1}_{E}} .
$$

Note that $\left|D \mathbf{1}_{E}\right|(\Omega)=\mathscr{H}^{n-1}\left(\partial^{*} E \cap \Omega\right)$ for every $E$ of finite perimeter. It is easy to verify that this notion of perimeter coincides with the elementary one, particularly when $E$ is a polyhedron. In [21], De Giorgi shows that if $E$ is a set of finite perimeter, then there exists a sequence of polyhedra $\left(P_{j}\right)$ such that $\left|\left(\left(P_{j} \backslash E\right) \cup\left(E \backslash P_{j}\right)\right) \cap \Omega\right| \rightarrow 0$, and

$$
\mathscr{H}^{n-1}\left(\partial^{*}\{u>t\}\right)=\lim _{j \rightarrow \infty} \mathscr{H}^{n-1}\left(\partial P_{j} \cap \Omega\right) .
$$

This shows that the measure-theoretic notion of perimeter is a sensible extension of the elementary one.

\subsection{Properties of blow-up limits on the singular part}

In this section we state a result from [8] that is essential to our proof of Theorem 4.1. First let us note that when blowing up a function $u \in \operatorname{BV}\left(\Omega ; \mathbb{R}^{N}\right)$ at a point $x_{0} \in \Omega$, we will need to use the following identities. Let $Q$ denote the open unit cube $\left(-\frac{1}{2}, \frac{1}{2}\right)^{n}$ in $\mathbb{R}^{n}$ and, consistent with the previous section, define

$$
Q\left(x_{0}, \varrho\right):=\left\{\varrho y+x_{0}: y \in Q\right\} .
$$


Now for $y \in Q$ and $\varrho$ sufficiently small, let

$$
u_{\varrho}(y):=\varrho^{-1} u\left(x_{0}+\varrho y\right) .
$$

It follows from basic definitions that

$$
D u_{\varrho}(Q)=\varrho^{-n} D u\left(Q\left(x_{0}, \varrho\right)\right), \quad \text { and } \quad\left|D u_{\varrho}\right|(Q)=\varrho^{-n}|D u|\left(Q\left(x_{0}, \varrho\right)\right) .
$$

Moreover, recall that the support of a measure $\mu$ in $\Omega$ is defined by

$$
\operatorname{supp}(\mu)=\{x \in \Omega: \mu(\Omega \cap B(x, \varrho))>0 \forall \varrho>0\} .
$$

Theorem 2.4. Let $u \in B V\left(\Omega ; \mathbb{R}^{N}\right)$, and let $\xi: \Omega \rightarrow \mathbb{R}^{N \times n}$ denote the density of Du with respect to $|D u|$. Then, for $\left|D^{s} u\right|$-almost all $x_{0} \in \Omega$ we have $\left|\xi\left(x_{0}\right)\right|=1, \operatorname{rank}\left(\xi\left(x_{0}\right)\right)=1$, and

$$
\lim _{\varrho \rightarrow 0^{+}} \frac{D u\left(Q\left(x_{0}, \varrho\right)\right)}{|D u|\left(Q\left(x_{0}, \varrho\right)\right)}=\xi\left(x_{0}\right), \quad \lim _{\varrho \rightarrow 0^{+}} \frac{D u\left(Q\left(x_{0}, \varrho\right)\right)}{\varrho^{n}}=+\infty .
$$

Let $x \in \operatorname{supp}(|D u|)$ with these properties, and write $\xi\left(x_{0}\right)=\eta \otimes \nu$ where $\eta \in \mathbb{R}^{n}, \nu \in \mathbb{R}^{N},|\eta|=|\nu|=1$. Now let

$$
v_{\varrho}(y)=\frac{\varrho^{n}}{|D u|\left(Q\left(x_{0}, \varrho\right)\right)}\left(u_{\varrho}(y)-m_{\varrho}\right),
$$

where $u_{\varrho}$ is defined in (2.2) and $m_{\varrho}$ is the mean value of $u_{\varrho}$ on $Q$ (with respect to Lebesgue measure). Then for $\varrho$ sufficiently small and for every $0<\sigma \leq 1$ we have

$$
\int_{Q} v_{\varrho} \mathrm{d} y=0, \quad\left|D v_{\varrho}\right|(\sigma Q)=\frac{|D u|\left(Q\left(x_{0}, \sigma \varrho\right)\right)}{|D u|\left(Q\left(x_{0}, \varrho\right)\right)} \leq 1 .
$$

Moreover, for every $0<\sigma<1$ there exists a decreasing sequence $\left(\varrho_{k}\right)$ converging to 0 such that $\left(v_{\varrho_{k}}\right)$ converges weakly* in $B V\left(Q ; \mathbb{R}^{N}\right)$ to a function $v \in B V\left(Q ; \mathbb{R}^{N}\right)$ satisfying

$$
|D v|(\sigma \bar{Q}) \geq \sigma^{n}
$$

and which can be represented as

$$
v(y)=\psi(\langle y, \nu\rangle) \eta
$$

for a suitable non-decreasing function $\psi:(a, b) \rightarrow \mathbb{R}$, where

$$
a=\inf \{\langle y, \nu\rangle: y \in \mathbb{R}\}, \quad b=\sup \{\langle y, \nu\rangle: y \in \mathbb{R}\} .
$$

In this connection see also Larsen [31], where a similar result is obtained that allows one to even assume that $|D v|(Q)=1$ and $|D v|(\partial Q)$ for the blow-up limit.

We will now make one remark on the proof of this theorem, which is important in the context of the proof of Proposition 4.10. In [8], it is shown that we have

$$
\limsup _{\varrho \rightarrow 0^{+}} \frac{|D u|\left(Q\left(x_{0}, \sigma \varrho\right)\right)}{|D u|\left(Q\left(x_{0}, \varrho\right)\right)}>\sigma^{n} .
$$

If this were false, then there would exist $\varrho_{0}>0$ such that

$$
|D u|\left(Q\left(x_{0}, \sigma \varrho\right)\right) \leq \sigma^{n}|D u|\left(Q\left(x_{0}, \varrho\right)\right)
$$


for all $0<\varrho \leq \varrho_{0}$. This implies that for any $j \in \mathbb{N},|D u|\left(Q\left(x_{0}, \sigma^{j} \varrho_{0}\right)\right) \leq \sigma^{j n}|D u|\left(Q\left(x_{0}, \varrho_{0}\right)\right)$. Hence we obtain

$$
\begin{aligned}
|D u|\left(Q\left(x_{0}, \varrho_{0}\right)\right) & \geq \frac{|D u|\left(Q\left(x_{0}, \sigma^{j} \varrho_{0}\right)\right)}{\sigma^{j n}} \\
& \rightarrow \infty \quad \text { as } j \rightarrow \infty,
\end{aligned}
$$

which is a contradiction. Hence the decreasing seqeunce $\left(\varrho_{k}\right)$ in this result may be chosen to satisfy

$$
\lim _{k \rightarrow \infty}\left|D v_{\varrho_{k}}\right|(\sigma Q) \geq \sigma^{n} .
$$

Now note that if $C \subset[0, \infty)$ is a countable set, then we may also obtain the property in (2.8) by imposing the additional restriction $\varrho \notin C$. We do this by choosing a suitable $\varrho_{0}$ such that $\sigma^{j} \varrho_{0} \notin C$ for all $j$ (this must hold as $C$ is countable).

\section{UPPER BOUND IN SUBQUADRATIC GROWTH CASE}

In this section we obtain an upper bound for the Lebesgue-Serrin extension $\mathscr{F}_{\text {loc }}$ as defined in (1.4), where $f$ is continuous and satisfies the growth condition (1.2) for $r \in[1,2)$. Hence, for $n>2, f$ may have larger growth than in the rest of this paper. We shall assume additionally that $f$ has linear growth in certain rank-one directions. That is,

$$
0 \leq f(\xi) \leq C(|\xi|+1)
$$

whenever

$$
\xi=\eta \otimes \nu, \quad \eta \in \operatorname{span}\{u(y): y \in \Omega\}, \nu \in \mathbb{R}^{n} .
$$

The results proved here are still interesting if we assume $f$ has linear growth on all rank-one matrices. However in the context of the lower semicontinuity result proved in the next section, where $f$ is additionally assumed to be quasiconvex, we shall show that this is too strong an assumption.

First let us collect some elemenary facts for functionals such as $\mathscr{F}_{\text {loc }}$. A key reference for general properties is [17]. For every $z \in \mathbb{R}^{n}$ define the translation operator $T_{z}$ by $\left(T_{z} u\right)(x)=u(x-z)$ and $T_{z} \Omega=\left\{x \in \mathbb{R}^{n}\right.$ : $x-z \in \Omega\}=z+\Omega$. For every $\varrho>0$, define the homothety operator $\theta_{\varrho}$ by $\left(\theta_{\varrho} u\right)(x)=(1 / \varrho)(u(\varrho x))$ and $\theta_{\varrho} \Omega=\left\{x \in \mathbb{R}^{n}: \varrho x \in \Omega\right\}=(1 / \varrho) \Omega$. The following proposition states some important facts about $\mathscr{F}$ (as defined in (1.3)) and $\mathscr{F}_{\text {loc }}$ that come directly from their definitions. Note that no restriction on the exponent $r$ is required here.

Proposition 3.1. Let $u \in B V\left(\Omega ; \mathbb{R}^{N}\right)$. Let $f: \mathbb{R}^{N \times n} \rightarrow \mathbb{R}$ be a continuous function satisfying the growth condition (1.2) for some exponent $1 \leq r<\infty$. Then the corresponding Lebesgue-Serrin extension $\mathscr{F}$ defined for this $f$ satisfies the following properties:

(a) $\mathscr{F}\left(T_{z} u, T_{z} \Omega\right)=\mathscr{F}(u, \Omega)$ for every $z \in \mathbb{R}^{n}$,

(b) $\mathscr{F}(u+\eta, \Omega)=\mathscr{F}(u, \Omega)$ for every $\eta \in \mathbb{R}^{N}$,

(c) $\mathscr{F}\left(\theta_{\varrho} u, \theta_{\varrho} \Omega\right)=\varrho^{-n} \mathscr{F}(u, \Omega)$ for every $\varrho>0$.

Identical statements hold for $\mathscr{F}_{\mathrm{loc}}$.

The next proposition shows that, provided we assume that $f$ is coercive, then by a straightforward diagonalisation argument and compactness properties in BV we have that $\mathscr{F}$ and $\mathscr{F}$ loc are attained and are lower semicontinuous in the weak* topology of BV.

Proposition 3.2. Let $f$ be as in Proposition 3.1. Assume in addition that $f$ satisfies, for some constant $c_{0}>0$,

$$
f(\xi) \geq c_{0}|\xi|
$$

for all $\xi \in \mathbb{R}^{N \times n}$. Then 
(a) If $\mathscr{F}(u, \Omega)<\infty$, then the value is attained. That is, there exists a sequence $\left(u_{j}\right)$ in $W^{1, r}\left(\Omega ; \mathbb{R}^{N}\right)$ such that $u_{j} \stackrel{*}{\rightarrow} u$ in $B V\left(\Omega ; \mathbb{R}^{N}\right)$ and

$$
\lim _{j \rightarrow \infty} \int_{\Omega} f(\nabla u) \mathrm{d} x=\mathscr{F}(u, \Omega) .
$$

(b) If $\left(u_{j}\right)$ is a sequence in $B V\left(\Omega ; \mathbb{R}^{N}\right)$ converging weakly* in $B V$ to $u \in B V\left(\Omega ; \mathbb{R}^{N}\right)$, and $\mathscr{F}\left(u_{j}, \Omega\right)<\infty$ for all $j$, then

$$
\liminf _{j \rightarrow \infty} \mathscr{F}\left(u_{j}, \Omega\right) \geq \mathscr{F}(u, \Omega)
$$

Identical statements hold for $\mathscr{F}_{\text {loc }}$.

\subsection{Upper bound for certain functions in SBV}

We first establish an upper bound for $\mathscr{F}_{\text {loc }}(u, \Omega)$ for specific types of functions $u$ in SBV, namely those that are constant almost everywhere (and hence have absolutely continuous part zero), whose jump set is the union of finitely many polyhedra.

Lemma 3.3. Let $\Omega$ be a bounded, open extension domain in $\mathbb{R}^{n}$. Suppose $u \in S B V\left(\Omega ; \mathbb{R}^{N}\right)$ is such that

$$
|\nabla u(x)|=0
$$

for $\mathscr{L}^{n}$-almost all $x \in \Omega$, and that the set $J_{u}$ of approximate jump points of $u$ is the union of finitely many polyhedra. Let $f: \mathbb{R}^{N \times n} \rightarrow \mathbb{R}$ be a continuous function satisfying the growth condition (1.2) for some exponent $1 \leq r<2$. Assume also that it has linear growth on matrices $\xi$ satisfying (3.1). Then

$$
\mathscr{F}_{\text {loc }}(u, \Omega) \leq C\left(\mathscr{L}^{n}(\Omega)+\left|D^{s} u\right|(\Omega)\right)
$$

for some constant $C>0$ dependent on $f$.

Proof of Lemma 3.3. We argue by mollification. Let $\left(\phi_{\epsilon}\right)_{\epsilon>0}$ be family of mollifiers, i.e. $\phi_{\epsilon}(x)=\epsilon^{-n} \phi(x / \epsilon)$, where $\phi$ is a symmetric convolution kernel in $\mathbb{R}^{n}$ (so it satisfies $\phi \in C_{c}^{\infty}(B(0,1)), \phi \geq 0, \int \phi=1, \phi(x)=\phi(-x)$, and $\operatorname{supp}(\phi) \subset \subset B(0,1))$. We wish to mollify on all of $\Omega$ : since it has a Lipschitz boundary, we can extend $u$ onto all of $\mathbb{R}^{n}$ so that

$$
|D u|\left(\mathbb{R}^{n}\right) \leq C|D u|(\Omega),
$$

and $u$ still satisfies $\nabla u=0, J_{u}$ is the union of finitely many polyhedra, and $\operatorname{span}\left\{u(y): y \in \mathbb{R}^{n}\right\}=\operatorname{span}\{u(y)$ : $y \in \Omega\}$. Now define, for $\epsilon>0, u_{\epsilon}(x):=\left(u * \phi_{\epsilon}\right), x \in \Omega$. Recall from Proposition 2.2 that we have

$$
\nabla u_{\epsilon}(x)=\left(D u * \phi_{\epsilon}\right)(x)=\epsilon^{-n} \int_{B(x, \epsilon)} \phi\left(\frac{y}{\epsilon}\right) \mathrm{d} D u(y) .
$$

Let $x \in \Omega$ and consider $B(x, \epsilon)$ : if $B(x, \epsilon) \cap J_{u}=\emptyset$, then $D u=\nabla u=0$ on $B(x, \epsilon)$, and so

$$
f\left(\nabla u_{\epsilon}(x)\right)=f(0) .
$$

If $B(x, \epsilon) \cap J_{u} \neq \emptyset$, and the intersection of this ball and the jump set is just part of the face of a single polyhedron, then, on this ball, we have

$$
D u=D^{s} u=a \otimes \nu \mathscr{H}^{n-1}\left\lfloor J_{u},\right.
$$

where $a$ is just the difference of (constant) values of $u$ on either side of the face, and $\nu$ is a unit normal to this face in the appropriate direction. Hence we have

$$
\begin{aligned}
\left|\nabla u_{\epsilon}(x)\right| & =\left|\epsilon^{-n} \int_{B(x, \epsilon) \cap J_{u}} \phi\left(\frac{y}{\epsilon}\right)(a \otimes \nu) \mathrm{d} \mathscr{H}^{n-1}(y)\right| \\
& \leq \epsilon^{-n} \mathscr{H}^{n-1}\left(J_{u} \cap B(x, \epsilon)\right)|a \otimes \nu|
\end{aligned}
$$


Recall that by the given finiteness condition on $f^{\infty}$, it follows that $f$ satisfies the linear growth condition

$$
0 \leq f(\xi) \leq C(1+|\xi|)
$$

for all matrices $\xi$ of the form $\eta \otimes \nu$, where $\nu \in \mathbb{R}^{n}$ and $\eta \in \operatorname{span}\{u(y): y \in \Omega\}$. Since $a \otimes \nu$ is of this form, we get

$$
\begin{aligned}
f\left(\nabla u_{\epsilon}\right) & \leq C\left(1+\epsilon^{-n} \mathscr{H}^{n-1}\left(J_{u} \cap B(x, \epsilon)\right)|a \otimes \nu|\right) \\
& =C\left(1+\epsilon^{-n}\left|D^{s} u\right|\left(J_{u} \cap B(x, \epsilon)\right)\right) .
\end{aligned}
$$

Note that this inequality holds even if $B(x, \epsilon) \cap J_{u}=\emptyset$. Lastly, suppose $B(x, \epsilon) \cap J_{u} \neq \emptyset$, and the intersection of this ball and the jump set contains more than just a face - i.e. it contains a corner of a polyhedron and/or multiple (albeit finitely many) polyhedra. Then we have, on this ball,

$$
D u=D^{s} u=\left(\sum_{i=1}^{m} \xi_{i}\right) \mathscr{H}^{n-1}\left\lfloor J_{u}\right.
$$

for some $m \in \mathbb{N}$, where $\xi_{i}$, similarly to above, are rank-one matrices of the form $a_{i} \otimes \nu_{i}$ corresponding to jumps $a_{i}$ along the unit normal vector $\nu_{i}$ of some face of some polyhedron in this intersection. Note that $\mathscr{H}^{n-1}\left(B(x, \epsilon) \cap J_{u}\right)$ is of order $\epsilon^{n-1}$, so

$$
\begin{aligned}
\left|\nabla u_{\epsilon}(x)\right| & =\left|\epsilon^{-n} \int_{B(x, \epsilon) \cap J_{u}} \phi\left(\frac{y}{\epsilon}\right) \mathrm{d} D^{s} u(y)\right| \\
& \leq \epsilon^{-n}\left(\sum_{i=1}^{m}\left|\xi_{i}\right|\right) \mathscr{H}^{n-1}\left(B(x, \epsilon) \cap J_{u}\right) \\
& \leq C \epsilon^{-1} \sum_{i=1}^{m}\left|\xi_{i}\right| .
\end{aligned}
$$

Now use the growth condition (1.2) on $f$ to get

$$
\begin{aligned}
f\left(\nabla u_{\epsilon}(x)\right) & \leq C\left(1+\epsilon^{-r} \sum_{i=1}^{m}\left|\xi_{i}\right|^{r}\right) \\
& \leq C(u)\left(1+\epsilon^{-r}\right),
\end{aligned}
$$

where $C(u)$ is a constant depending on $u$. Similarly to before, this inequality holds even if $B(x, \epsilon) \cap J_{u}=\emptyset$, or if this intersection only contains just a face.

Now let $\mathcal{B}$ be a maximal collection of disjoint balls of radius $\epsilon / 5$ in $\Omega$. That is, $\mathcal{B}$ is a (finite) disjoint collection of balls, and for any other ball $B^{\prime} \subset \Omega$ of radius $\epsilon / 5$,

$$
B^{\prime} \cap \bigcup_{B \in \mathcal{B}} B \neq \emptyset
$$

For $B \in \mathcal{B}$, let $R B$ denote the ball with the same centre, but of radius $\frac{R}{5} \epsilon$. Then (see, for example, [36])

$$
\Omega \subset \bigcup_{B \in \mathcal{B}} 5 B .
$$

For each $B \in \mathcal{B}$, we now consider cases as above. If $10 B \cap J_{u}=\emptyset$, then for each $x \in 5 B, B(x, \epsilon) \cap J_{u}=\emptyset$. Thus we have, from (3.3),

$$
\int_{5 B} f\left(\nabla u_{\epsilon}\right) \mathrm{d} x=|5 B| f(0)
$$


If $10 B \cap J_{u} \neq \emptyset$, and the intersection of this ball and the jump set is just the part of a face of a single polyhedron, then for each $x \in 5 B, B(x, \epsilon)$ is contained in $10 B$, and so either $B(x, \epsilon) \cap J_{u}$ is just part of a face or is empty. Hence, using (3.4),

$$
\begin{aligned}
\int_{5 B} f\left(\nabla u_{\epsilon}\right) \mathrm{d} x & \leq C|5 B|\left(1+\epsilon^{-n}\left|D^{s} u\right|\left(J_{u} \cap B(x, \epsilon)\right)\right) \\
& \leq C\left(\left(\epsilon^{n}+\left|D^{s} u\right|\left(J_{u} \cap 10 B\right)\right) .\right.
\end{aligned}
$$

Finally, if $10 B \cap J_{u} \neq \emptyset$, and the intersection of this ball and the jump set contains more than just a face, then for each $x \in 5 B, B(x, \epsilon) \cap J_{u}$ may be empty, or just part of a face, or more than just a face. Thus we use (3.5) to get

$$
\begin{aligned}
\int_{5 B} f\left(\nabla u_{\epsilon}\right) \mathrm{d} x & \leq|5 B| C(u)\left(1+\epsilon^{-r}\right) \\
& \leq C(u) \epsilon^{n-r} .
\end{aligned}
$$

Now let $\mathcal{B}_{1}, \mathcal{B}_{2}$ and $\mathcal{B}_{3}$ be the balls in $\mathcal{B}$ where (3.6), (3.7) and (3.8) hold respectively. Then $\mathcal{B}=\mathcal{B}_{1} \cup \mathcal{B}_{2} \cup \mathcal{B}_{3}$ and

$$
\sum_{B \in \mathcal{B}_{1}} \int_{5 B} f\left(\nabla u_{\epsilon}\right) \mathrm{d} x \leq C \mathscr{L}^{n}(\Omega) f(0) .
$$

Note that $\mathcal{B}_{2}$, since it only contains balls $B$ such that $10 B$ contains the polyhedral jump set of $u$ (which has Hausdorff dimension $n-1$ ), contains less than $C \epsilon^{1-n}$-many balls, where this constant depends on the jump set $J_{u}$. Hence

$$
\begin{aligned}
\sum_{B \in \mathcal{B}_{2}} \int_{5 B} f\left(\nabla u_{\epsilon}\right) \mathrm{d} x & \leq C \sum_{B \in \mathcal{B}_{2}} \epsilon^{n}+\left|D^{s} u\right|\left(J_{u} \cap 10 B\right) \\
& \leq C(u) \epsilon+C\left|D^{s} u\right|\left(J_{u} \cap \Omega\right) .
\end{aligned}
$$

Lastly, we observe that $\mathcal{B}_{3}$, since it only contains balls $B$ such that $10 B$ contains parts of the jump set that are not faces (which has Hausdorff dimension at most $n-2$ ), has cardinality of order $\epsilon^{2-n}$. Therefore

$$
\begin{aligned}
\sum_{B \in \mathcal{B}_{3}} \int_{5 B} f\left(\nabla u_{\epsilon}\right) \mathrm{d} x & \leq C(u) \sum_{B \in \mathcal{B}_{3}} \epsilon^{n-r} \\
& \leq C(u) \epsilon^{2-r}
\end{aligned}
$$

Now take a sequence $\left(\epsilon_{j}\right)$ such that $\epsilon_{j} \searrow 0$. Then $u_{\epsilon_{j}} \stackrel{*}{\rightarrow} u$ in $\operatorname{BV}\left(\Omega ; \mathbb{R}^{N}\right)$, and

$$
\begin{aligned}
\mathscr{F}_{\text {loc }}(u, \Omega) & \leq \liminf _{j \rightarrow \infty} \int_{\Omega} f\left(\nabla u_{\epsilon_{j}}\right) \mathrm{d} x \\
& \leq \liminf _{j \rightarrow \infty} \sum_{B \in \mathcal{B}} \int_{5 B} f\left(\nabla u_{\epsilon_{j}}\right) \mathrm{d} x \\
& \leq \liminf _{j \rightarrow \infty} C\left(\mathscr{L}^{n}(\Omega)+\left|D^{s} u\right|\left(J_{u} \cap \Omega\right)\right)+C(u)\left(\epsilon_{j}+\epsilon_{j}^{2-r}\right) \\
& =C\left(\mathscr{L}^{n}(\Omega)+\left|D^{s} u\right|\left(J_{u} \cap \Omega\right)\right) .
\end{aligned}
$$

This completes the proof.

Remark 3.4. Localising the proof of this result, we also obtain the upper bound

$$
\mathscr{F}_{\text {loc }}(u, U) \leq C\left(\mathscr{L}^{n}(U)+\left|D^{s} u\right|(U)\right)
$$

for any open subset $U \subset \Omega$. 


\subsection{Generalisation of result via polyhedral approximation}

We now adapt a result of Braides and Coscia [16], to obtain an upper bound for general functions $u$ in $\operatorname{BV}\left(\Omega ; \mathbb{R}^{N}\right)$.

Theorem 3.5. Let $\Omega$ be a bounded, open extension domain in $\mathbb{R}^{n}$, and $u \in B V\left(\Omega ; \mathbb{R}^{N}\right)$. Let $f: \mathbb{R}^{N \times n} \rightarrow \mathbb{R}$ be a continuous function satisfying the growth condition (1.2) for some exponent $1 \leq r<2$. Assume also that it has linear growth on matrices $\xi$ satisfying (3.1). Then

$$
\mathscr{F}_{\text {loc }}(u, \Omega) \leq C\left(\mathscr{L}^{n}(\Omega)+|D u|(\Omega)\right),
$$

where $C>0$ is a fixed constant depending on $f$.

Proof of Theorem 3.5. We shall first assume that $f$ also satisfies the coercivity condition

$$
f(\xi) \geq c_{0}|\xi|
$$

for some constant $c_{0}>0$, for all $\xi \in \mathbb{R}^{N \times n}$. Also assume that $u \in\left(C^{1} \cap \mathrm{BV}\right)\left(\Omega ; \mathbb{R}^{N}\right)$. Write $u$ in terms of its components, i.e. $u=\left(u^{(1)}, \ldots, u^{(N)}\right)$. If the dimension of $\operatorname{span}\{u(y): y \in \Omega\}$ is less than $N$, we may assume for simplicity that there exists $m<N$ such that $u^{(i)}=0$ for $i>m$ and $\operatorname{span}\{u(y): y \in \Omega\}=\operatorname{span}\left\{\epsilon_{1}, \ldots, \epsilon_{m}\right\}$, where $\left\{\epsilon_{1} \ldots, \epsilon_{N}\right\}$ is the canonical basis for $\mathbb{R}^{N}$. Otherwise we may use a change of variables. Note that the proof we give here works even if we were to assume $\operatorname{span}\{u(y): y \in \Omega\}$ has dimension $N$ and $m=N$.

Take any $i \in\{1, \ldots, m\}$ and fix $k \in \mathbb{N}$. By the co-area formula, we have

$$
\left|D u^{(i)}\right|(\Omega)=\sum_{j \in \mathbb{Z}} \int_{j / k}^{(j+1) / k} \mathscr{H}^{n-1}\left(\partial^{*}\left\{u^{(i)}>t\right\} \cap \Omega\right) \mathrm{d} t .
$$

Hence for every $j \in \mathbb{Z}$ we can find $s_{j}^{i, k} \in(j / k,(j+1) / k)$ such that

$$
\frac{1}{k} \mathscr{H}^{n-1}\left(\partial^{*}\left\{u>s_{j}^{i, k}\right\} \cap \Omega\right) \leq \int_{j / k}^{(j+1) / k} \mathscr{H}^{n-1}\left(\partial^{*}\left\{u^{(i)}>t\right\} \cap \Omega\right) \mathrm{d} t,
$$

so that

$$
\sum_{j \in \mathbb{Z}} \frac{1}{k} \mathscr{H}^{n-1}\left(\partial^{*}\left\{u>s_{j}^{i, k}\right\} \cap \Omega\right) \leq\left|D u^{(i)}\right|(\Omega) .
$$

Now take, for every $j \in \mathbb{Z}$, a polyhedron $P_{j}^{i, k}$ such that

$$
\left\{u^{(i)}>\frac{j+1}{k}\right\} \subset P_{j}^{i, k} \subset\left\{u^{(i)}>\frac{j}{k}\right\},
$$

and

$$
\mathscr{H}^{n-1}\left(\partial P_{j}^{i, k} \cap \Omega\right) \leq \mathscr{H}^{n-1}\left(\partial^{*}\left\{u>s_{j}^{i, k}\right\} \cap \Omega\right)+\frac{1}{k} 2^{-|j|} .
$$

Do this for all $i=1, \ldots m$. Now define $u_{k} \in \operatorname{SBV}\left(\Omega ; \mathbb{R}^{N}\right)$ by setting

$$
w_{k}^{(i)}(y):=\frac{j}{k} \quad \text { on } P_{j-1}^{i, k} \backslash P_{j}^{i, k},
$$

and then letting $u_{k}^{(i)}:=\max \left\{-k, \min \left\{w_{k}^{(i)}, k\right\}\right\}$. Clearly we have $\nabla u_{k}^{(i)}(x)=0$ for $\mathscr{L}^{n}$-almost all $x \in \Omega$, and there exists $j(i, k) \in \mathbb{N}$ such that

$$
J_{u_{k}^{(i)}} \cap \Omega=\bigcup_{-j(i, k) \leq j \leq j(i, k)} \partial P_{j}^{i, k}
$$


and

$$
D u_{k}^{(i)}=D^{s} u_{k}^{(i)}=\left.\sum_{j=-j(i, k)}^{j(i, k)} \frac{1}{k} \nu_{j}^{i, k} \mathscr{H}^{n-1}\right|_{\partial P_{j}^{i, k}},
$$

where $\nu_{j}^{i, k}$ is defined by

$$
D \mathbf{1}_{P_{j}^{i, k}}=\left.\nu_{j}^{i, k} \mathscr{H}^{n-1}\right|_{\partial P_{j}^{i, k}}
$$

Hence

$$
D u_{k}=D^{s} u_{k}=\left.\sum_{i=1}^{m} \sum_{j=-j(i, k)}^{j(i, k)} \frac{1}{k} \epsilon_{i} \otimes \nu_{j}^{i, k} \mathscr{H}^{n-1}\right|_{\partial P_{j}^{i, k}},
$$

and

$$
\begin{aligned}
\left|D^{s} u_{k}\right|(\Omega) & =\sum_{i=1}^{m} \sum_{j=-j(i, k)}^{j(i, k)} \frac{\left|\epsilon_{i} \otimes \nu_{j}^{i, k}\right|}{k} \mathscr{H}^{n-1}\left(\partial P_{j}^{i, k} \cap \Omega\right) \\
& =\frac{1}{k} \sum_{i=1}^{m} \sum_{j=-j(i, k)}^{j(i, k)} \mathscr{H}^{n-1}\left(\partial P_{j}^{i, k} \cap \Omega\right) \\
& \leq \frac{1}{k} \sum_{i=1}^{m} \sum_{j=-j(i, k)}^{j(i, k)}\left(\mathscr{H}^{n-1}\left(\partial^{*}\left\{u>s_{j}^{i, k}\right\} \cap \Omega\right)+\frac{1}{k} 2^{-|j|}\right) \\
& \leq \sum_{i=1}^{m}\left|D u^{(i)}\right|(\Omega)+\frac{1}{k} \\
& \leq \sqrt{m}|D u|(\Omega)+\frac{1}{k} .
\end{aligned}
$$

Since the jump set $J_{u_{k}}$ is the union of finitely many polyhedra, and by assumption $f$ has linear growth on matrices of the form $\eta \otimes \nu$ where $\eta \in \operatorname{span}\left\{\epsilon_{i}: 1 \leq i \leq m\right\}$ and $\nu \in \mathbb{R}^{n}$, we use Lemma 3.3 to get

$$
\begin{aligned}
\mathscr{F}_{\text {loc }}\left(u_{k}\right) & \leq C\left(\mathscr{L}^{n}(\Omega)+\left|D^{s} u_{k}\right|(\Omega)\right) \\
& \leq C\left(\mathscr{L}^{n}(\Omega)+|D u|(\Omega)\right)+\frac{1}{k} .
\end{aligned}
$$

Note that the sequence $\left(w_{k}\right)$ converges strongly to $u$ in $L^{\infty}\left(\Omega ; \mathbb{R}^{N}\right)$, so the truncated sequence $\left(u_{k}\right)$ converges strongly to $u$ in $L^{1}\left(\Omega ; \mathbb{R}^{N}\right)$. Moreover, the measures $\left|D u_{k}\right|$ are bounded. Hence also $u_{k} \stackrel{*}{\rightarrow} u$ in $\operatorname{BV}\left(\Omega ; \mathbb{R}^{N}\right)$, and using the lower semicontinuity of $\mathscr{F}_{\text {loc }}$ (see Prop. 3.2) we have

$$
\mathscr{F}_{\text {loc }}(u, \Omega) \leq \liminf _{k \rightarrow \infty} \mathscr{F}_{\text {loc }}\left(u_{k} \Omega\right) \leq C\left(\mathscr{L}^{n}(\Omega)+|D u|(\Omega)\right) .
$$

The result has been proved for $u \in\left(C^{1} \cap \mathrm{BV}\right)\left(\Omega ; \mathbb{R}^{N}\right)$. For general $u \in \mathrm{BV}\left(\Omega ; \mathbb{R}^{N}\right)$, it suffices to recall that by convolution and using a partition of unity (see, for example, [49]), there exists a sequence $\left(v_{k}\right) \subset$ $\left(C^{\infty} \cap \mathrm{BV}\right)\left(\Omega ; \mathbb{R}^{N}\right)$ such that $v_{k} \stackrel{*}{\rightarrow} u$ in $\operatorname{BV}\left(\Omega ; \mathbb{R}^{N}\right)$. Moreover, clearly

$$
\operatorname{span}\left\{v_{k}(y): y \in \Omega\right\}=\operatorname{span}\{u(y): y \in \Omega\},
$$

so using the result for $\left(v_{k}\right)$ and again the lower semicontinuity of $\mathscr{F}_{\text {loc }}$, we get

$$
\begin{aligned}
\mathscr{F}_{\text {loc }}(u, \Omega) & \leq \liminf _{k \rightarrow \infty} \mathscr{F}_{\text {loc }}\left(v_{k}, \Omega\right) \\
& \leq C\left(\mathscr{L}^{n}(\Omega)+\liminf _{k \rightarrow \infty}\left|D v_{k}\right|(\Omega)\right) \\
& =C\left(\mathscr{L}^{n}(\Omega)+|D u|(\Omega)\right) .
\end{aligned}
$$

This completes the proof for $f$ linearly coercive. 
Suppose $f$ does not satisfy (3.11). In this case, simply let $g: \mathbb{R}^{N \times n} \rightarrow \mathbb{R}$ be defined as

$$
g(\xi):=f(\xi)+|\xi|
$$

so $g$ does satisfy (3.11). Let $\mathscr{G}(u, \cdot)$ be the Lebesgue-Serrin extension associated with $g$. By Proposition 3.2, there exists a sequence $\left(u_{j}\right) \subset W_{\mathrm{loc}}^{1, r}\left(\Omega ; \mathbb{R}^{N}\right)$ such that $u_{j} \stackrel{*}{\rightarrow} u$ in BV and

$$
\lim _{j \rightarrow \infty} \int_{\Omega} g\left(\nabla u_{j}\right) \mathrm{d} x=\lim _{j \rightarrow \infty} \int_{\Omega} f\left(\nabla u_{j}\right)+\left|\nabla u_{j}\right| \mathrm{d} x=\mathscr{G}(u, \Omega) .
$$

But, by what we have just proved,

$$
\mathscr{G}(u, \Omega) \leq C\left(\mathscr{L}^{n}(\Omega)+|D u|(\Omega)\right),
$$

Now conclude by simply noting that

$$
\mathscr{F}_{\text {loc }}(u, \Omega) \leq \liminf _{j \rightarrow \infty} \int_{\Omega} f\left(\nabla u_{j}\right) \mathrm{d} x \leq \liminf _{j \rightarrow \infty} \int_{\Omega} g\left(\nabla u_{j}\right) \mathrm{d} x=\mathscr{G}(u, \Omega) .
$$

Remark 3.6. Localising the proof of this result, we also obtain the upper bound

$$
\mathscr{F}_{\text {loc }}(u, U) \leq C\left(\mathscr{L}^{n}(U)+|D u|(U)\right)
$$

for any open subset $U \subset \Omega$. Related work concerning SBV and polyhedral approximation may be found in $[4,7,9,12]$.

\subsection{Remark on the quadratic growth case}

Let us briefly consider the quadratic growth case, i.e. $r=2$ in (1.2). Due to the covering argument in our proof of Lemma 3.3, this borderline case cannot be incorporated. However, if this were possible, it would be a very strong result. It would show in particular that no lower semicontinuity result such as the one in the next section can be obtained in the quadratic growth case. For instance, if we let $\Omega=B, n=N=2$ and $f(\xi)=|\operatorname{det} \xi|$, then $f$ is continuous, polyconvex, and has quadratic growth but is 0 on matrices of rank $\leq 1$. If we were to obtain the upper bound (3.10) in this case, then we could consider the map $u(x)=\xi x$ to see that

$$
\mathscr{F}_{\text {loc }}(u, B) \leq C(|\xi|+1) .
$$

This would mean that we cannot have a lower semicontinuity result in the sequential weak* topology of BV, even when the limit map is affine, as this would imply

$$
|\operatorname{det} \xi| \leq C \mathscr{F}_{\text {loc }}(u, B)
$$

and so the determinant would have linear growth on general matrices, which would be a contradiction.

\section{LOWER SEMICONTINUITY IN BV}

In this section we provide the proof of the final main result of this paper. Now, $\Omega$ is a bounded, open subset of $\mathbb{R}^{n}, n \geq 2$ (not necessarily an extension domain), and we are considering the variational integral $F$ as defined in (1.1) and the Lebesgue-Serrin Extension $\mathscr{F}_{\text {loc }}$ as defined in (1.4); here the integrand $f$ satisfies (1.2) for $r \in\left[1, \frac{n}{n-1}\right)$ and is also assumed to be quasiconvex. Recall that the recession function $f^{\infty}$ of $f$ is defined as

$$
f^{\infty}(\xi):=\limsup _{t \rightarrow \infty} \frac{f(t \xi)}{t}
$$


We shall obtain a lower bound for $\mathscr{F}_{\text {loc }}$, provided we assume additionally that $f^{\infty}$ is finite in certain rank-one directions. That is, for a given $u \in \operatorname{BV}\left(\Omega ; \mathbb{R}^{N}\right)$,

$$
f^{\infty}(u(y) \otimes \nu)<\infty \quad \text { for } \mathscr{L}^{n} \text {-a.a. } y \in \Omega \text { and all } \nu \in \mathbb{R}^{n} .
$$

This is a natural assumption, since otherwise $f^{\infty}\left(D^{s} u /\left|D^{s} u\right|\right)$ may just be infinity for general BV functions. Henceforth, taking a suitable precise representative if necessary, we shall assume without loss of generality that (4.2) holds for all $y \in \Omega$. Note that since $f$ is quasiconvex, $f^{\infty}$ is rank-one convex (see, for example, [40]), meaning that it is finite also on rank-one matrices of the form $\xi=\eta \otimes \nu$ whenever $\nu \in \mathbb{R}^{n}$ and $\eta \in \operatorname{span}\{u(y)$ : $y \in \Omega\}$. Observe that the definition of the recession function immediately implies that $f$ has linear growth in any direction where $f^{\infty}$ is finite. This will allow us to apply the results of the previous section. Moreover, since $f^{\infty}(0)=0$, we have the linear growth condition

$$
f^{\infty}(\xi) \leq C|\xi|
$$

for a fixed finite $C>0$, for all $\xi \in \mathbb{R}^{N \times n}$ such that $\xi=\eta \otimes \nu, \eta \in \operatorname{span}\{u(y): y \in \Omega\}, \nu \in \mathbb{R}^{n}$.

It is also important to note that we are most interested in the case where

$$
\operatorname{span}\{u(y): y \in \Omega\} \neq \mathbb{R}^{N} .
$$

This is because, as we shall show in Section 4.3, there can be no non-negative quasiconvex function of genuinely $r$ growth for $1<r<\frac{n}{n-1}$ for which $f^{\infty}$ is finite in all rank-one directions: that is, if $f$ is quasiconvex and satisfies (1.2) for $1 \leq r<\frac{n}{n-1}$ but has linear growth for all matrices $\xi$ where $\operatorname{rank}(\xi) \leq 1$, then $f$ must in fact have linear growth in all directions. The proof of this is a straightforward application of Theorem 3.5 and a result of Kristensen [30].

The statement of the main theorem is as follows.

Theorem 4.1. Let $\Omega$ be a bounded, open set in $\mathbb{R}^{n}$ and $u \in B V\left(\Omega ; \mathbb{R}^{N}\right)$. Let $f: \mathbb{R}^{N \times n} \rightarrow \mathbb{R}$ be a quasiconvex function satisfying the growth condition (1.2) for $r \in\left[1, \frac{n}{n-1}\right)$. Let the recession function $f^{\infty}$ be as defined in (4.1), and suppose it is finite on rank-one matrices of the form $u(y) \otimes \nu, y \in \Omega, \nu \in \mathbb{R}^{n}$.

Suppose $\left(u_{j}\right)$ is a sequence in $W_{\text {loc }}^{1, r}\left(\Omega ; \mathbb{R}^{N}\right)$ such that

$$
u_{j} \stackrel{*}{\rightarrow} u \text { in } B V\left(\Omega ; \mathbb{R}^{N}\right) .
$$

Then

$$
\liminf _{j \rightarrow \infty} F\left(u_{j}, \Omega\right) \geq \int_{\Omega} f(\nabla u(x)) \mathrm{d} x+\int_{\Omega} f^{\infty}\left(\frac{D^{s} u}{\left|D^{s} u\right|}\right)\left|D^{s} u\right|,
$$

and hence

$$
\mathscr{F}_{\text {loc }}(u, \Omega) \geq \int_{\Omega} f(\nabla u(x)) \mathrm{d} x+\int_{\Omega} f^{\infty}\left(\frac{D^{s} u}{\left|D^{s} u\right|}\right)\left|D^{s} u\right| .
$$

A key property of $\mathscr{F}_{\text {loc }}$ that will be of use to us is that if it is finite, and $\Omega$ is an extension domain, then there exists a non-negative, finite Radon measure $\lambda$ on $\Omega$ such that

$$
\mathscr{F}_{\text {loc }}(u, U)=\lambda(U)
$$

for all open sets $U \subset \Omega$. That is, $\lambda$ (strongly) represents $\mathscr{F}_{\text {loc }}(u, \cdot)$ on $\Omega$. That is, we have the following theorem.

Theorem 4.2. Suppose $\Omega$ is an open, bounded extension domain in $\mathbb{R}^{n}, n \geq 2$. Let $f: \mathbb{R}^{N \times n} \rightarrow \mathbb{R}$ be a continuous function satisfying the growth condition (1.2) for some exponent $1 \leq r<\frac{n}{n-1}$. Let $u \in B V\left(\Omega ; \mathbb{R}^{N}\right)$ and $\mathscr{F}_{\mathrm{loc}}$ be as defined in (1.4). Then if $\mathscr{F}_{\mathrm{loc}}(u, \Omega)<\infty$, then there exists a non-negative, finite Radon measure $\lambda$ on $\Omega$ which represents $\mathscr{F}_{\text {loc }}$. 
This may be proved by straight-forwardly adapting a well-known result of Fonseca and Malý [24] to a borderline case. For completion and the reader's convenience, we include a proof in the appendix (Thm. A.5).

The structure of the rest of this section is as follows. First we show that the proof of this result involves establishing two inequalities: one on the absolutely continuous part of the measure $D u$, and one on the singlular part. The first inequality is essentially a direct application of a result by Kristensen in [30]. We prove the inequality on the singular part of $D u$ by obtaining a lower bound on $\mathscr{F}_{\text {loc }}$ via a technique of Ambrosio and Dal Maso [8], and combining this with a non-standard blow-up technique. Throughout the latter half of this section, we shall regularly make use of Theorem 4.2 and the upper bound in Theorem 3.5.

\subsection{Preliminaries}

Let $f$ be as stated in the assumptions of Theorem 4.1, and likewise let $\left(u_{j}\right)$ be a sequence in $W_{\text {loc }}^{1, r}\left(\Omega ; \mathbb{R}^{N}\right)$, $u \in \operatorname{BV}\left(\Omega ; \mathbb{R}^{N}\right)$, and $u_{j} \stackrel{*}{\rightarrow} u$ in $\operatorname{BV}\left(\Omega ; \mathbb{R}^{N}\right)$. We may assume that

$$
\liminf _{j \rightarrow \infty} F\left(u_{j}, \Omega\right)
$$

is finite, as otherwise there is nothing to prove. Moreover, by taking a subsequence (for convenience not relabelled), we can also assume

$$
\lim _{j \rightarrow \infty} F\left(u_{j}, \Omega\right)=\liminf _{j \rightarrow \infty} F\left(u_{j}, \Omega\right) .
$$

Thus the sequence $f\left(\nabla u_{j}\right) \mathscr{L}^{n}$ is bounded in $\mathscr{M}(\bar{\Omega})$, so we that have for some further subsequence (again not relabelled) there exists a measure $\mu$ in $\bar{\Omega}$ such that

$$
f\left(\nabla u_{j}\right) \stackrel{*}{\rightarrow} \mu \text { in } \mathscr{M}(\bar{\Omega}) .
$$

Clearly, since $f$ is non-negative, $\mu$ must also be a non-negative measure on $\bar{\Omega}$. Now observe that by applying the Radon-Nikodým Theorem twice, first with $\mu$ and Lebesgue measure, and then again on the singular part of $\mu$ and $\left|D^{s} u\right|$, we may decompose $\mu$ as

$$
\mu=\frac{\mathrm{d} \mu}{\mathrm{d} \mathscr{L}^{n}} \mathscr{L}^{n}+\frac{\mathrm{d} \mu}{\mathrm{d}\left|D^{s} u\right|}\left|D^{s} u\right|+\mu^{*}
$$

where $\mu^{*}$ is non-negative and singular with respect to both Lebesgue measure and $\left|D^{s} u\right|$. Hence

$$
\liminf _{j \rightarrow \infty} \int_{\Omega} f\left(\nabla u_{j}\right) \mathrm{d} x \geq \mu(\Omega)=\int_{\Omega} \frac{\mathrm{d} \mu}{\mathrm{d} \mathscr{L}^{n}} \mathrm{~d} x+\int_{\Omega} \frac{\mathrm{d} \mu}{\mathrm{d}\left|D^{s} u\right|} \mathrm{d}\left|D^{s} u\right|+\mu^{*}(\Omega) .
$$

Therefore the required lower bound will follow if we can show that

$$
\frac{\mathrm{d} \mu}{\mathrm{d} \mathscr{L}^{n}}(x) \geq f(\nabla(x)) \text { for } \mathscr{L}^{n} \text {-a.a. } x \in \Omega,
$$

and

$$
\frac{\mathrm{d} \mu}{\mathrm{d}\left|D^{s} u\right|}(x) \geq f^{\infty}\left(\frac{\mathrm{d} D^{s} u}{\mathrm{~d}\left|D^{s} u\right|}(x)\right) \text { for }\left|D^{s} u\right| \text {-a.a. } x \in \Omega .
$$

These two inequalities are the subject of the main propositions of this section. First, however, we state a lemma, attributable to Kristensen [30], that is particularly important for establishing (4.7), which in turn plays a role in aspects of the proof of (4.8). In the statement of this lemma and subsequently we denote by $B_{\varrho}$ the open ball in $\mathbb{R}^{n}$ centred on the origin with radius $\varrho$, and $B=B_{1}$. 
Lemma 4.3 [30]. Let $f: \mathbb{R}^{N \times n} \rightarrow \mathbb{R}$ be a quasiconvex function satisfying the growth condition (1.2) for some exponent $r \in\left[1, \frac{n}{n-1}\right)$.

Let $\left(u_{j}\right)$ be a sequence in $W^{1, r}\left(B ; \mathbb{R}^{N}\right)$ and suppose

$$
u_{j} \rightarrow 0 \text { in } L^{1}\left(B ; \mathbb{R}^{N}\right)
$$

and

$$
\sup _{j} \int_{B}\left|\nabla u_{j}\right| \mathrm{d} x<+\infty
$$

Then we have the following inequality:

$$
\liminf _{j \rightarrow \infty} \int_{B} f\left(\nabla u_{j}\right) \mathrm{d} x \geq \mathscr{L}^{n}(B) \cdot f(0)
$$

The proof of Lemma 4.3 involves applying the following result, which gives us higher integrability for a tracepreserving extension operator.

Lemma 4.4 [30]. Let $1 \leq r<\frac{n}{n-1}$. Then there exists a linear extension operator

$$
\mathbf{E}:\left(W^{1,1}\right)\left(\partial B ; \mathbb{R}^{N}\right) \rightarrow W^{1, r}\left(B_{2} \backslash \bar{B} ; \mathbb{R}^{N}\right)
$$

with the following properties:

1. If $g \in C^{1}\left(\partial B ; \mathbb{R}^{N}\right)$ then $\mathbf{E}(g) \in C^{\infty}\left(B_{2} \backslash \bar{B}\right)$ with $\left.\mathbf{E}(g)\right|_{\partial B}=g$.

2. If $\left(z_{j}\right) \subset C^{\infty}\left(\partial B ; \mathbb{R}^{N}\right)$ and $\lim _{j \rightarrow \infty} \int_{\partial B} z_{j} \cdot \phi \mathrm{d} \mathscr{H}^{n-1}=0$ for all $\phi \in C^{\infty}\left(\partial B ; \mathbb{R}^{N}\right)$, then for any multi-index $\alpha, \partial^{\alpha}\left[\mathbf{E} z_{j}\right] \rightarrow 0$ locally uniformly in $B_{2} \backslash \bar{B}$.

3. There exist positive constants $c_{1}, c_{2}$, dependent on $n, N, r$, such that:

(a)

$$
\int_{B_{2} \backslash B}|\mathbf{E}(g)|^{r} \leq c_{1}\|g\|_{L^{1}(\partial B)}^{r}
$$

(b)

$$
\int_{B_{2} \backslash B}|\nabla[\mathbf{E} g]|^{r} \mathscr{L}^{n} \leq\left(c_{2} \int_{\partial B}|\nabla g| \mathrm{d} \mathscr{H}^{n-1}\right)^{r}
$$

for all $g \in C^{1}(\partial B)$.

Proof of Lemma 4.3. By approximation we may assume $\left(u_{j}\right) \subset C^{1}\left(\bar{B} ; \mathbb{R}^{N}\right)$. If the left hand side of $(4.11)$ is infinite then there is nothing to prove, so suppose it is finite. Moreover, by extracting a subsequence if necessary, we can assume

$$
l_{0}:=\liminf _{j \rightarrow \infty} \int_{B} f\left(\nabla u_{j}\right) \mathrm{d} x=\lim _{j \rightarrow \infty} \int_{B} f\left(\nabla u_{j}\right) \mathrm{d} x .
$$

From (4.9), by the Fubini-Tonelli theorem and the Rellich-Kondrachov compactness theorem we have

$$
\lim _{j \rightarrow \infty} \int_{0}^{1} \int_{\partial B_{\varrho}}\left|u_{j}\right| \mathrm{d} \mathscr{H}^{n-1} \mathrm{~d} \varrho=\lim _{j \rightarrow \infty} \int_{B}\left|u_{j}\right| \mathrm{d} x=0 .
$$

This implies there exists a subsequence $\left\{u_{j}\right\}_{j \in T}$ such that

$$
\lim _{j \rightarrow \infty, j \in T} \int_{\partial B_{\varrho}}\left|u_{j}\right| \mathrm{d} \mathscr{H}^{n-1}=0
$$


for almost all $\varrho \in(0,1)$. By Fatou's Lemma and (4.10) we have

$$
\int_{0}^{1} \liminf _{j \rightarrow \infty, j \in T} \int_{\partial B_{\varrho}}\left|\nabla u_{j}\right| \mathrm{d} \mathscr{H}^{n-1} \mathrm{~d} \varrho \leq \liminf _{j \rightarrow \infty} \int_{j \in T}\left|\nabla u_{j}\right| \mathrm{d} x<\infty .
$$

Thus, for almost all $\varrho \in(0,1)$

$$
\liminf _{j \rightarrow \infty, j \in T} \int_{\partial B_{\varrho}}\left|\nabla u_{j}\right| \mathrm{d} \mathscr{H}^{n-1}<\infty .
$$

Now fix $0<\delta<1$. By (4.12) and (4.13) we can choose $\varrho \in(\delta, 1)$ such that

$$
\lim _{j \rightarrow \infty, j \in T} \int_{\partial B_{\varrho}}\left|u_{j}\right| \mathrm{d} \mathscr{H}^{n-1}=0
$$

and

$$
\liminf _{j \rightarrow \infty} \int_{\partial B_{\varrho}}\left|\nabla u_{j}\right| \mathrm{d} \mathscr{H}^{n-1}<\infty .
$$

Now take a further subsequence $\left\{u_{j}\right\}_{j \in S}$, where $S \subseteq T$, so that

$$
\lim _{j \rightarrow \infty, j \in S} \int_{\partial B_{\varrho}}\left|\nabla u_{j}\right| \mathrm{d} \mathscr{H}^{n-1}=\liminf _{j \rightarrow \infty} \int_{\partial B_{\varrho}}\left|\nabla u_{j}\right| \mathrm{d} \mathscr{H}^{n-1} .
$$

Relabel the sequence $\left(u_{j}\right)$ so that $S=\mathbb{N}$. Now define the sequence $\left(g_{j}\right) \subset W^{1,1}\left(\partial B ; \mathbb{R}^{N}\right)$ as:

$$
g_{j}(x):=\left.u_{j}\right|_{\partial B_{\varrho}}(\varrho x) \text { for } x \in \partial B .
$$

Take a cut-off function $\eta \in C^{1}(B ; \mathbb{R})$ such that $\mathbf{1}_{B_{\varrho}} \leq \eta \leq \mathbf{1}_{B},|\nabla \eta| \leq \frac{2}{1-\varrho}$, and define $\left(v_{j}\right) \subset W_{0}^{1, r}\left(B ; \mathbb{R}^{N}\right)$ as:

$$
v_{j}(x):=\left\{\begin{array}{cc}
\eta(x) \cdot\left(\mathbf{E}\left(g_{j}\right)\right)\left(\frac{x}{\varrho}\right) & \text { if }|x| \geq \varrho \\
u_{j}(x) & \text { if }|x|<\varrho
\end{array}\right.
$$

where $\mathbf{E}$ is the extension operator from Lemma 4.4 .

Since the function $t \mapsto t^{r}$ is convex, $(s+t)^{r} \leq 2^{r-1}\left(s^{r}+t^{r}\right)$ for all $s, t \geq 0$. Hence from Lemma 4.4 we have

$$
\begin{aligned}
\int_{B \backslash B_{\varrho}}\left|\nabla v_{j}\right|^{r} & \leq \int_{B \backslash B_{\varrho}}\left(\left|\nabla \eta \cdot \mathbf{E} g_{j}(\cdot / \varrho)\right|+\left|\eta \cdot \nabla\left[\mathbf{E} g_{j}(\cdot / \varrho)\right]\right|\right)^{r} \\
& \leq 2^{r-1} \int_{B \backslash B_{\varrho}}|\nabla \eta|^{r} \cdot\left|\mathbf{E} g_{j}(\cdot / \varrho)\right|^{r}+2^{r-1} \int_{B \backslash B_{\varrho}}|\eta|^{r} \cdot\left|\nabla\left[\mathbf{E} g_{j}(\cdot / \varrho)\right]\right|^{r} \\
& \leq C \int_{B \backslash B_{\varrho}}\left|\mathbf{E} g_{j}(\cdot / \varrho)\right|^{r}+C \int_{B \backslash B_{\varrho}}\left|\nabla\left[\mathbf{E} g_{j}(\cdot / \varrho)\right]\right|^{r}
\end{aligned}
$$

for some constant C. We estimate the two terms in (4.14) using Lemma 4.4 (3) as follows. Firstly, note that we have

$$
\begin{aligned}
\int_{B \backslash B_{\varrho}}\left|\left[\mathbf{E} g_{j}(\cdot / \varrho)\right]\right|^{r} & \leq c_{1}\left\|g_{j}\right\|_{L^{1}(\partial B)}^{r} \\
& =c_{1}\left\|u_{j}\right\|_{L^{1}\left(\partial B_{\varrho}\right)}^{r} \\
& \rightarrow 0 \text { as } j \rightarrow \infty .
\end{aligned}
$$


Now we use (3)(b) to estimate the remaining term:

$$
\begin{aligned}
\int_{B \backslash B_{\varrho}}\left|\nabla\left[\mathbf{E} g_{j}(\cdot / \varrho)\right]\right|^{r} & \leq\left(c_{2} \int_{\partial B}\left|\nabla g_{j}\right| \mathrm{d} \mathscr{H}^{n-1}\right)^{r} \\
& =\left(c_{2} \int_{\partial B_{\varrho}}\left|\nabla u_{j}\right| \mathrm{d} \mathscr{H}^{n-1}\right)^{r} .
\end{aligned}
$$

Now note that we may obtain the same inequality (albeit for a different constant) using Lemma 4.4 for any other $r^{\prime}$ such that $r<r^{\prime}<\frac{n}{n-1}$. Hence by (4.15) and Lemma 4.4, since

$$
\sup _{j} \int_{\partial B_{\varrho}}\left|\nabla u_{j}\right| \mathrm{d} \mathscr{H}^{n-1}<\infty
$$

we can use the De la Vallée Poussin criterion to deduce that the sequence $\left|\nabla\left[\mathbf{E} g_{j}\right]\right|^{r}$ is equi-integrable on $B \backslash B_{\varrho}$. By Lemma 4.4, since

$$
\sup _{j} \int_{\partial B_{\varrho}}\left|u_{j}\right| \mathrm{d} \mathscr{H}^{n-1} \rightarrow 0 \text { as } j \rightarrow \infty
$$

$\nabla\left[\mathbf{E} g_{j}\right] \rightarrow 0$ locally uniformly on $B \backslash B_{\varrho}$, and hence so does $\left|\nabla\left[\mathbf{E} g_{j}\right]\right|^{r}$. Thus, by Vitali's Convergence Theorem,

$$
\int_{B \backslash B_{\varrho}}\left|\nabla\left[\mathbf{E} g_{j}(\cdot / \varrho)\right]\right|^{r} \rightarrow 0 \quad \text { as } j \rightarrow \infty .
$$

Combining these estimates in (4.14), we have

$$
\limsup _{j \rightarrow \infty} \int_{B \backslash B_{\varrho}}\left|\nabla v_{j}\right|^{r} \mathrm{~d} x=0 .
$$

Now we use the quasiconvexity and non-negativity of $f$ to obtain

$$
\begin{aligned}
\int_{B} f\left(\nabla u_{j}\right) \geq \int_{B_{\varrho}} f\left(\nabla u_{j}\right) & =\int_{B} f\left(\nabla v_{j}\right)-\int_{B \backslash B_{\varrho}} f\left(\nabla v_{j}\right) \\
& \geq \mathscr{L}^{n}(B) f(0)-\int_{B \backslash B_{\varrho}} f\left(\nabla v_{j}\right) \\
& \geq \mathscr{L}^{n}(B) f(0)-L \int_{B \backslash B_{\varrho}}\left(1+\left|\nabla v_{j}\right|^{r}\right) .
\end{aligned}
$$

Let $j \rightarrow \infty$ to get, using (4.16),

$$
l_{0} \geq \mathscr{L}^{n}(B) f(0)-L \mathscr{L}^{n}\left(B \backslash B_{\varrho}\right) .
$$

Recall $\varrho \in(\delta, 1)$ for fixed $0<\delta<1$. Hence we conclude by taking $\delta$ arbitrarily close to 1 , which completes the proof of the Lemma.

\subsection{Lower bound on the absolutely continuous part}

We now state and prove (4.7), which is essentially just the lower semicontinuity result proved by Kristensen in [30]. Note that it does not require any finiteness properties of $f^{\infty}-$ in fact $f^{\infty}$ does not feature at all in this context.

Proposition 4.5. Let $f: \mathbb{R}^{N \times n} \rightarrow \mathbb{R}$ be a quasiconvex function satisfying the growth condition (1.2) for some exponent $1 \leq r<\frac{n}{n-1}$. Let $\Omega$ be a bounded, open subset of $\mathbb{R}^{n}$. 
Let $\left(u_{j}\right)$ be a sequence in $W_{\mathrm{loc}}^{1, r}\left(\Omega ; \mathbb{R}^{N}\right)$ and $u \in B V\left(\Omega ; \mathbb{R}^{N}\right)$. Suppose

$$
u_{j} \stackrel{*}{\rightarrow} u \text { in } B V\left(\Omega ; \mathbb{R}^{N}\right) .
$$

Let $\mu$ be a measure in $\bar{\Omega}$ and suppose

$$
f\left(\nabla u_{j}\right) \stackrel{*}{\rightarrow} \mu \text { in } \mathscr{M}(\bar{\Omega})
$$

Then for $\mathscr{L}^{n}$-almost all $x \in \Omega$, we have

$$
\frac{\mathrm{d} \mu}{\mathrm{d} \mathscr{L}^{n}}(x) \geq f(\nabla(x)) .
$$

The proof of Proposition 4.5 is just a straightforward blow-up argument using Lemma 4.3.

Proof of Proposition 4.5. Since, by (4.17) and the Uniform Boundedness Principle, $\left|\nabla u_{j}\right| \mathscr{L}^{n}$ is bounded in $\mathscr{M}(\bar{\Omega})$, we have for some subsequence (for convenience not relabelled) that there exists a measure $\nu$ in $\bar{\Omega}$ such that

$$
\left|\nabla u_{j}\right| \stackrel{*}{\rightarrow} \nu \quad \text { in } \mathscr{M}(\bar{\Omega}) .
$$

Let $\Omega_{0}$ denote the set of points $x \in \Omega$ such that

1.

$$
\frac{\mathrm{d} \mu}{\mathrm{d} \mathscr{L}^{n}}(x)=\lim _{\varrho \rightarrow 0^{+}} \frac{\mu(\overline{B(x, \varrho)})}{\mathscr{L}^{n}(B(x, \varrho))} \quad \text { exists and is finite }
$$

2.

$$
\frac{\mathrm{d} \nu}{\mathrm{d} \mathscr{L}^{n}}(x)=\lim _{\varrho \rightarrow 0^{+}} \frac{\nu(\overline{B(x, \varrho)})}{\mathscr{L}^{n}(B(x, \varrho))} \quad \text { exists and is finite }
$$

3.

$$
\lim _{\varrho \rightarrow 0^{+}} \frac{1}{\varrho} f_{B(x, \varrho)}|u(y)-u(x)-[\nabla u(x)](x-y)| \mathrm{d} y=0
$$

where $\nabla u$ is the Radon-Nikodým derivative of $D u$ with respect to Lebesgue measure. By standard results (see for example $[43,49]), \mathscr{L}^{n}\left(\Omega \backslash \Omega_{0}\right)=0$. Fix $x_{0} \in \Omega_{0}$, and note that since the set

$$
\{\varrho \in(0, \operatorname{dist}(x, \partial \Omega)):(\mu+\nu)(\partial B(x, \varrho))>0\}
$$

is at most countable we may find a sequence $r_{k} \searrow 0$ such that $(\mu+\nu)\left(\partial B\left(x, r_{k}\right)\right)=0$ for all $k$. Now define

$$
v_{j, k}(y):=\frac{u_{j}\left(x_{0}+r_{k} y\right)-u\left(x_{0}\right)-\left[\nabla u\left(x_{0}\right)\right]\left(r_{k} y\right)}{r_{k}}, \quad y \in B .
$$

Then by the above assumptions we have

$$
\begin{aligned}
\lim _{k \rightarrow \infty} \lim _{j \rightarrow \infty} \int_{B} \mid & v_{j, k}(y) \mid \mathrm{d} y=0 \\
\lim _{k \rightarrow \infty} \lim _{j \rightarrow \infty} f_{B}\left|\nabla v_{j, k}(y)+\nabla u\left(x_{0}\right)\right| \mathrm{d} y & =\lim _{k \rightarrow \infty} \lim _{j \rightarrow \infty} f_{B}\left|\nabla u_{j}\left(x_{0}+r_{k} y\right)\right| \mathrm{d} y \\
& =\lim _{k \rightarrow \infty} \frac{1}{\left|B\left(x_{0}, r_{k}\right)\right|} \int_{B\left(x_{0}, r_{k}\right)}|\nabla u(y)| \mathrm{d} y \\
& =\frac{\mathrm{d} \nu}{\mathrm{d} \mathscr{L}^{n}}\left(x_{0}\right),
\end{aligned}
$$


and similarly

$$
\lim _{k \rightarrow \infty} \lim _{j \rightarrow \infty} f_{B} f\left(\nabla v_{j, k}(y)+\nabla u\left(x_{0}\right)\right) \mathrm{d} y=\frac{\mathrm{d} \mu}{\mathrm{d} \mathscr{L}^{n}}\left(x_{0}\right) .
$$

Hence for each $k$ we can find $j_{k} \in \mathbb{N}$ such that the all the convergence above occurs for $v_{j_{k}, k}$ as $k$ tends to infinity. Thus, if we define $z_{k}:=v_{j_{k}, k}$, then $\left(z_{k}\right) \subset W^{1, r}\left(B ; \mathbb{R}^{N}\right)$ satisfies conditions (4.9) and (4.10) of Lemma 4.3. Applying this lemma (to the function $\bar{f}(\xi)=f\left(\nabla u\left(x_{0}\right)+\xi\right)$, say), we obtain

$$
\liminf _{k \rightarrow \infty} f_{B} f\left(\nabla z_{k}+\nabla u\left(x_{0}\right)\right) \mathrm{d} y \geq f\left(\nabla u\left(x_{0}\right)\right),
$$

i.e.

$$
\frac{\mathrm{d} \mu}{\mathrm{d} \mathscr{L}^{n}}\left(x_{0}\right) \geq f\left(\nabla\left(x_{0}\right)\right)
$$

as required.

Now we remark that the following result follows immediately from this proposition by integrating $\frac{\mathrm{d} \mu}{\mathrm{d} x}$ with respect to Lebesgue measure over $\Omega$. It gives a first lower bound for the Lebesgue-Serrin extension, which Theorem 4.1 improves upon, provided additional assumptions on $f$ are satisfied.

Corollary 4.6. Let $f: \mathbb{R}^{N \times n} \rightarrow \mathbb{R}$ be a quasiconvex function satisfying the growth condition (1.2) for some exponent $1 \leq r<\frac{n}{n-1}$. Let $\Omega$ be a bounded, open subset of $\mathbb{R}^{n}$.

Let $\left(u_{j}\right)$ be a sequence in $W_{\text {loc }}^{1, r}\left(\Omega ; \mathbb{R}^{N}\right)$ and $u \in B V\left(\Omega ; \mathbb{R}^{N}\right)$. Suppose

$$
u_{j} \stackrel{*}{\rightarrow} u \text { in } B V\left(\Omega ; \mathbb{R}^{N}\right) .
$$

Then

$$
\liminf _{j \rightarrow \infty} \int_{\Omega} f\left(\nabla u_{j}\right) \mathrm{d} x \geq \int_{\Omega} f(\nabla u) \mathrm{d} x
$$

and hence

$$
\mathscr{F}_{\text {loc }}(u, \Omega) \geq \int_{\Omega} f(\nabla u) \mathrm{d} x
$$

\subsection{Remarks on linear growth on full rank-one cone}

We now observe that upper and lower bounds in Theorem 3.5 and Corollary 4.6 respectively enable us to show that whenever $f$ is quasiconvex and satisfies (1.2) for $1 \leq r<\frac{n}{n-1}$, then finiteness of $f^{\infty}$ on the full rank-one cone in fact implies $f$ has at most linear growth in all directions. In other words, there can be no non-negative quasiconvex function which has genuinely superlinear growth $1<r<\frac{n}{n-1}$ but only linear growth in rank-one directions.

Corollary 4.7. Let $f: \mathbb{R}^{N \times n} \rightarrow \mathbb{R}$ be a quasiconvex function satisfying the growth condition (1.2) for some exponent $1 \leq r<\frac{n}{n-1}$. Suppose $f$ has linear growth on matrices of rank at most one, i.e.

$$
0 \leq f(\xi) \leq M(|\xi|+1)
$$

for a fixed finite $M>0$ and all $\xi \in \mathbb{R}^{N \times n}$ satisfying $\operatorname{rank}(\xi) \leq 1$. Then $f$ has linear growth in all directions, i.e. (4.19) holds for all $\xi \in \mathbb{R}^{N \times n}$ (for perhaps a larger constant).

Proof of Corollary 4.7. Let $B$ denote the open unit ball in $\mathbb{R}^{n}$. Let $\xi$ be a general matrix in $\mathbb{R}^{N \times n}$, and consider the map $u(x)=\xi x$. Then, since $f$ certainly satisfies the conditions of Theorem 3.5 , and $D u=\nabla u=\xi \mathscr{L}^{n}$, we have

$$
\mathscr{F}_{\text {loc }}(u, B) \leq C(|\xi|+1)
$$


for some constant $C$ independent of $\xi$. However, by Corollary 4.6 we also have

$$
\mathscr{F}_{\text {loc }}(u, B) \geq \int_{B} f(\nabla u) \mathrm{d} x=\mathscr{L}^{n}(B) f(\xi) .
$$

Combining these two bounds, we get

$$
f(\xi) \leq C(|\xi|+1)
$$

from where the result follows, since $C$ is independent of $\xi$, which is arbitrary.

Moreover, as the following proposition indicates, we believe that this result can be generalised to the case where $f$ is $W^{1, r}$-quasiconvex for $1 \leq r<2$, has linear growth on the rank-one cone, but otherwise need satisfy no other growth conditions on general matrices. Although our result is limited to the case $n=N=2$, we believe that it ought to be possible to generalise this result for higher dimensions, and are currently working on this.

Recall that $f: \mathbb{R}^{N \times n} \rightarrow \mathbb{R}$ is said to be $W^{1, r}$-quasiconvex for some exponent $1 \leq r \leq \infty$ if it satisfies the inequality

$$
\int_{B} f(\xi+\nabla \phi(x)) \mathrm{d} x \geq \mathscr{L}^{n}(B) f(\xi)
$$

for all $\xi \in \mathbb{R}^{N \times n}$ and all $\phi \in W_{0}^{1, r}\left(B ; \mathbb{R}^{N}\right)$. This definition was introduced and first studied by Ball and Murat in [14], and is generalisation of Morrey's classical notion of quasiconvexity. We remark that if $f$ is quasiconvex and satisfies the growth condition (1.2) for some exponent $r$, then it is $W^{1, r}$-quasiconvex.

Proposition 4.8. Let $n=N=2$, and $B$ denote the open unit ball in $\mathbb{R}^{2}$. Let $1<r<2$ and $f: \mathbb{R}^{2 \times 2} \rightarrow \mathbb{R}$ be a non-negative $W^{1, r}$-quasiconvex function.

Suppose $f$ has linear growth on matrices of rank at most one, i.e. it satisfies (4.19) for a fixed finite $M>0$ and all $\xi \in \mathbb{R}^{2 \times 2}$ satisfying $\operatorname{rank}(\xi) \leq 1$. Then $f$ has linear growth from above in all directions, i.e. (4.19) holds for all $\xi \in \mathbb{R}^{2 \times 2}$ (for perhaps a larger constant).

Proof of Proposition 4.8. Let $\xi \in \mathbb{R}^{2 \times 2}$. Now define the map $u_{\xi}: B \rightarrow \mathbb{R}^{2}$ as

$$
u_{\xi}(x)=\frac{\xi x}{|x|}
$$

Note that $u_{\xi}$ maps $B \backslash\{0\}$ to the surface $\xi(\partial B)$, so $\operatorname{det}\left(\nabla u_{\xi}(x)\right)=0$ for all $x \in B \backslash\{0\}$. Hence $\operatorname{rank}\left(\operatorname{det}\left(\nabla u_{\xi}\right)\right) \leq 1$ on $B \backslash\{0\}$. Indeed,

$$
\begin{aligned}
\nabla u_{\xi}\left(x_{1}, x_{2}\right) & =\frac{\xi}{\left(x_{1}^{2}+x_{2}^{2}\right)^{\frac{3}{2}}} \cdot\left(\begin{array}{cc}
x_{2}^{2} & -x_{1} x_{2} \\
-x_{1} x_{2} & x_{1}^{2}
\end{array}\right) \\
& =\frac{\xi}{\left(x_{1}^{2}+x_{2}^{2}\right)^{\frac{3}{2}}} \cdot\left(\left(x_{2},-x_{1}\right)^{t} \otimes\left(x_{2},-x_{1}\right)^{t}\right) .
\end{aligned}
$$

Hence, by assumption,

$$
f\left(\nabla u_{\xi}(x)\right) \leq M\left(\left|\nabla u_{\xi}(x)\right|+1\right)
$$

for all $x \in B \backslash\{0\}$. It is well-known that $u_{\xi} \in W^{1, q}\left(B ; \mathbb{R}^{2}\right)$ for all $1 \leq q<n=2$ when $\xi$ is the identity (see for example [14]), and consequently clearly also for any other $\xi \in \mathbb{R}^{2 \times 2}$. Moreover, $u_{\xi}(x)=\xi x$ on $\partial B$. Therefore, since $f$ is $W^{1, r}$-quasiconvex and $1<r<2$, we have

$$
\int_{B} f\left(\nabla u_{\xi}\right) \mathrm{d} x \geq \mathscr{L}^{2}(B) f(\xi)
$$


Thus, using (4.20) and (4.21), we get

$$
\begin{aligned}
f(\xi) & \leq(M /|B|) \int_{B} 1+\left|\nabla u_{\xi}\right| \mathrm{d} x \\
& \leq(M /|B|)\left(1+\int_{B}|\xi(\nabla(x /|x|))| \mathrm{d} x\right) \\
& \leq(M /|B|)\left(1+|\xi| \int_{B}|\nabla(x /|x|)| \mathrm{d} x\right) .
\end{aligned}
$$

Since $x \mapsto x /|x|$ is in $W^{1,1}\left(B ; \mathbb{R}^{2}\right)$, the required result follows with $M$ replaced by $(M /|B|) \int_{B}|\nabla(x /|x|)| \mathrm{d} x=$ $2 M$.

\subsection{Lower bound on the singular part}

Theorem 2.4 plays a key part in our proof of the inequality (4.8). This is because it shows us that the blow-up of a BV function on the singular part of the derivative is essentially a function of one variable; this allows us to apply the following lemma, similar to one of Ambrosio and Dal Maso [8], which gives us a useful lower bound for the Lebesgue-Serrin extension of such functions.

Lemma 4.9. Let $Q \subset \mathbb{R}^{n}$ be a unit $n$-cube whose sides are either orthogonal or parallel to a unit vector $\nu \in \mathbb{R}^{n}$, let $\eta$ be a unit vector in $\mathbb{R}^{N}$, and let $v \in B V\left(Q ; \mathbb{R}^{N}\right)$ be a function representable as

$$
v(y)=\psi(\langle y, \nu\rangle) \eta
$$

for a some non-decreasing function $\psi:(a, b) \rightarrow \mathbb{R}$. Suppose $u \in B V\left(Q ; \mathbb{R}^{N}\right)$ satisfies $\operatorname{supp}(v-u) \subset \subset Q$.

Let $f: \mathbb{R}^{N \times n} \rightarrow \mathbb{R}$ be a quasiconvex function satisfying the growth condition (1.2) for some exponent $1 \leq r<$ $\frac{n}{n-1}$, and also the coercivity condition

$$
f(\xi) \geq c_{0}|\xi|
$$

for some constant $c_{0}>0$, for all $\xi \in \mathbb{R}^{N \times n}$. Let the recession function $f^{\infty}$ be as defined in (4.1), and suppose it is finite on rank-one matrices of the form $u(y) \otimes \nu, y \in \Omega, \nu \in \mathbb{R}^{n}$. Let $\mathscr{F}_{\text {loc }}$ be as defined in (1.4). Then

$$
\mathscr{F}_{\text {loc }}(u, Q) \geq f(D u(Q)) \text {. }
$$

Proof of Lemma 4.9. We may assume without loss of generality that $\nu=e_{1}$ and $Q=(0,1)^{n}$. Let $\psi:(0,1) \rightarrow \mathbb{R}$ be a non-decreasing function such that $v(y)=\psi\left(y_{1}\right) \eta$, and let $\alpha$ denote the increment of $\psi$ in $(0,1)$, i.e.

$$
\alpha=\lim _{t \rightarrow 1^{-}} \psi(t)-\lim _{t \rightarrow 0^{+}} \psi(t)=|D \psi|(0,1)=|D v|(Q)<+\infty .
$$

Now define $w \in \mathrm{BV}_{\text {loc }}\left((0,+\infty)^{n} ; \mathbb{R}^{N}\right)$ by

$$
w(y):=u(y-[y])+\alpha\left[y_{1}\right] \eta,
$$

where, for every $t \in \mathbb{R},[t]$ denotes the integer part of $t$, and for $y=\left(y_{1}, \ldots, y_{n}\right) \in \mathbb{R}^{n},[y]$ is defined to be $\left(\left[y_{1}\right], \ldots,\left[y_{n}\right]\right)$. Now define, for $y \in Q$,

$$
u_{k}(y):=\frac{w(k y)}{k} \in \operatorname{BV}\left(Q ; \mathbb{R}^{N}\right) .
$$

Note that

$$
u_{k}(y)=\frac{u(k y-[k y])}{k}+\alpha \frac{\left[k y_{1}\right]}{k} \eta
$$


$\left[k y_{1}\right] / k$ converges to $y_{1}$ as $k \rightarrow \infty$, and

$$
\int_{Q}\left|\frac{u(k y-[k y])}{k}\right| \mathrm{d} y=\frac{1}{k^{n+1}} \int_{(0, k)^{n}}|u(y-[y])| \mathrm{d} y=\frac{1}{k} \int_{Q}|u(y)| \mathrm{d} y \rightarrow 0 .
$$

Therefore $u_{k}$ converges to the affine function $u_{0}(y)=\alpha y_{1} \eta$ in $L^{1}\left(Q ; \mathbb{R}^{N}\right)$. Now let $Q_{1} \ldots Q_{k^{n}}$ be the standard decomposition of $Q$ into $k^{n}$ congruent cubes of side length $1 / k$. Since, by construction, $D w$ does not have any jumps on any hyperplane of the form $y_{j}=h$ where $h$ is an integer and $1 \leq j \leq n$, it follows that

$$
\left|D u_{k}\right|\left(Q \cap \partial\left(Q_{i}\right)\right)=0 \quad \text { for all } 1 \leq i \leq k^{n} .
$$

This implies

$$
D u_{k}(Q)=D w(Q)=D u(Q),
$$

so $\left(u_{k}\right)$ is bounded in $\mathrm{BV}\left(Q ; \mathbb{R}^{N}\right)$. Hence in fact the sequence converges weakly* in BV to $u_{0}$. By Proposition 3.1 , we get

$$
\mathscr{F}_{\text {loc }}\left(u_{k},(0,1 / k)^{n}\right)=(1 / k)^{n} \mathscr{F}_{\text {loc }}(u, Q), \quad \mathscr{F}_{\text {loc }}\left(u_{k},(0,1 / k)^{n}\right)=\mathscr{F}_{\text {loc }}\left(u_{k}, Q_{i}\right)
$$

for all $1 \leq i \leq k^{n}$. Now, using the fact that $\mathscr{F}_{\text {loc }}$ has a measure representation, the upper bound (3.12) from Theorem 3.5, and (4.22), it follows that

$$
\mathscr{F}_{\text {loc }}\left(u_{k}, Q\right)=\sum_{i=1}^{k^{n}} \mathscr{F}_{\text {loc }}\left(u_{k}, Q_{i}\right) .
$$

This implies, together with (4.23), that $\mathscr{F}_{\mathrm{loc}}\left(u_{k}, Q\right)=\mathscr{F}_{\mathrm{loc}}(u, Q)$. By Corollary 4.6 and the lower semicontinuity of $\mathscr{F}_{\text {loc }}$, we get

$$
\mathscr{F}_{\mathrm{loc}}(u, Q)=\lim _{k \rightarrow \infty} \mathscr{F}_{\mathrm{loc}}\left(u_{k}, Q\right) \geq \mathscr{F}_{\mathrm{loc}}\left(u_{0}, Q\right) \geq f\left(\alpha \eta \otimes e_{1}\right) .
$$

Noting that $D u(Q)=D v(Q)=\alpha \eta \otimes e_{1}$, the proof is complete.

We are now in a position to be able to prove the inequality (4.8) which, combined with the proof of (4.7) established in Proposition 4.5, allows us to conclude our proof of Theorem 4.1. In order to use the results of the previous section, we first need to assume that the integrand $f$ is coercive, before then showing how this assumption can be removed.

Proposition 4.10. Let $\Omega$ be a bounded, open subset of $\mathbb{R}^{n}$, and let $\left(u_{j}\right)$ be a sequence in $W_{\text {loc }}^{1, r}\left(\Omega ; \mathbb{R}^{N}\right)$ and $u \in B V\left(\Omega ; \mathbb{R}^{N}\right)$. Let $f: \mathbb{R}^{N \times n} \rightarrow \mathbb{R}$ be a quasiconvex function satisfying the growth condition (1.2) for some exponent $1 \leq r<\frac{n}{n-1}$, that also satisfies the coercivity condition

$$
f(\xi) \geq c_{0}|\xi|
$$

for some constant $c_{0}>0$, for all $\xi \in \mathbb{R}^{N \times n}$. Let the recession function $f^{\infty}$ be as defined in (4.1), and suppose it is finite on rank-one matrices of the form $u(y) \otimes \nu, y \in \Omega, \nu \in \mathbb{R}^{n}$.

Suppose

$$
u_{j} \stackrel{*}{\rightarrow} u \text { in } B V\left(\Omega ; \mathbb{R}^{N}\right) \text {. }
$$

Let $\mu$ be a measure in $\bar{\Omega}$ and suppose

$$
f\left(\nabla u_{j}\right) \stackrel{*}{\rightarrow} \mu \text { in } \mathscr{M}(\bar{\Omega}) .
$$

Then for $\left|D^{s} u\right|$-almost all $x \in \Omega$, we have

$$
\frac{\mathrm{d} \mu}{\mathrm{d}\left|D^{s} u\right|}(x) \geq f^{\infty}\left(\frac{D^{s} u}{\left|D^{s} u\right|}(x)\right) .
$$


Proof of Proposition 4.10. By Theorem 2.4, letting $\xi: \Omega \rightarrow \mathbb{R}^{N \times n}$ denote the density of $D u$ with respect to $|D u|$, we have, for $\left|D^{s} u\right|$-almost all $x_{0} \in \Omega,\left|\xi\left(x_{0}\right)\right|=1, \operatorname{rank}\left(\xi\left(x_{0}\right)\right)=1$, and

$$
\lim _{\varrho \rightarrow 0^{+}} \frac{D u\left(Q\left(x_{0}, \varrho\right)\right)}{|D u|\left(Q\left(x_{0}, \varrho\right)\right)}=\xi\left(x_{0}\right), \quad \lim _{\varrho \rightarrow 0^{+}} \frac{D u\left(Q\left(x_{0}, \varrho\right)\right)}{\varrho^{n}}=+\infty,
$$

where $Q\left(x_{0}, \varrho\right)$ is any cube centred at $x_{0}$ with side-length $\varrho$. Fix $x_{0}$ with these properties, and write $\xi\left(x_{0}\right)=\eta \otimes \nu$ where $\eta \in \mathbb{R}^{n}, \nu \in \mathbb{R}^{N},|\eta|=|\nu|=1$. Without loss of generality, suppose $\nu=e_{1}$. Let $Q=Q(0,1)=\left(-\frac{1}{2}, \frac{1}{2}\right)^{n}$ be the unit cube in $\mathbb{R}^{n}$, so $Q$ has faces either orthogonal or parallel to $e_{1}$. Also, subsequently, we shall let $Q\left(x_{0}, \varrho\right)$ specifically denote the cube $x_{0}+\varrho Q$. Let $\left(r_{k}\right)$ be a sequence decreasing to $0, Q\left(x_{0}, r_{1}\right) \subset \Omega$. Now define the functions $\left(v_{j, k}\right) \subset W^{1, r}\left(Q ; \mathbb{R}^{N}\right)$ by

$$
v_{j, k}(y):=\frac{r_{k}^{n}}{|D u|\left(Q\left(x_{0}, r_{k}\right)\right)}\left(\frac{u_{j}\left(x_{0}+r_{k} y\right)}{r_{k}}-m_{k}\right),
$$

and $\left(v_{k}\right) \subset \mathrm{BV}\left(Q ; \mathbb{R}^{N}\right)$ by

$$
v_{k}(y):=\frac{r_{k}^{n}}{|D u|\left(Q\left(x_{0}, r_{k}\right)\right)}\left(\frac{u\left(x_{0}+r_{k} y\right)}{r_{k}}-m_{k}\right),
$$

where

$$
m_{k}:=\int_{Q} \frac{u\left(x_{0}+r_{k} y\right)}{r_{k}} \mathrm{~d} \mathscr{L}^{n}
$$

Then, by (4.24), $v_{j, k} \stackrel{*}{\rightarrow} v_{k}$ in $\operatorname{BV}(Q ; \Omega)$ as $j \rightarrow \infty$ for each $k$. By Theorem 2.4, we can chose our sequence $\left(r_{k}\right)$ so that $v_{k}$ converges weakly* in $\operatorname{BV}\left(Q ; \mathbb{R}^{N}\right)$ to a function $v \in \mathrm{BV}\left(Q ; \mathbb{R}^{N}\right)$ which can be represented as

$$
v(y)=\psi\left(y_{1}\right) \eta
$$

for a suitable non-decreasing function $\psi:(a, b) \rightarrow \mathbb{R}$. By the same considerations as in Proposition 4.5, the set

$$
\left\{r>0: \mu\left(\partial Q\left(x_{0}, r\right)\right)>0, Q\left(x_{0}, r\right) \subset \Omega\right\}
$$

is at most countable. Hence, by the remark to Theorem 2.4, we can also additionally assume that $\mu\left(\partial Q\left(x_{0}, r_{k}\right)\right)=$ 0 for all $k$. Moreover, for a given $\sigma \in(0,1)$, we have

$$
1 \geq|D v|(Q) \geq|D v|(\sigma \bar{Q}) \geq \sigma^{n}, \quad \lim _{k \rightarrow \infty}\left|D v_{k}\right|(Q) \geq \sigma^{n} .
$$

By Fubini, there exists $s \in(\sigma, 1)$ such that

$$
\lim _{k \rightarrow \infty} \int_{\partial(s Q)}\left|v-v_{k}\right| \mathrm{d} \mathscr{H}^{n-1}=0 .
$$

Now define $\left(w_{k}\right) \subset \operatorname{BV}\left(Q ; \mathbb{R}^{N}\right)$ by

$$
w_{k}:= \begin{cases}v_{k} & \text { on } s Q, \\ v & \text { on } Q \backslash s Q .\end{cases}
$$

Now define the sequence $\left(t_{k}\right)$ converging to $+\infty$ by

$$
t_{k}:=\frac{|D u| Q\left(x_{0}, r_{k}\right)}{r_{k}^{n}} .
$$

By Lemma 4.9 we have

$$
\mathscr{F}_{\mathrm{loc}}\left(w_{k}, Q\right) \geq f(D v(Q)),
$$


so clearly, for fixed $k$,

$$
t_{k}^{-1} \mathscr{F}_{\text {loc }}\left(t_{k} w_{k}, Q\right) \geq t_{k}^{-1} f\left(t_{k} D v(Q)\right) .
$$

Moreover, by the measure representation of $\mathscr{F}_{\text {loc }}$ in Theorem 4.2, we have

$$
t_{k}^{-1} \mathscr{F}_{\mathrm{loc}}\left(t_{k} w_{k}, Q\right) \leq t_{k}^{-1} \mathscr{F}_{\mathrm{loc}}\left(t_{k} v_{k}, s Q\right)+t_{k}^{-1} \mathscr{F}_{\mathrm{loc}}\left(t_{k} w_{k}, Q \backslash \sigma \bar{Q}\right) .
$$

We now obtain various estimates for the terms in (4.30). First note that we have

$$
t_{k}^{-1} \mathscr{F}_{\mathrm{loc}}\left(t_{k} v_{k}, s Q\right) \leq t_{k}^{-1} \mathscr{F}_{\mathrm{loc}}\left(t_{k} v_{k}, Q\right) \leq \liminf _{j \rightarrow \infty} t_{k}^{-1} \int_{Q} f\left(t_{k} \nabla v_{j, k}\right) \mathrm{d} x .
$$

However

$$
\begin{aligned}
& t_{k}^{-1} \int_{Q} f\left(t_{k} \nabla v_{j, k}\right) \mathrm{d} x=\frac{r_{k}^{n}}{|D u| Q\left(x_{0}, r_{k}\right)} \int_{Q} f\left(\nabla u_{j}\left(x_{0}+r_{k} y\right) \mathrm{d} y\right. \\
&=\frac{1}{|D u| Q\left(x_{0}, r_{k}\right)} \int_{Q\left(x_{0}, r_{k}\right)} f\left(\nabla u_{j}(y)\right) \mathrm{d} y \\
& \stackrel{j \rightarrow \infty}{\longrightarrow} \frac{\mu\left(Q\left(x_{0}, r_{k}\right)\right)}{|D u|\left(Q\left(x_{0}, r_{k}\right)\right)},
\end{aligned}
$$

and so

$$
\begin{aligned}
t_{k}^{-1} \mathscr{F}_{\text {loc }}\left(t_{k} v_{k}, s Q\right) & \leq \frac{\mu\left(Q\left(x_{0}, r_{k}\right)\right)}{|D u| Q\left(x_{0}, r_{k}\right)} \\
& \rightarrow \frac{\mathrm{d} \mu}{\mathrm{d}|D u|}\left(x_{0}\right) \quad \text { as } k \rightarrow \infty .
\end{aligned}
$$

Observe that $w_{k}(y) \in \operatorname{span}\{u(z): z \in Q\}$ for all $y \in Q$. Hence we may use the upper bound (3.12) in Theorem 3.5 to obtain

$$
\begin{aligned}
t_{k}^{-1} \mathscr{F}_{\text {loc }}\left(t_{k} w_{k}, Q \backslash \sigma \bar{Q}\right) & \leq t_{k}^{-1} C\left(\mathscr{L}^{n}(Q \backslash \sigma \bar{Q})\right)+\left|t_{k} D w_{k}\right|(Q \backslash \sigma \bar{Q}) \\
& =C\left(t_{k}^{-1}\left(1-\sigma^{n}\right)+\left|D w_{k}\right|(Q \backslash \sigma \bar{Q})\right) .
\end{aligned}
$$

Note that

$$
\left|D w_{k}\right|(Q \backslash \sigma \bar{Q}) \leq|D v|(Q \backslash \sigma \bar{Q})+\left|D v_{k}\right|(Q \backslash \sigma \bar{Q})+\int_{\partial(s Q)}\left|v-v_{k}\right| \mathrm{d} \mathscr{H}^{n-1},
$$

and hence, using (4.27) and (4.28), we have

$$
\limsup _{k \rightarrow \infty}\left|D w_{k}\right|(Q \backslash \sigma \bar{Q}) \leq 2\left(1-\sigma^{n}\right) .
$$

This means

$$
\limsup _{k \rightarrow \infty} t_{k}^{-1} \mathscr{F}_{\text {loc }}\left(t_{k} w_{k}, Q \backslash \sigma \bar{Q}\right) \leq C\left(1-\sigma^{n}\right) .
$$

Lastly, recall the definition of the recession function in (4.1): since $f$ is quasiconvex and hence rank-one convex, we have

$$
\limsup _{t \rightarrow \infty} \frac{f(t \xi)}{t}=\lim _{t \rightarrow \infty} \frac{f(t \xi)}{t}
$$

whenever $\operatorname{rank}(\xi) \leq 1$. Therefore, noting that for $x_{0} \in \operatorname{supp}\left(\left|D^{s} u\right|\right)$ we have $D v(Q)=\eta \otimes e_{1}=\xi\left(x_{0}\right)$, we obtain

$$
\lim _{k \rightarrow \infty} t_{k}^{-1} f\left(t_{k} D v(Q)\right)=f^{\infty}(D v(Q)) .
$$


Also, for such $x_{0}$,

$$
\frac{\mathrm{d} \mu}{\mathrm{d}|D u|}\left(x_{0}\right)=\frac{\mathrm{d} \mu}{\mathrm{d}\left|D^{s} u\right|}\left(x_{0}\right)
$$

Now let $k$ tend to infinity in (4.30), and use (4.31)-(4.33) to get

$$
f^{\infty}\left(\xi\left(x_{0}\right)\right) \leq \frac{\mathrm{d} \mu}{\mathrm{d}\left|D^{s} u\right|}\left(x_{0}\right)+C\left(1-\sigma^{n}\right) .
$$

We conclude the proof by letting $\sigma \nearrow 1$.

We now remove the coercivity condition on $f$ to prove Theorem 4.1.

Proof of Theorem 4.1. By Propositions 4.5 and 4.10, we have established the inequalities (4.7) and (4.8) for when $f$ is coercive, allowing us establish the Theorem in this case. Otherwise, define $f^{\epsilon}: \mathbb{R}^{N \times n} \rightarrow \mathbb{R}$ as

$$
f^{\epsilon}(\xi):=f(\xi)+\epsilon|\xi|,
$$

for all $\xi \in \mathbb{R}^{N \times n}$, for some $\epsilon>0$. Let $\left(u_{j}\right)$ be a sequence in $W^{1, r}\left(\Omega ; \mathbb{R}^{N}\right)$ such that

$$
u_{j} \stackrel{*}{\rightarrow} u \text { in } \operatorname{BV}\left(\Omega ; \mathbb{R}^{N}\right) \text {. }
$$

Then we have

$$
\liminf _{j \rightarrow \infty} \int_{\Omega} f^{\epsilon}\left(\nabla u_{j}(x)\right) \mathrm{d} x \geq \int_{\Omega} f^{\epsilon}(\nabla u(x)) \mathrm{d} x+\int_{\Omega} f_{\infty}^{\epsilon}\left(\frac{D^{s} u}{\left|D^{s} u\right|}\right)\left|D^{s} u\right| .
$$

Now note that

$$
\int_{\Omega} f^{\epsilon}(\nabla u(x)) \mathrm{d} x=\int_{\Omega} f(\nabla u(x)) \mathrm{d} x+\epsilon \int_{\Omega}|\nabla u(x)| \mathrm{d} x
$$

It is also clear that the recession function $f_{\infty}^{\epsilon}$ satisfies

$$
f_{\infty}^{\epsilon}(\xi)=f^{\infty}(\xi)+\epsilon|\xi|,
$$

so

$$
\int_{\Omega} f_{\infty}^{\epsilon}\left(\frac{D^{s} u}{\left|D^{s} u\right|}\right)\left|D^{s} u\right|=\int_{\Omega} f^{\infty}\left(\frac{D^{s} u}{\left|D^{s} u\right|}\right)\left|D^{s} u\right|+\epsilon\left|D^{s} u\right|(\Omega) .
$$

Moreover, since $\left(u_{j}\right)$ is a weakly* convergent sequence in BV, we have

$$
\sup _{j} \int_{\Omega}\left|\nabla u_{j}(x)\right| \mathrm{d} x<\infty .
$$

Therefore we have

$$
\begin{aligned}
\liminf _{j \rightarrow \infty} \int_{\Omega} f\left(\nabla u_{j}(x)\right) \geq & \int_{\Omega} f(\nabla u(x)) \mathrm{d} x+\int_{\Omega} f^{\infty}\left(\frac{D^{s} u}{\left|D^{s} u\right|}\right)\left|D^{s} u\right| \\
& +\epsilon\left(|D u|(\Omega)-\sup _{j} \int_{\Omega}\left|\nabla u_{j}(x)\right| \mathrm{d} x\right),
\end{aligned}
$$

and conclude by letting $\epsilon$ tend to 0 . 


\subsection{Integral representation for certain functions in SBV}

Let us turn our attention again to Lemma 3.3: it is interesting to note that if $u \in \operatorname{SBV}\left(\Omega ; \mathbb{R}^{N}\right)$ satisfies the conditions of this lemma, then the result tells us that $\mathscr{F}_{\text {loc }}(u, \Omega)<\infty$. Hence if we return to the assumption that $r \in\left[1, \frac{n}{n-1}\right)$ then, by Theorem $4.2, \mathscr{F}_{\text {loc }}(u, \cdot)$ is representable by some non-negative, finite Radon measure $\lambda$ on $\Omega$. Moreover, we have

$$
\lambda \ll \mathscr{L}^{n}+\mathscr{H}^{n-1}\left\lfloor J_{u} .\right.
$$

This allows us to refine the upper bound (3.2), and hence get integral represention (instead of a lower bound) in Theorem 4.1 for a certain limited class of funtions in SBV. It makes use of the following corollary of Besicovitch's Covering Theorem, which we state first. For a proof refer to, for example, [22].

Theorem 4.11. Let $\mu$ be a Borel measure on $\mathbb{R}^{n}$ and $\mathcal{B}$ be any collection of nondegenerate closed balls (i.e. balls with radius strictly larger than 0$)$. Let $A$ denote the centres of the balls in $\mathcal{B}$. Assume $\mu(A)<\infty$ and $\inf \{\varrho: B(a, \varrho) \in \mathcal{B}\}=0$ for each $a \in A$. Let $U \subset \mathbb{R}^{n}$ be an open set. Then there exists a countable collection $\mathcal{G}$ of disjoint balls in $\mathcal{B}$ such that

$$
\bigcup_{B \in \mathcal{G}} B \subset U
$$

and

$$
\mu\left((A \cap U) \backslash \bigcup_{B \in \mathcal{G}} B\right)=0 .
$$

Corollary 4.12. Let $\Omega$ be a bounded, open extension domain in $\mathbb{R}^{n}$. Suppose $u \in S B V\left(\Omega ; \mathbb{R}^{N}\right)$ is such that

$$
|\nabla u(x)|=0
$$

for $\mathscr{L}^{n}$-almost all $x \in \Omega$, and that the set $J_{u}$ of approximate jump points of $u$ is the union of finitely many polyhedra. Let $f: \mathbb{R}^{N \times n} \rightarrow \mathbb{R}$ be a continuous function satisfying the growth condition (1.2) for some exponent $1 \leq r<\frac{n}{n-1}$. Let the recession function $f^{\infty}$ be as defined in (4.1), and suppose it is finite on rank-one matrices of the form $u(y) \otimes \nu, y \in \Omega, \nu \in \mathbb{R}^{n}$. Let $\mathscr{F}_{\text {loc }}$ be as defined in (1.4). Then

$$
\mathscr{F}_{\mathrm{loc}}(u, \Omega) \leq \mathscr{L}^{n}(\Omega) f(0)+\int_{\Omega} f^{\infty}\left(\frac{D^{s} u}{\left|D^{s} u\right|}\right)\left|D^{s} u\right| .
$$

Proof of Corollary 4.12. Using Lemma 3.3 and Theorem 4.2, let $\lambda$ be a non-negative finite Radon measure on $\Omega$ representing $\mathscr{F}_{\text {loc }}$, i.e.

$$
\lambda(U)=\mathscr{F} \mathrm{loc}(u, U)
$$

for all open sets $U \subset \Omega$. Now let $\varrho>0$ and let $\mathcal{B}^{(\varrho)}$ be a collection of closed balls of radius at most $\varrho$ that is a fine cover of $\Omega$. Consider any individual ball $B \in \mathcal{B}^{(\varrho)}$. Now take any open ball $B^{\prime} \supset B$ of radius less than $2 \varrho$. If $B^{\prime} \cap J_{u}=\emptyset$, then $D u=\nabla u=0$ on $B^{\prime}$, and $u=a$ for some constant $a \in \mathbb{R}^{N}$. Hence by the definition of $\mathscr{F}_{\text {loc }}$, noting that if $u_{j}=a$ for all $j$, then $u_{j} \stackrel{*}{\rightarrow} u$ in $\operatorname{BV}\left(B^{\prime} ; \mathbb{R}^{N}\right)$,

$$
\lambda\left(B^{\prime}\right)=\mathscr{F}_{\text {loc }}\left(u, B^{\prime}\right) \leq \int_{B^{\prime}} f(0) \mathrm{d} x=\mathscr{L}^{n}\left(B^{\prime}\right) f(0) .
$$

Now suppose $B^{\prime} \cap J_{u} \neq \emptyset$ and $J_{u}$ has only a single polyhedron intersecting with $B^{\prime}$. Then the jump set cuts the ball into two parts $B_{a}^{\prime}$ and $B_{b}^{\prime}$, with (since $\nabla u=0$ )

$$
\begin{cases}u(y)=a & \text { on } B_{a}^{\prime} \\ u(y)=b & \text { on } B_{b}^{\prime}\end{cases}
$$


for some $a, b \in \mathbb{R}^{N}$. Moreover, since any point on a polyhedron is characterised by the intersection of finitely many $n$-1-dimensional hyperplanes, there exists a vector $\nu$, say, such that

$$
\begin{cases}B_{a}^{\prime}=B^{\prime} \cap\left(J_{u}+t \nu\right) & \text { for } t<0 \\ B_{b}^{\prime}=B^{\prime} \cap\left(J_{u}+t \nu\right) & \text { for } t>0\end{cases}
$$

Now let $0<\delta<\varrho$ and define $\chi:(-\varrho, \varrho) \rightarrow \mathbb{R}^{N}$ by

$$
\chi(t):=\left\{\begin{array}{cl}
\left(\frac{b-a}{2 \delta}\right)(x-\delta)+b & \text { if } t \leq-\delta, \\
b & \text { if } t \in(-\delta, \delta),
\end{array}\right.
$$

Now define a function $u_{\delta} \in C\left(B^{\prime} ; \mathbb{R}^{N}\right)$ as follows: note that for each $y \in B^{\prime}$, there exists a unique $t \in(-\varrho, \varrho)$ with $y \in J_{u}+t \nu$, and let

$$
u_{\delta}(y):=\chi(t) .
$$

We therefore have

$$
\nabla u_{\delta}(y)=\left\{\begin{array}{cl}
0 & \text { if } y \in J_{u}+t \nu \text { for } t \notin(-\delta, \delta), \\
\frac{(b-a) \otimes \nu}{2 \delta} & \text { if } y \in J_{u}+t \nu \text { for } t \in(-\delta, \delta) .
\end{array}\right.
$$

Now use the co-area formula to get

$$
\begin{aligned}
\int_{B^{\prime}} f\left(\nabla u_{\delta}\right) \mathrm{d} x & \leq \mathscr{L}^{n}\left(B^{\prime}\right) f(0)+C \int_{-\delta}^{\delta} \int_{\left(J_{u} \cap B^{\prime}\right)+t \nu} f\left(\frac{(b-a) \otimes \nu}{2 \delta}\right) \mathrm{d} \mathscr{H}^{n-1} \mathrm{~d} t \\
& =\mathscr{L}^{n}\left(B^{\prime}\right) f(0)+C \cdot 2 \delta f\left(\frac{(b-a) \otimes \nu}{2 \delta}\right) \times \mathscr{H}^{n-1}\left(J_{u} \cap B^{\prime}\right) \\
& \rightarrow \mathscr{L}^{n}\left(B^{\prime}\right) f(0)+C f^{\infty}((b-a) \otimes \nu) \mathscr{H}^{n-1}\left(J_{u} \cap B^{\prime}\right),
\end{aligned}
$$

as $\delta \rightarrow 0$. Note that the final term here is finite, since $b-a \in \operatorname{span}\{u(y): y \in \Omega\}$. Now take any decreasing sequence $\delta_{j}$ converging to zero, and define $u_{j}$ as $u_{\delta_{j}}$. It is easily verified that $u_{j}$ converges almost everywhere to $u$ in $B^{\prime}$, and that the gradients $\nabla u_{j}$ are bounded in $L^{1}\left(B^{\prime} ; \mathbb{R}^{N}\right)$. Hence, taking a subsequence if necessary, we have $u_{j} \stackrel{*}{\rightarrow} u$ in $\mathrm{BV}\left(B^{\prime} ; \mathbb{R}^{N}\right)$. Thus

$$
\lambda\left(B^{\prime}\right)=\mathscr{F}_{\mathrm{loc}}\left(u, B^{\prime}\right) \leq \mathscr{L}^{n}\left(B^{\prime}\right) f(0)+C f^{\infty}((b-a) \otimes \nu) \mathscr{H}^{n-1}\left(J_{u} \cap B^{\prime}\right) .
$$

Now note that by taking $B^{\prime} \searrow B$ we obtain the inequalities (4.35) and (4.36) for the closed ball $B$.

We also have that $\mathscr{H}^{n-1}$-almost all points $x \in J_{u}$ are on a face of the polyhedron. Hence for balls $B \in \mathcal{B}^{(\varrho)}$ that only intersect with a face, we have that $J_{u}$ is characterised by an $n$-1-dimensional hyperplane passing through $B$. Therefore in this case we may take $\nu$ to be the normal vector of this plane, and $a, b$ are just the one-sided traces $u^{-}(y), u^{+}(y)$ on either side of the jump, for any $y \in B \cap J_{u}$. Moreover, we can take the constant $C$ in (4.36), obtained from the co-area formula used above, to be one. Therefore for such a ball $B$, using the one-homogeneity of $f^{\infty}$, we have

$$
\begin{aligned}
& \lambda(B) \leq \mathscr{L}^{n}(B) f(0)+\int_{J_{u} \cap B} f^{\infty}\left(\left(u^{+}(y)-u^{-}(y)\right) \otimes \nu_{u}(y)\right) \mathrm{d} \mathscr{H}^{n-1}(y) \\
& =\mathscr{L}^{n}(B) f(0)+\int_{J_{u} \cap B} f^{\infty}\left(\frac{u^{+}(y)-u^{-}(y)}{\left|u^{+}(y)-u^{-}(y)\right|} \otimes \nu_{u}(y)\right)\left|u^{+}(y)-u^{-}(y)\right| \mathrm{d} \mathscr{H}^{n-1}(y) \\
& =\mathscr{L}^{n}(B) f(0)+\int_{J_{u} \cap B} f^{\infty}\left(\frac{D^{s} u}{\left|D^{s} u\right|}(y)\right)\left|D^{s} u\right|(y) .
\end{aligned}
$$


Now we apply Theorem 4.11 with $\mu=\mathscr{L}^{n}+\mathscr{H}^{n-1}\left\lfloor J_{u}+\lambda\right.$ and $U=\Omega$. Moreover, we can assume centres of the balls in $\mathcal{B}$, which is a fine partition, is all of $\Omega$. There exists a countable collection of balls $\mathcal{G} \subset \mathcal{B}^{(\varrho)}$ such that

$$
\bigcup_{B \in \mathcal{G}} B \subset U
$$

and

$$
\left(\mathscr{L}^{n}+\mathscr{H}^{n-1}\left\lfloor J_{u}+\lambda\right)\left(\Omega \backslash \bigcup_{B \in \mathcal{G}} B\right)=0\right.
$$

Let $\mathcal{G}_{1}$ denote the set of balls where (4.35) holds and $\mathcal{G}_{2}$ denote the set of balls where (4.37) holds. Then $\mathcal{G} \backslash\left(\mathcal{G}_{1} \cup \mathcal{G}_{2}\right)$ is the set of balls $B$ where $B \cap J_{u}$ is nonempty and not just an $n$-1-dimensional hyperplane. We have already remarked that that $\mathscr{H}^{n-1}$-almost all points $x \in J_{u}$ are locally characterised by a hyperplane, so given $\epsilon>0$, in light of (4.5) we may chose $\varrho$ small enough so that

$$
\sum_{B \in \mathcal{G} \backslash\left(\mathcal{G}_{1} \cup \mathcal{G}_{2}\right)} \lambda(B) \leq \epsilon .
$$

Thus

$$
\begin{aligned}
\lambda(\Omega) & =\sum_{B \in \mathcal{G}} \lambda(B) \\
& =\sum_{B \in \mathcal{G}_{1}} \lambda(B)+\sum_{B \in \mathcal{G}_{2}} \lambda(B)+\epsilon \\
& \leq \sum_{B \in \mathcal{G}_{1}} \mathscr{L}^{n}(B) f(0)+\sum_{B \in \mathcal{G}_{2}}\left(\mathscr{L}^{n}(B) f(0)+\int_{J_{u} \cap B} f^{\infty}\left(\frac{D^{s} u}{\left|D^{s} u\right|}\right)\left|D^{s} u\right|\right)+\epsilon \\
& =\mathscr{L}^{n}(\Omega) f(0)+\int_{J_{u}} f^{\infty}\left(\frac{D^{s} u}{\left|D^{s} u\right|}\right)\left|D^{s} u\right|+\epsilon,
\end{aligned}
$$

from where the required result follows.

The following result follows immediately, combining the upper bound of Corollary 4.12 with the lower bound in Theorem 4.1. Note that for the class of functions $u$ we are considering,

$$
\int_{\Omega} f(\nabla u) \mathrm{d} x=\mathscr{L}^{n}(\Omega) f(0) .
$$

Corollary 4.13. Let $\Omega, u$ be as in Corollary 4.12. Let $f: \mathbb{R}^{N \times n} \rightarrow \mathbb{R}$ be a quasiconvex function satisfying the growth condition (1.2) for $r \in\left[1, \frac{n}{n-1}\right)$. Let the recession function $f^{\infty}$ be as defined in (4.1), and suppose it is finite on rank-one matrices of the form $u(y) \otimes \nu, y \in \Omega, \nu \in \mathbb{R}^{n}$. Then

$$
\mathscr{F}_{\mathrm{loc}}(u, \Omega)=\mathscr{L}^{n}(\Omega) f(0)+\int_{\Omega} f^{\infty}\left(\frac{D^{s} u}{\left|D^{s} u\right|}\right)\left|D^{s} u\right|
$$

\section{Appendix A. Measure representation}

In this appendix, we shall provide a proof of Theorem 4.2. Let $\Omega$ be an open, bounded extension domain in $\mathbb{R}^{n}, n \geq 2$, and let $f$ be a continuous integrand satisfying the growth condition (1.2) where $r \in\left[1, \frac{n}{n-1}\right)$. Consider the Lebesgue-Serrin extensions $\mathscr{F}$ and $\mathscr{F}_{\text {loc }}$ as defined in (1.3) and (1.4) respectively. 
We shall first prove integral estimates for a trace-preserving operator, which will then be used to show that these functionals are representable by finite Radon measures on $\Omega$. This essentially comes directly from the work of Fonseca and Malý in [24], where measure representation is obtained for Lebesgue-Serrin extensions in the context of Sobolev Spaces of exponent larger than one (in fact they consider more general integrands of the form $f=f(x, u, \nabla u))$. The reader may just as easily obtain the results given here by referring to their paper, letting " $p$ " (in their paper) be equal to 1 here, letting their " $q$ " be $r$, and carefully substituting instances of "weak convergence in $W^{1, p}$ " with weak* convergence in BV. However, since this borderline case is not explicitly contained in their paper (and for the convenience of the reader), we give a complete proof here.

These results are important in the context of proving Theorem 4.1, where we obtain a lower semicontinuity result in the case where $f$ is assumed additionally to be quasiconvex and have at most linear growth in certain directions.

\section{A.1. A trace-preserving linear operator}

Adapting a result in [24], we construct a linear operator $T u$ from $W^{1,1}$ into itself that improves integrability over a "layer", allowing us to estimate the $W^{1, r}$ norm of $T u$, for $r \in\left[1, \frac{n}{n-1}\right)$, in terms of a special maximal function. This will be used subsequently to "connect" two functions across a thin transition layer and estimate the increase of energy. In their paper, they are interested specifically in a linear operator from $W^{1, p}$ into $W^{1, p}$ for $p>1$. However, we have observed that the proof also works for $p=1$, which is what we require.

Let $\Omega$ be a bounded, open subset of $\mathbb{R}^{n}$. Let $\eta \in C_{c}^{\infty}(\Omega)$ be a non-negative function and $\left[t_{1}, t_{2}\right] \subset\left(0,\|\eta\|_{\infty}\right)$. Suppose also that $0<|\nabla \eta| \leq A$ on $\left\{t_{1} \leq \eta \leq t_{2}\right\}$. Given a subinterval $(a, b) \subset\left(t_{1}, t_{2}\right)$, let $Z_{a}^{b}$ denote the set $\{a<\eta<b\}$, and for $t_{0} \in\left(t_{1}, t_{2}\right)$, let $\Gamma_{t_{0}}$ denote the level set $\left\{\eta=t_{0}\right\}$.

Fix $t_{0} \in\left(t_{1}, t_{2}\right)$ and note that there exists a diffeomorphism $G_{t_{0}}$ of $\Gamma_{t_{0}} \times\left[t_{1}, t_{2}\right]$ onto $\bar{Z}_{t_{1}}^{t_{2}}$ such that

$$
\left\{\begin{aligned}
G_{t_{0}}\left(z, t_{0}\right) & =z \\
\eta\left(G_{t_{0}}(z, t)\right) & =t
\end{aligned}\right.
$$

for all $z \in G_{t_{0}}, t \in\left[t_{1}, t_{2}\right]$. To see this, consider the flow $h_{z}$ verifying

$$
\left\{\begin{aligned}
\frac{\mathrm{d} h_{z}}{\mathrm{~d} t} & =\frac{\nabla \eta(h(t))}{|\nabla \eta(h(t))|^{2}} \\
h_{z}\left(t_{0}\right) & =z
\end{aligned}\right.
$$

and set $G_{t_{0}}:=h_{z}(t)$. Note that the map $G_{t_{0}}$ is bi-Lipschitz, and also that the Jacobians of $G_{t_{0}}$ and $G_{t_{0}}^{-1}$ are bounded. This allows us to establish the following lemma.

Lemma A.1. [24] Let $s \in\left(t_{1}, t_{2}\right)$ and $\varrho>0$ be such that $[s-\varrho, s+\varrho] \subset\left(t_{1}, t_{2}\right)$. Let $h$ be a non-negative measurable function on $\Omega$. Then

$$
\int_{\{\eta=s\}}\left(\int_{B\left(z, \frac{\rho}{A}\right)} h(y) \mathrm{d} y\right) \mathrm{d} \mathscr{H}^{n-1}(z) \leq C \varrho^{n-1} \int_{Z_{s-\varrho}^{s+\varrho}} h(y) \mathrm{d} y,
$$

where $C$ is a constant dependent on $n, \eta, t_{1}$ and $t_{2}$.

Proof of Lemma A.1. First note that if $z \in \Gamma_{s}$, then $B\left(z, \frac{\varrho}{A}\right) \subset Z_{s-\varrho}^{s+\varrho}$. Hence, using the change of variables $y=G_{s}(z, t)$ and (A.1), we obtain 


$$
\begin{aligned}
& \int_{\{\eta=s\}}\left(\int_{B\left(z, \frac{e}{A}\right)} h(y) \mathrm{d} y\right) \mathrm{d} \mathscr{H}^{n-1}(z)
\end{aligned}
$$

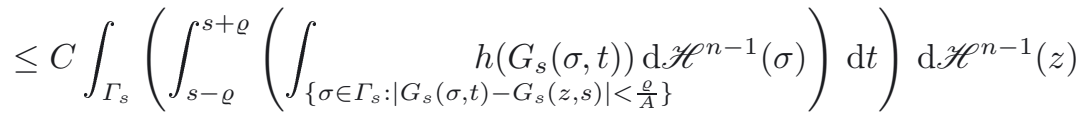

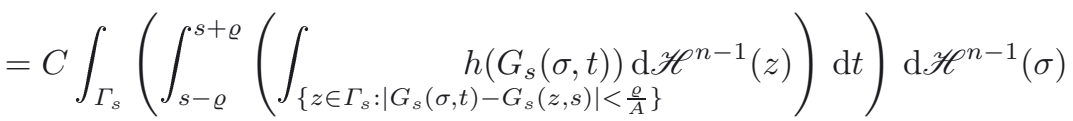

$$
\begin{aligned}
& \leq C \int_{\Gamma_{s} \times(s-\varrho, s+\varrho)} \mathscr{H}^{n-1}\left(\left\{z \in \Gamma_{s}:\left|G_{s}(\sigma, t)-G_{s}(z, s)\right|<\frac{\varrho}{A}\right\}\right) \\
& \times h\left(G_{s}(\sigma, t)\right) \mathrm{d} \mathscr{L}^{n}(\sigma, t) \\
& \leq C \varrho^{n-1} \int_{Z_{s-\varrho}^{s+\varrho}} h(y) \mathrm{d} y,
\end{aligned}
$$

since, due to the Lipschitz continuity of $G_{s}^{-1}$,

$$
\mathscr{H}^{n-1}\left(\left\{z \in \Gamma_{s}:\left|G_{s}(\sigma, t)-G_{s}(z, s)\right|<\frac{\varrho}{A}\right\}\right) \leq C \varrho^{n-1} .
$$

We may now prove the following result.

Lemma A.2. Let $r \in\left[1, \frac{n}{n-1}\right)$. Let $t_{1}<a<b<t_{2}$. There exists a linear operator $T: W^{1,1}\left(\Omega ; \mathbb{R}^{N}\right) \rightarrow$ $W^{1,1}\left(\Omega ; \mathbb{R}^{N}\right)$ such that $T u=u$ on $\Omega \backslash Z_{a}^{b}$ and

$$
\|T u\|_{W^{1, r}\left(Z_{a}^{b}\right)} \leq C(b-a)^{\frac{n}{r}-n+1}\left(\sup _{t \in(a, b)}(t-a)^{-1}\|u\|_{W^{1,1}\left(Z_{a}^{t}\right)}+\sup _{t \in(a, b)}(b-t)^{-1}\|u\|_{W^{1,1}\left(Z_{t}^{b}\right)}\right),
$$

where $C$ depends on $n, r, \eta, t_{1}$ and $t_{2}$.

Proof of Lemma A.2. This proof is directly from [24], but we specifically consider a borderline case that is left out in that proof. Set

$$
T u(x):=f_{B(0,1)} u(x+\theta(x) y) \mathrm{d} y
$$

where

$$
\begin{aligned}
\theta(x):= & \frac{1}{2 A} \max \{0, \min \{\eta(x)-a, b-\eta(x)\}\} \\
= & \left\{\begin{array}{cl}
0 \quad \text { if } \eta(x) \geq b \\
\frac{b-\eta(x)}{2 A} & \text { if } \frac{a+b}{2}<\eta(x)<b \\
\frac{\eta(x)-a}{2 A} & \text { if } a<\eta(x) \leq \frac{a+b}{2} \\
0 & \text { if } \eta(x) \leq a .
\end{array}\right.
\end{aligned}
$$

It is clear to see that $T u(x)=x$ if $x \notin Z_{a}^{b}$, and

$$
T u(x)=f_{B(x, \theta(x))} u(z) \mathrm{d} z
$$


for $x \in Z_{a}^{b}$. Let $c:=\frac{a+b}{2}$ and define

$$
\begin{aligned}
& M_{0}:=\sup _{t \in(a, b)}(t-a)^{-1} \int_{Z_{a}^{t}}|u| \mathrm{d} y, \\
& M_{1}:=\sup _{t \in(a, b)}(t-a)^{-1} \int_{Z_{a}^{t}}|u|+|\nabla u| \mathrm{d} y .
\end{aligned}
$$

First assume $u$ is smooth and fix $\alpha \geq 1$. If $\varrho \in\left(0, \frac{1}{4}(b-a)\right)$ and if $z \in\{\eta=a+2 \varrho\}$, then $\theta(z)=\frac{\varrho}{A}$ and $B(z, \theta(z)) \subset Z_{a+\varrho}^{a+3 \varrho}$. Hence

$$
\begin{aligned}
|T u(z)|^{\alpha} & \leq C \varrho^{-n \alpha}\left(\int_{B\left(z, \frac{\varrho}{A}\right)}|u(y)| \mathrm{d} y\right)^{\alpha} \\
& \leq C \varrho^{-n \alpha}\left(\int_{Z_{a+\varrho}^{a+3 \varrho}}|u(y)| \mathrm{d} y\right)^{\alpha-1}\left(\int_{B\left(z, \frac{\varrho}{A}\right)}|u(y)| \mathrm{d} y\right) .
\end{aligned}
$$

Now use Lemma A.1 to get

$$
\begin{aligned}
& \int_{\{\eta=a+2 \varrho\}}|T u(z)|^{\alpha} \mathrm{d} \mathscr{H}^{n-1}(z) \\
& \leq C \varrho^{-n \alpha}\left(\int_{Z_{a+\varrho}^{a+3 \varrho}}|u(y)| \mathrm{d} y\right)^{\alpha-1} \times \int_{\{\eta=a+2 \varrho\}}\left(\int_{B\left(z, \frac{\varrho}{A}\right)}|u(y)| \mathrm{d} y\right) \mathrm{d} \mathscr{H}^{n-1}(z) \\
& \leq C \varrho^{-n \alpha}\left(\int_{Z_{a+e}^{a+3 \varrho}}|u(y)| \mathrm{d} y\right)^{\alpha-1} \varrho^{n-1}\left(\int_{Z_{a+\varrho}^{a+3 \varrho}}|u(y)| \mathrm{d} y\right) \\
& =C \varrho^{-n \alpha+n-1}\left(\int_{Z_{a+\varrho}^{a+3}}|u(y)| \mathrm{d} y\right)^{\alpha} .
\end{aligned}
$$

By the co-area formula and (A.3) for $\alpha=r$, since $|\nabla \eta|$ is bounded away from zero, we get

$$
\begin{aligned}
\int_{Z_{a}^{c}}|T u(x)|^{r} \mathrm{~d} x & \leq C \int_{0}^{\frac{1}{4}(b-a)}\left(\int_{\{\eta=a+2 \varrho\}}|T u(z)|^{r} \mathrm{~d} \mathscr{H}^{n-1}(z)\right) \mathrm{d} \varrho \\
& \leq C \int_{0}^{\frac{1}{4}(b-a)} \varrho^{-n r+n-1}\left(\int_{Z_{a+\varrho}^{a+3 \varrho}}|u(y)| \mathrm{d} y\right)^{r} \mathrm{~d} \varrho .
\end{aligned}
$$

We have shown that this inequality holds for $u$ when $u$ is smooth. Now we show that (A.4) holds for a general function $u \in L^{1}\left(\Omega ; \mathbb{R}^{N}\right)$. By a standard approximation argument (for example, using mollification) there exists a sequence $\left(u_{j}\right)$ of smooth functions such that $u_{j} \rightarrow u$ strongly in $L^{1}\left(\Omega ; \mathbb{R}^{N}\right)$, and pointwise almost everywhere. 
Now we use this property and Fatou's Lemma to get:

$$
\begin{aligned}
\int_{Z_{a}^{c}}|T u(x)|^{r} \mathrm{~d} x & =\int_{Z_{a}^{c}} \lim _{j \rightarrow \infty}\left|T u_{j}(x)\right|^{r} \mathrm{~d} x \\
& \leq \liminf _{j \rightarrow \infty} \int_{Z_{a}^{c}}\left|T u_{j}(x)\right|^{r} \mathrm{~d} x \\
& \leq \liminf _{j \rightarrow \infty} C \int_{0}^{\frac{1}{4}(b-a)} \varrho^{-n r+n-1}\left(\int_{Z_{a+\varrho}^{a+3 \varrho}}\left|u_{j}(y)\right| \mathrm{d} y\right)^{r} \mathrm{~d} \varrho \\
& =C \int_{0}^{\frac{1}{4}(b-a)} \varrho^{-n r+n-1}\left(\int_{Z_{a+\varrho}^{a+3 \varrho}}|u(y)| \mathrm{d} y\right)^{r} \mathrm{~d} \varrho,
\end{aligned}
$$

as required. Moreover, since

$$
\int_{Z_{a+\varrho}^{a+3 \varrho}}|u(y)| \mathrm{d} y \leq C M_{0} \varrho
$$

we have

$$
\begin{aligned}
\int_{Z_{a}^{c}}|T u(x)|^{r} \mathrm{~d} x & \leq C M_{0}^{r} \int_{0}^{\frac{1}{4}(b-a)} \varrho^{-n r+n-1+r} \mathrm{~d} \varrho \\
& \leq C M_{0}^{r}(b-a)^{n-(n-1) r} .
\end{aligned}
$$

We use an entirely similar argument to conclude that we also have

$$
\int_{Z_{c}^{b}}|T u(x)|^{r} \mathrm{~d} x \leq C M_{0}^{r}(b-a)^{n-(n-1) r} .
$$

Now note that we can also obtain the same estimates with the gradients $\nabla T u$ and $\nabla u$. This is because

$$
\frac{\partial T u}{\partial x_{i}}(x)=f_{B(0,1)}\left(\frac{\partial u}{\partial x_{i}}(x+\theta(x) y)+\sum_{j=1}^{n} \frac{\partial u}{\partial x_{j}}(x+\theta(x) y) y_{j} \frac{\partial \theta}{\partial x_{i}}(x)\right) \mathrm{d} y
$$

and so

$$
|\nabla T u| \leq C T(|\nabla u|)
$$

Therefore the $L^{r}$ estimate (A.5) also holds for derivatives, giving

$$
\|T u\|_{W^{1, r}\left(Z_{a}^{b}\right)} \leq C(b-a)^{\frac{n}{r}-n+1}\left(\sup _{t \in(a, b)}(t-a)^{-1}\|u\|_{W^{1,1}\left(Z_{a}^{t}\right)}+\sup _{t \in(a, b)}(b-t)^{-1}\|u\|_{W^{1,1}\left(Z_{t}^{b}\right)}\right),
$$

as required. 
It remains to show that $T$ is a continuous linear operator. For $u$ smooth, use the co-area formula, (A.3) with $\alpha=1$, and (A.6) to get

$$
\begin{aligned}
\int_{Z_{a}^{c}}(|T u|+|\nabla T u| \mathrm{d} y & \leq C \int_{0}^{\frac{1}{4}(b-a)}\left(\int_{\{\eta=a+2 \varrho\}}|T u(z)|+|\nabla T u(z)| \mathrm{d} \mathscr{H}^{n-1}(z)\right) \mathrm{d} \varrho \\
& \leq C \int_{0}^{\frac{1}{4}(b-a)}\left(\int_{Z_{a+\varrho}^{a+3 \varrho}} \varrho^{-1}(|u(y)|+|\nabla u(y)|) \mathrm{d} y\right) \mathrm{d} \varrho \\
& \leq C \int_{0}^{\frac{1}{4}(b-a)}\left(\int_{a+\varrho}^{a+3 \varrho}\left(\int_{\{\eta=t\}} \varrho^{-1}(|u(z)|+|\nabla u(z)|) \mathrm{d} \mathscr{H}^{n-1}(z)\right) \mathrm{d} t\right) \mathrm{d} \varrho \\
& =C \int_{a}^{b}\left(\int_{\{\eta=t\}}\left(\int_{\frac{t-a}{3}}^{\min \left\{t-a, \frac{b-a}{4}\right\}} \varrho^{-1}(|u(z)|+|\nabla u(z)|) \mathrm{d} \varrho\right) \mathrm{d} \mathscr{H}^{n-1}(z)\right) \mathrm{d} t \\
& \leq C \int_{Z_{a}^{b}}(|u(y)|+|\nabla u(y)|) \mathrm{d} y .
\end{aligned}
$$

A similar bound holds for

$$
\int_{Z_{c}^{b}}(|T u|+|\nabla T u|) \mathrm{d} y
$$

For $u$ smooth, it is easy to see that $T u$ is weakly differentiable and, by the above estimates, that $T u \in$ $W^{1,1}\left(\Omega ; \mathbb{R}^{N}\right)$. For $u \in W^{1,1}\left(\Omega ; \mathbb{R}^{N}\right)$, again let $\left(u_{j}\right)$ be a sequence of smooth functions such that $u_{j} \rightarrow u$ strongly in $W^{1,1}\left(\Omega ; \mathbb{R}^{N}\right)$, and pointwise almost everywhere. By (A.7) and Uniform Boundedness, $\left(T u_{j}\right)$ is bounded in $W^{1,1}\left(\Omega ; \mathbb{R}^{N}\right)$, and hence there exists a subsequence that converges weakly* in $\mathrm{BV}\left(\Omega ; \mathbb{R}^{N}\right)$ to $T u$, so by (A.7) we have

$$
\int_{\Omega}(|T u|+|\nabla T u|) \mathrm{d} y \leq C \int_{\Omega}(|u|+|\nabla u|) \mathrm{d} y,
$$

which establishes that indeed $T$ is a linear continuous map from $W^{1,1}\left(\Omega ; \mathbb{R}^{N}\right)$ into $W^{1,1}\left(\Omega ; \mathbb{R}^{N}\right)$. This completes the proof.

\section{A.2. Technical preliminaries for measure representation}

In this section we establish some results that are key to proving the main result of this section. First, we have the following elementary lemma.

Lemma A.3. Let $\psi$ be a continuous non-decreasing function on an interval $[a, b], a<b$. Then there exist $a^{\prime} \in\left[a, a+\frac{1}{3}(b-a)\right]$ and $b^{\prime} \in\left[b-\frac{1}{3}(b-a), b\right]$ such that $a \leq a^{\prime}<b^{\prime} \leq b$, and

$$
\left\{\begin{array}{l}
\frac{\psi(t)-\psi\left(a^{\prime}\right)}{t-a^{\prime}} \leq 3 \frac{\psi(b)-\psi(a)}{b-a} \\
\frac{\psi\left(b^{\prime}\right)-\psi(t)}{b^{\prime}-t} \leq 3 \frac{\psi(b)-\psi(a)}{b-a}
\end{array}\right.
$$

for all $t \in\left(a^{\prime}, b^{\prime}\right)$.

Proof of Lemma A.3. Without loss of generality we may assume $a=0$ and $\psi(a)=0$. Define

$$
\phi(t):=\psi(t)-3 t \frac{\psi(b)}{b} .
$$

Let $a^{\prime}$ be the point in $[0, b]$ where $\phi$ attains its maximum and let $b^{\prime}$ be the point where $\phi$ attains its minimum. It follows clearly that (A.8) holds from this choice of $a^{\prime}$ and $b^{\prime}$ : note that when $t>\frac{b}{3}$, since $\psi$ is non-decreasing, 
$3 t \frac{\psi(b)}{b}>\psi(b) \geq \psi(b)$. Hence we have $\phi(0)=0$ and $\phi(t)<0$, so it follows that $a^{\prime} \leq \frac{b}{3}$. We argue in a similar way to show that $b^{\prime} \geq b-\frac{1}{3} b$.

We now apply this result to establish the following.

Lemma A.4. Let $V \subset \subset \Omega$ and $W \subset \Omega$ be open sets satisfying $\Omega=V \cup W$. Let $v \in W^{1, r}(V)$ and $w \in W^{1, r}(W)$ for $r \in\left[1, \frac{n}{n-1}\right)$. Let $k \in \mathbb{N}$. Then there exists a function $z \in W_{\text {loc }}^{1, r}(\Omega)$ and open sets $V^{\prime} \subset V$ and $W^{\prime} \subset W$, such that $V^{\prime} \cup W^{\prime}=\Omega, z=v$ on $\Omega \backslash W^{\prime}, z=w$ on $\Omega \backslash V^{\prime}$,

$$
\mathscr{L}^{n}\left(V^{\prime} \cap W^{\prime}\right) \leq C k^{-1}
$$

and

$$
\|z\|_{W^{1, r}\left(V^{\prime} \cap W^{\prime}\right)} \leq C k^{n-1-\frac{n}{r}}\left(\|v\|_{W^{1,1}(V \cap W)}+\|w\|_{W^{1,1}(V \cap W)}+k\|w-v\|_{L^{1}(V \cap W)}\right),
$$

where $C$ is a constant dependent on $r, V$ and $W$.

Proof of Lemma A.4. Let $\eta \in C_{c}^{\infty}(\Omega)$ be such that

$$
\eta=0 \quad \text { on } \Omega \backslash V \text { and } \quad \eta=1 \quad \text { on } \Omega \backslash W .
$$

By Sard's Lemma, the image of the set of all critical points of $\eta$ is a closed set of measure zero. Hence, there exists a nondegenerate interval $[a . b] \subset(0,1) \backslash \eta(\{\nabla \eta=0\})$. Take $k \in \mathbb{N}$ and define

$$
f:=1+|v|+|w|+|\nabla v|+|\nabla w|+k|w-v| .
$$

Since $\{a<\eta<b\} \subset V \cap W$, we may find $j \in\{1, \ldots, k\}$ such that

$$
\int_{\left\{a_{j}<\eta<b_{j}\right\}} f \mathrm{~d} x \leq \frac{1}{k} \int_{V \cap W} f \mathrm{~d} x
$$

where $a_{j}:=a+\frac{(j-1)(b-a)}{k}$ and $b_{j}:=a+\frac{j(b-a)}{k}$. Now apply Lemma A.3 with

$$
\psi(t):=\int_{\{\eta<t\}} f \mathrm{~d} x,
$$

to find $\left[a^{\prime}, b^{\prime}\right] \subset\left[a_{j}, b_{j}\right]$ such that $b^{\prime}-a^{\prime} \geq \frac{1}{3}\left(b_{j}-a_{j}\right)$, and

$$
\begin{aligned}
& \int_{\left\{a^{\prime}<\eta<t\right\}} f \mathrm{~d} x \leq 3 \frac{t-a^{\prime}}{b^{\prime}-a^{\prime}} \int_{\left\{a^{\prime}<\eta<b^{\prime}\right\}} f \mathrm{~d} x, \\
& \int_{\left\{t<\eta<b^{\prime}\right\}} f \mathrm{~d} x \leq 3 \frac{b^{\prime}-t}{b^{\prime}-a^{\prime}} \int_{\left\{a^{\prime}<\eta<b^{\prime}\right\}} f \mathrm{~d} x
\end{aligned}
$$

for all $t \in\left(a^{\prime}, b^{\prime}\right)$. Now set

$$
V^{\prime}:=\Omega \cap\left\{\eta>a^{\prime}\right\}, \quad W^{\prime}:=\Omega \cap\left\{\eta<b^{\prime}\right\},
$$

and

$$
u:=\left\{\begin{array}{cl}
v & \text { on }\left\{\eta \geq b^{\prime}\right\}, \\
\frac{\left(\eta-a^{\prime}\right) v+\left(b^{\prime}-\eta\right) w}{b^{\prime}-a^{\prime}} & \text { on }\left\{a^{\prime} \leq \eta \leq b^{\prime}\right\}, \\
w & \text { on }\left\{\eta \leq a^{\prime}\right\} .
\end{array}\right.
$$

By (A.11), it is clear that $V^{\prime} \subset V, W^{\prime} \subset W$, and $V^{\prime} \cup W^{\prime}=\Omega$. Moreover, (A.9) holds as $|\nabla \eta|$ is bounded away from zero on $\{a<\eta<b\}$ and $b^{\prime}-a^{\prime} \leq \frac{b-a}{k}$. It is easy to verify that on $\left\{a^{\prime}<\eta<b^{\prime}\right\}$ we have

$$
|u|+|\nabla u| \leq C f .
$$

Now use (A.12), (A.13) and Lemma A.2 to find a function $z \in W^{1,1}(\Omega)$ such that $z=u=v$ on $\left\{\eta \geq b^{\prime}\right\}=\Omega \backslash W^{\prime}$, $z=u=w$ on $\left\{\eta \leq a^{\prime}\right\}=\Omega \backslash V^{\prime}$, and (A.10) is satisfied. 


\section{A.3. Proof of measure representation}

We first recall some key definitions. Let $\mu$ be a Radon measure on $\bar{\Omega}$, where $\Omega$ is a bounded, open subset of $\mathbb{R}^{n}$. Then we say that $\mu$ (strongly) represents $\mathscr{F}(u, \cdot)$ if

$$
\mu(U)=\mathscr{F}(u, U)
$$

for all open sets $U \subset \Omega$. We say that $\mu$ weakly represents $\mathscr{F}(u, \cdot)$ if

$$
\mu(U) \leq \mathscr{F}(u, U) \leq \mu(\bar{U})
$$

for all open sets $U \subset \Omega$. The following two theorems are the main results of this section. Note that the first one is Theorem 4.2 .

Theorem A.5. Let $f: \mathbb{R}^{N \times n} \rightarrow \mathbb{R}$ be a continuous function satisfying the growth condition (1.2) for some exponent $1 \leq r<\frac{n}{n-1}$. Let $u \in B V\left(\Omega ; \mathbb{R}^{N}\right)$ and $\mathscr{F}_{\text {loc }}$ be as defined in (1.4). Then if $\mathscr{F}_{\text {loc }}(u, \Omega)<\infty$, then there exists a non-negative, finite Radon measure $\lambda$ on $\Omega$ which represents $\mathscr{F}_{\text {loc }}$.

Theorem A.6. Let $f: \mathbb{R}^{N \times n} \rightarrow \mathbb{R}$ be a continuous function satisfying the growth condition (1.2) for some exponent $1 \leq r<\frac{n}{n-1}$. Let $u \in B V\left(\Omega ; \mathbb{R}^{N}\right)$ and $\mathscr{F}$ be as defined in (1.3). Then if $\mathscr{F}(u, \Omega)<\infty$, then there exists a non-negative, finite Radon measure $\mu$ on $\bar{\Omega}$ which weakly represents $\mathscr{F}$.

The following lemma is instrumental in our proofs of these two theorems.

Lemma A.7. Let $f: \mathbb{R}^{N \times n} \rightarrow \mathbb{R}$ be a continuous function satisfying the growth condition (1.2) for some exponent $1 \leq r<\frac{n}{n-1}$. Let $V, W \subset \Omega$ be open sets, $V \subset \subset \Omega$ and $\Omega=V \cup W$, and let $u \in W^{1,1}\left(\Omega ; \mathbb{R}^{N}\right)$. Let $\mathscr{F}$ be as defined in (1.3). Then

$$
\mathscr{F}(u, \Omega) \leq \mathscr{F}(u, V)+\mathscr{F}(u, W)
$$

An identical assertion holds for $\mathscr{F}_{\text {loc }}$ as defined in (1.4).

Proof of Lemma A.7. Let $\epsilon>0$. By the definition of $\mathscr{F}$, there exist sequences $\left(v_{k}\right) \subset W^{1, r}\left(V ; \mathbb{R}^{N}\right)$ and $\left(w_{k}\right) \subset$ $W^{1, r}\left(W ; \mathbb{R}^{N}\right)$ such that

$$
v_{k} \stackrel{*}{\rightarrow} u \text { weakly* in } \operatorname{BV}\left(V ; \mathbb{R}^{N}\right) \text { and } w_{k} \stackrel{*}{\rightarrow} u \text { weakly* in } \operatorname{BV}\left(W ; \mathbb{R}^{N}\right),
$$

and (by eliminating the first terms of the sequences if necessary),

$$
\begin{gathered}
\int_{V} f\left(\nabla v_{k}\right) \mathrm{d} x \leq \mathscr{F}(u, V)+\epsilon, \\
\int_{W} f\left(\nabla w_{k}\right) \mathrm{d} x \leq \mathscr{F}(u, W)+\epsilon .
\end{gathered}
$$

Moreover, by taking subsequences if necessary, we can ensure

$$
\left\|v_{k}-u\right\|_{L^{1}(V \cap W)} \leq \frac{1}{k} \quad \text { and } \quad\left\|w_{k}-u\right\|_{L^{1}(V \cap W)} \leq \frac{1}{k}
$$

for all $k$. Using Lemma A.4, for each $k$ we can find open sets $V_{k} \subset V, W_{k} \subset W$, and functions $\left(z_{k}\right) \subset$ $W^{1, r}\left(\Omega ; \mathbb{R}^{N}\right)$, such that $V_{k} \cup W_{k}=\Omega, z_{k}=v_{k}$ on $\Omega \backslash W_{k}$, and $z_{k}=w_{k}$ on $\Omega \backslash V_{k}$. Moreover, by growth condition (1.2), (A.14), and since by the Uniform Boundedness Principle the sequences $\left(v_{k}\right),\left(w_{k}\right)$ are bounded in $W^{1,1}\left(V ; \mathbb{R}^{N}\right), W^{1,1}\left(W ; \mathbb{R}^{N}\right)$ respectively,

$$
\begin{aligned}
& \int_{V_{k} \cap W_{k}} f\left(\nabla z_{k}\right) \mathrm{d} x \leq L \int_{V_{k} \cap W_{k}}\left(1+\left|\nabla z_{k}\right|^{r}\right) \mathrm{d} x \\
& \quad \leq C k^{-1}+C k^{r(n-1)-n}\left(\|v\|_{W^{1,1}(V \cap W)}+\|w\|_{W^{1,1}(V \cap W)}+k\|w-v\|_{L^{1}(V \cap W)}\right)^{r} \\
& \quad \leq C k^{r(n-1)-n} .
\end{aligned}
$$


Therefore

$$
\int_{\Omega} f\left(\nabla z_{k}\right) \mathrm{d} x \leq \int_{V} f\left(\nabla v_{k}\right) \mathrm{d} x+\int_{W} f\left(\nabla w_{k}\right) \mathrm{d} x+C k^{r(n-1)-n} .
$$

Now we show that $z_{k} \stackrel{*}{\rightarrow} u$ in $\operatorname{BV}\left(\Omega ; \mathbb{R}^{N}\right)$. Certainly, since $z_{k}=v_{k}$ on $\Omega \backslash W_{k}, z_{k}=w_{k}$ on $\Omega \backslash V_{k}$, and by (A.15) the $W^{1, r}$-norm of each $z_{k}$ is bounded on $V_{k} \cap W_{k}$, the sequence is bounded in $W^{1,1}\left(\Omega ; \mathbb{R}^{N}\right)$. Moreover, using the fact that $\mathscr{L}^{n}\left(V_{k} \cap W_{k}\right) \rightarrow 0$ and Rellich-Kondrachov, we have that each subsequence of $\left(z_{k}\right)$ has a subsubsequence converging in $L^{1}\left(\Omega ; \mathbb{R}^{N}\right)$ to $u$. Therefore it follows that $z_{k} \stackrel{*}{\rightarrow} u$ in $\operatorname{BV}\left(\Omega ; \mathbb{R}^{N}\right)$ as required. Hence by the definition of $\mathscr{F}$ and (A.16)

$$
\mathscr{F}(u, \Omega) \leq \liminf _{k \rightarrow \infty} \int_{\Omega} f\left(\nabla z_{k}\right) \mathrm{d} x \leq \mathscr{F}(u, V)+\mathscr{F}(u, W)+2 \epsilon,
$$

which concludes the proof. The proof for $\mathscr{F}_{\text {loc }}$ is essentially the same.

Proof of Theorem A.6. First we assume in addition that $f$ satisfies the coercivity condition

$$
f(\xi) \geq c_{0}|\xi|
$$

for some constant $c_{0}>0$, for all $\xi \in \mathbb{R}^{N \times n}$. Using Proposition 3.2 , let $\left(u_{k}\right) \subset W^{1, r}\left(\Omega ; \mathbb{R}^{N}\right)$ be a minimising sequence for $\mathscr{F}(u, \Omega)$, i.e. $u_{k} \stackrel{*}{\rightarrow} u$ in $\operatorname{BV}\left(\Omega ; \mathbb{R}^{N}\right)$ and

$$
\lim _{k \rightarrow \infty} \int_{\Omega} f\left(\nabla u_{k}\right) \mathrm{d} x=\mathscr{F}(u, \Omega) .
$$

Note that since the sequence $f\left(\nabla u_{j}\right) \mathscr{L}^{n}$ is bounded in $\mathscr{M}(\bar{\Omega})$, we that have for some subsequence (for convenience not relabelled) there exists a measure $\mu$ in $\bar{\Omega}$ such that

$$
f\left(\nabla u_{j}\right) \stackrel{*}{\rightarrow} \mu \text { in } \mathscr{M}(\bar{\Omega}) .
$$

Clearly, since $f$ is non-negative, $\mu$ must also be a non-negative measure on $\bar{\Omega}$. In particular, we have

$$
\mu(\bar{\Omega})=\mathscr{F}(u, \Omega)
$$

and for every open set $V \subset \Omega$

$$
\mathscr{F}(u, V) \leq \liminf _{k \rightarrow \infty} \int_{V} f\left(\nabla u_{k}\right) \mathrm{d} x \leq \mu(\bar{V}) .
$$

Now let $V \subset \Omega$ be an open set and fix $\epsilon>0$. Take an open set $Z \subset \subset V$ such that

$$
\mu(V)-\mu(Z)<\epsilon .
$$

Now use Lemma A.7, (A.18) and (A.19) to get

$$
\begin{aligned}
\mu(V) \leq \mu(Z)+\epsilon & =\mu(\bar{\Omega})-\mu(\bar{\Omega} \backslash Z)+\epsilon \\
& \leq \mathscr{F}(u, \Omega)-\mathscr{F}(u, \bar{\Omega} \backslash Z)+\epsilon \\
& \leq \mathscr{F}(u, V)+\epsilon .
\end{aligned}
$$

Let $\epsilon \rightarrow 0$ to obtain

$$
\mu(V) \leq \mathscr{F}(u, V) .
$$

Now we show how the coercivity assumption (A.17) may be removed. Define $f^{\epsilon}: \mathbb{R}^{N \times n} \rightarrow \mathbb{R}$ as

$$
f^{\epsilon}(\xi):=f(\xi)+\epsilon|\xi|,
$$


for all $\xi \in \mathbb{R}^{N \times n}$, for some $\epsilon>0$. Define $\mathscr{F}^{\epsilon}$ to be the corresponding Lebesgue-Serrin extension of $f^{\epsilon}$ as in (1.3). By the above part of the proof, we obtain a measure $\mu^{\epsilon}$ weakly representing $\mathscr{F} \epsilon$. Letting $\left(u_{k}\right) \subset W^{1, r}\left(\Omega ; \mathbb{R}^{N}\right)$ be a minimising sequence for $\mathscr{F}^{\epsilon}(u, \Omega)$, we have

$$
\mu^{\epsilon}(\bar{\Omega})=\mathscr{F}^{\epsilon}(u, \Omega) \leq \mathscr{F}(u, \Omega)+\epsilon \sup _{k}\left\|u_{k}\right\|_{W^{1,1}} \leq C .
$$

Moreover, if $U \subset \Omega$ is open, then clearly

$$
\mathscr{F}(u, U) \leq \liminf _{k \rightarrow \infty} \int_{U} f\left(\nabla u_{k}\right) \mathrm{d} x \leq \liminf _{k \rightarrow \infty} \int_{U} f^{\epsilon}\left(\nabla u_{k}\right) \mathrm{d} x \leq \mu^{\epsilon}(\bar{U}) .
$$

Hence, by (A.20), we may select $\epsilon_{j} \rightarrow 0$ such that the sequence $\mu^{\epsilon_{j}}$ converges weakly* in the sense of measures to a finite, non-negative, Radon measure $\mu$. Then, by (A.21),

$$
\mathscr{F}(u, U) \leq \mu^{\epsilon_{j}}(\bar{U}),
$$

and passing to the weak* limit,

$$
\mathscr{F}(u, U) \leq \mu(\bar{U}) .
$$

Conversely, let $\epsilon^{\prime}>0$, and take a sequence $\left(v_{k}\right) \subset W^{1, r}\left(U ; \mathbb{R}^{N}\right)$ satisfying $v_{k} \stackrel{*}{\rightarrow} u$ weakly* in $\mathrm{BV}\left(U ; \mathbb{R}^{N}\right)$ and

$$
\int_{U} f\left(\nabla v_{k}\right) \mathrm{d} x \leq \mathscr{F}(u, U)+\epsilon^{\prime}
$$

for all $k$. Then, for $j$ large enough, we have

$$
\int_{U} f^{\epsilon_{j}}\left(\nabla v_{k}\right) \mathrm{d} x=\int_{U}\left(f\left(\nabla v_{k}\right)+\epsilon_{j}\left|v_{k}\right|+\epsilon_{j}\left|\nabla v_{k}\right|\right) \mathrm{d} x \leq \mathscr{F}(u, U)+2 \epsilon^{\prime},
$$

and so

$$
\mu^{\epsilon_{j}}(U) \leq \mathscr{F}^{\epsilon_{j}}(u, U) \leq \liminf _{k \rightarrow \infty} \int_{U} f^{\epsilon_{j}}\left(\nabla v_{k}\right) \mathrm{d} x \leq \mathscr{F}(u, U)+2 \epsilon^{\prime} .
$$

Now we pass to the weak* limit and let $\epsilon^{\prime} \rightarrow 0$ to conclude the proof.

Now we show that we also have strong measure representation for $\mathscr{F}$ if certain technical conditions are satisfied. First we establish the following lemma, which will also play a part in our proof of Theorem A.5.

Lemma A.8. Let $f: \mathbb{R}^{N \times n} \rightarrow \mathbb{R}$ be a continuous function satisfying the growth condition (1.2) for some exponent $1 \leq r<\frac{n}{n-1}$. Let $u \in B V\left(\Omega ; \mathbb{R}^{N}\right)$ and $\mathscr{F}$ be as defined in (1.3). Let $U$ be an open subset of $\Omega$. If $\mu$ is a Radon measure on $\bar{\Omega}$ weakly representing $\mathscr{F}(u, \cdot)$ and

$$
\inf _{K}\{\mathscr{F}(u, U \backslash K): K \subset U \text { is compact }\}=0,
$$

then

$$
\mu(U)=\mathscr{F}(u, U) .
$$

An identical statement holds for $\mathscr{F}_{\text {loc }}$ as defined in (1.4).

Proof of Lemma A.8. We need to show $\mathscr{F}(u, U) \leq \mu(U)$. Let $\epsilon>0$ and, using (A.22), let $K \subset U$ be a compact set such that

$$
\mathscr{F}(u, U \backslash K)<\epsilon .
$$


Now take an open set $W$ such that $K \subset W \subset \subset U$ and apply Lemma A.7 to get

$$
\begin{aligned}
\mathscr{F}(u, U) & \leq \mathscr{F}(u, W)+\mathscr{F}(u, U \backslash K) \\
& \leq \mathscr{F}(u, W)+\epsilon \\
& \leq \mu(\bar{W})+\epsilon \\
& \leq \mu(U)+\epsilon .
\end{aligned}
$$

Take $\epsilon \rightarrow 0$ to complete the proof. The proof for $\mathscr{F}_{\text {loc }}$ is the same.

This allows us to deduce

Corollary A.9. Let $f: \mathbb{R}^{N \times n} \rightarrow \mathbb{R}$ be a continuous function satisfying the growth condition (1.2) for some exponent $1 \leq r<\frac{n}{n-1}$. Let $u \in B V\left(\Omega ; \mathbb{R}^{N}\right)$ and $\mathscr{F}$ be as defined in (1.3). If $\mu$ is a finite Radon measure on $\bar{\Omega}$ weakly representing $\mathscr{F}(u, \cdot)$, then $\mu$ represents $\mathscr{F}(u, \cdot)$ if and only if there exists a Radon measure $\nu$ such that

$$
\mathscr{F}(u, U) \leq \nu(U)
$$

for all open subsets $U \subset \Omega$.

Proof of Corollary A.9. If (A.23) is satisfied, then clearly (A.22) holds for any open set $U \subset \Omega$ so, by Lemma A.8, $\mu$ represents $\mathscr{F}(u, \cdot)$. The converse implication is trivial, taking $\nu=\mu$.

We are now in a position to prove the remaining main theorem of this section.

Proof of Theorem A.5. Again, assume first that the coercivity condition (A.17) is satisfied. Using the proof of Theorem A.6, there exists a Radon measure $\lambda$ on $\bar{\Omega}$ such that for every open set $U \subset \Omega$,

$$
\lambda(U) \leq \mathscr{F}_{\mathrm{loc}}(u, U) \leq \lambda(\bar{U}) .
$$

For a given open set $U \subset \Omega$, we shall show additionally that

$$
\lambda(U) \geq \mathscr{F}_{\mathrm{loc}}(u, U) .
$$

Take an increasing sequence of open, bounded, smooth sets $U_{j} \subset \subset U, j \in \mathbb{N}$, such that $\bar{U}_{j} \subset U_{j+1}$ for all $j$ and $U=\bigcup_{j=1}^{\infty} U_{j}$. By the definition of $\mathscr{F}_{\text {loc }}$, for each $j \geq 3$ there exists a sequence $\left(u_{j, k}\right) \subset W_{\text {loc }}^{1, r}\left(U_{j} \backslash \bar{U}_{j-2} ; \mathbb{R}^{N}\right)$ such that

$$
u_{j, k} \stackrel{*}{\rightarrow} u \text { weakly* in } \mathrm{BV}\left(U_{j} \backslash \bar{U}_{j-2} ; \mathbb{R}^{N}\right) \text { as } k \rightarrow \infty,
$$

and

$$
\int_{U_{j} \backslash \bar{U}_{j-2}} f\left(\nabla u_{j, k}\right) \mathrm{d} x \leq \mathscr{F}_{\text {loc }}\left(u, U_{j} \backslash \bar{U}_{j-2}\right)+2^{-j}
$$

Fix positive integers $\alpha_{j}$, which will be determined later, and note that by taking a subsequence (for convenience not relabelled) we may assume $u_{j, k} \rightarrow u$ almost everywhere in $U_{j} \backslash \bar{U}_{j-2}$ as $k \rightarrow \infty$, and

$$
\left\|u_{j, k}-u\right\|_{L^{1}\left(U_{j} \backslash \bar{U}_{j-2}\right)} \leq 2^{-j-k} \alpha_{j}^{-1} .
$$

Now use Lemma A.4 to connect $u_{j, k}$ to $u_{j+1, k}$ across $U_{j} \backslash \bar{U}_{j-1}$. There exist open sets $V_{j, k}^{+}, V_{j+1, k}^{-}$such that

$$
\left\{\begin{array}{l}
V_{j, k}^{+} \subset U_{j} \backslash \bar{U}_{j-2} \\
V_{j+1, k}^{-} \subset U_{j+1} \backslash \bar{U}_{j-1} \\
U_{j+1} \backslash \bar{U}_{j-2}=V_{j, k}^{+} \cup V_{j+1, k}^{-} \\
\mathscr{L}^{n}\left(V_{j, k}^{+} \cap V_{j+1, k}^{-}\right) \leq C_{j} 2^{-j-k} \alpha_{j}^{-1},
\end{array}\right.
$$


and there exist functions $\left(z_{j, k}\right) \subset W^{1, r}\left(U_{j+1} \backslash \bar{U}_{j-2} ; \mathbb{R}^{N}\right)$ such that

$$
z_{j, k}=\left\{\begin{array}{cl}
u_{j, k} & \text { on }\left(U_{j} \backslash \bar{U}_{j-2}\right) \backslash V_{j+1, k}^{-}, \\
u_{j+1, k} & \text { on }\left(U_{j+1} \backslash \bar{U}_{j-1}\right) \backslash V_{j, k}^{+}
\end{array}\right.
$$

and

$$
\begin{aligned}
\int_{V_{j, k}^{+} \cap V_{j+1, k}^{-}} & f\left(\nabla z_{j, k}\right) \mathrm{d} x \leq L \int_{V_{j, k}^{+} \cap V_{j+1, k}^{-}}\left(1+\left|\nabla z_{j, k}\right|^{r}\right) \mathrm{d} x \\
\leq & L C_{j} 2^{-j-k} \alpha_{j}^{-1}+C_{j}\left(2^{j+k} \alpha_{j}\right)^{r(n-1)-n}\left(\left\|u_{j, k}\right\|_{W^{1,1}\left(U_{j} \backslash \bar{U}_{j-1}\right)}\right. \\
& \left.+\left\|u_{j+1, k}\right\|_{W^{1,1}\left(U_{j} \backslash \bar{U}_{j-1}\right)}+2^{j+k} \alpha_{j}\left\|u_{j+1, k}-u_{j, k}\right\|_{L^{1}\left(U_{j} \backslash \bar{U}_{j-1}\right)}\right)^{r} \\
\leq & C_{j}\left(2^{j+k} \alpha_{j}\right)^{r(n-1)-n},
\end{aligned}
$$

where $C_{j}$ is a constant depending on $j$. Hence we may specify our choice of $\alpha_{j}$ so that $\alpha_{j}^{r(n-1)-n} C_{j} \leq 1$. Now define $\left(z_{k}\right) \subset W_{\text {loc }}^{1, r}\left(\Omega \backslash U_{1} ; \mathbb{R}^{N}\right)$ by

$$
z_{k}:=\left\{\begin{array}{cl}
z_{j, k} & \text { on } V_{j, k}^{+} \cap V_{j+1, k}^{-}, \\
u_{j+1, k} & \text { on }\left(U_{j+1} \backslash U_{j-1}\right) \backslash\left(V_{j, k}^{+} \cup V_{j+2, k}^{-}\right) .
\end{array}\right.
$$

Now fix $m \in \mathbb{N}, m \geq 2$. We have

$$
\begin{aligned}
\int_{U \backslash \bar{U}_{m}} f\left(\nabla z_{k}\right) \mathrm{d} x \leq & \sum_{j=m+1}^{\infty} \int_{U_{j} \backslash \bar{U}_{j-1}} f\left(\nabla z_{k}\right) \mathrm{d} x \\
\leq & \sum_{j=m+1}^{\infty}\left(\int_{U_{j+1} \backslash \bar{U}_{j-1}} f\left(\nabla u_{j+1, k}\right) \mathrm{d} x\right. \\
& \left.+\int_{U_{j} \backslash \bar{U}_{j-1}} f\left(\nabla u_{j, k}\right) \mathrm{d} x+\int_{V_{j, k}^{+} \cap V_{j+1, k}^{-}} f\left(\nabla z_{j, k}\right) \mathrm{d} x\right) \\
\leq & \sum_{j=m+1}^{\infty}\left(2 \mathscr{F}_{\text {loc }}\left(u, U_{j+1} \backslash \bar{U}_{j-1}\right)+2^{-j+1}+2^{(j+k)(r(n-1)-n)}\right) \\
\leq & \sum_{j=m+1}^{\infty}\left(2 \lambda\left(U_{j+2} \backslash U_{j-1}\right)+2^{-j+1}+2^{(j+k)(r(n-1)-n)}\right) \\
\leq & 6 \lambda\left(U \backslash U_{m-1}\right)+2^{-m+1}+2^{k(r(n-1)-n)} \sum_{j=m+1}^{\infty}\left(2^{n-r(n-1)}\right)^{-j} \\
\leq & 6 \lambda\left(U \backslash U_{m-1}\right)+2^{-m+1}+2^{k(r(n-1)-n)} \cdot o(m) .
\end{aligned}
$$

By the coercivity condition (A.17) and the above, we have

$$
\begin{aligned}
\int_{U \backslash \bar{U}_{m}}\left|\nabla z_{k}\right| & \leq C \int_{\Omega \backslash \bar{U}_{m}} f\left(\nabla z_{k}\right) \mathrm{d} x \\
& \leq C 6 \lambda\left(U \backslash U_{m-1}\right)+C \\
& \leq C 6 \mathscr{F}_{\text {loc }}\left(u, U \backslash U_{m-1}\right)+C
\end{aligned}
$$


for all $k$ so, since $\mathscr{F}_{\text {loc }}(u, \Omega)<\infty$, the sequence $\left(z_{k}\right)$ is bounded in $W^{1,1}\left(U \backslash \bar{U}_{m} ; \mathbb{R}^{N}\right)$. Now note that

$$
\begin{aligned}
\mathscr{L}^{n}\left(\bigcup_{j=1}^{\infty}\left(V_{j, k}^{+} \cap V_{j+1, k}^{-}\right)\right) & \leq \sum_{j=1}^{\infty} \mathscr{L}^{n}\left(V_{j, k}^{+} \cap V_{j+1, k}^{-}\right) \\
& \leq \sum_{j=1}^{\infty} C_{j} 2^{-j-k} \alpha_{j}^{-1} \\
& \leq \sum_{j=1}^{\infty} 2^{-j-k} \\
& \rightarrow 0 \quad \text { as } k \rightarrow \infty .
\end{aligned}
$$

Hence, arguing using Rellich-Kondrachov as in Lemma A.7, we have that $z_{k} \stackrel{*}{\rightarrow} u$ in $\operatorname{BV}\left(U \backslash \bar{U}_{m} ; \mathbb{R}^{N}\right)$. Therefore

$$
\mathscr{F}_{\mathrm{loc}}\left(u, U \backslash \bar{U}_{m}\right) \leq 6 \lambda\left(U \backslash U_{m-1}\right)+2^{-m+1},
$$

and so

$$
\begin{aligned}
\inf _{K}\{\mathscr{F}(u, U \backslash K): K \subset U \text { is compact }\} & \leq \lim _{m \rightarrow \infty} \mathscr{F}_{\operatorname{loc}}\left(u, U \backslash \bar{U}_{m}\right) \\
& \leq \lim _{m \rightarrow \infty}\left(6 \lambda\left(U \backslash U_{m-1}\right)+2^{-m+1}\right) \\
& =0 .
\end{aligned}
$$

Thus condition (A.22) of Lemma A.8 is satisfied, allowing us to conclude that indeed

$$
\lambda(U)=\mathscr{F}_{\mathrm{loc}}(u, U) .
$$

We remove the coercivity assumption (A.17) using the same argument as in the proof of Theorem A.6.

Acknowledgements. The author is thankful for the support of the Oxford Centre for Nonlinear PDE (OxPDE) through the EPSRC Science and Innovation award EP/E035027/1, and of the Ludwig Maximilians University, Munich. He is also grateful to Jan Kristensen for numerous helpful discussions, and an anonymous referee for reading a preliminary version of the manuscript and providing thorough feedback with many useful comments.

\section{REFERENCES}

[1] E. Acerbi and N. Fusco, Semicontinuity problems in the calculus of variations. Arch. Ration. Mech. Anal. 86 (1984) $125-145$.

[2] G. Alberti, Rank one property for derivatives of functions with bounded variation. Proc. Roy. Soc. Edinburgh Sect. A 123 (1993) 239-274.

[3] G. Alberti and L. Ambrosio, A geometrical approach to monotone functions in $\mathbf{R}^{n}$, Math. Z. 230 (1999) 259-316.

[4] M. Amar and V. De Cicco, Quasi-polyhedral approximation of BV-functions. Ric. Mat. 54 (2005) 485-490 (2006).

[5] L. Ambrosio, A compactness theorem for a new class of functions of bounded variation. Boll. Un. Mat. Ital. B 3 (1989) $857-881$.

[6] L. Ambrosio, Existence theory for a new class of variational problems. Arch. Ration. Mech. Anal. 111 (1990) $291-322$.

[7] L. Ambrosio, On the lower semicontinuity of quasiconvex integrals in $\operatorname{SBV}\left(\Omega, \mathbf{R}^{k}\right)$. Nonlinear Anal. 23 (1994) $405-425$.

[8] L. Ambrosio and G. Dal Maso, On the relaxation in $\operatorname{BV}\left(\Omega ; \mathbf{R}^{m}\right)$ of quasi-convex integrals. J. Funct. Anal. 109 (1992) $76-97$.

[9] L. Ambrosio, N. Fusco and J. Hutchinson, Higher integrability of the gradient and dimension of the singular set for minimisers of the Mumford-Shah functional. Calc. Var. Partial Differ. Eq. 16 (2003) 187-215.

[10] L. Ambrosio, N. Fusco, and D. Pallara, Functions of bounded variation and free discontinuity problems. Oxf. Math. Monogr. The Clarendon Press Oxford University Press, New York (2000).

[11] L. Ambrosio, S. Mortola, and V. Tortorelli, Functionals with linear growth defined on vector valued BV functions. J. Math. Pures Appl. 70 (1991) 269-323.

[12] L. Ambrosio and D. Pallara, Integral representations of relaxed functionals on $\mathrm{BV}\left(\mathbf{R}^{n}, \mathbf{R}^{k}\right)$ and polyhedral approximation. Indiana Univ. Math. J. 42 (1993) 295-321. 
[13] P. Aviles and Y. Giga, Variational integrals on mappings of bounded variation and their lower semicontinuity. Arch. Ration. Mech. Anal. 115 (1991) 201-255.

[14] J. Ball and F. Murat, $W^{1, p}$-quasiconvexity and variational problems for multiple integrals. J. Funct. Anal. 58 (1984) $225-253$.

[15] G. Bouchitté, I. Fonseca, and J. Malý, The effective bulk energy of the relaxed energy of multiple integrals below the growth exponent. Proc. Roy. Soc. Edinburgh Sect. A 128 (1998) 463-479.

[16] A. Braides and A. Coscia, The interaction between bulk energy and surface energy in multiple integrals. Proc. Roy. Soc. Edinburgh Sect. A 124 (1994) 737-756.

[17] G. Buttazzo, Semicontinuity, relaxation and integral representation in the calculus of variations. Pitman Res. Notes in Math. Ser., vol. 207. Longman Scientific \& Technical, Harlow (1989).

[18] L. Carbone and R. De Arcangelis, Further results on $\Gamma$-convergence and lower semicontinuity of integral functionals depending on vector-valued functions. Ric. Mat. 39 (1990) 99-129.

[19] G. Dal Maso, Integral representation on $\operatorname{BV}(\Omega)$ of $\Gamma$-limits of variational integrals. Manuscr. Math. 30 (1979/80) 387-416.

[20] E. De Giorgi and L. Ambrosio, New functionals in the calculus of variations. Atti Accad. Naz. Lincei Rend. Cl. Sci. Fis. Mat. Natur. 82 (1988) 199-210 (1989).

[21] E. De Giorgi, F. Colombini, and L. Piccinini, Frontiere orientate di misura minima e questioni collegate. Scuola Normale Superiore, Pisa (1972).

[22] L. Evans and R. Gariepy, Measure theory and fine properties of functions. Stud.Adv. Math. CRC Press, Boca Raton, FL (1992).

[23] I. Fonseca, Lower semicontinuity of surface energies. Proc. Roy. Soc. Edinburgh Sect. A 120 (1992) 99-115.

[24] I. Fonseca and J. Malý, Relaxation of multiple integrals below the growth exponent. Annal. Inst. Henri Poincaré Anal. Non Linéaire 14 (1997) 309-338.

[25] I. Fonseca and P. Marcellini, Relaxation of multiple integrals in subcritical Sobolev spaces. J. Geom. Anal. 7 (1997) 57-81.

[26] I. Fonseca and S. Müller, Quasi-convex integrands and lower semicontinuity in L1. SIAM J. Math. Anal. 23 (1992) $1081-1098$.

[27] I. Fonseca and S. Müller, Relaxation of quasiconvex functionals in $\operatorname{BV}\left(\Omega, \mathbf{R}^{p}\right)$ for integrands $f(x, u, \nabla u)$. Arch. Ration. Mech. Anal. 123 (1993) 1-49.

[28] I. Fonseca and P. Rybka, Relaxation of multiple integrals in the space $\operatorname{BV}\left(\Omega, \mathbf{R}^{p}\right)$. Proc. Roy. Soc. Edinburgh Sect. A 121 (1992) 321-348.

[29] C. Goffman and J. Serrin, Sublinear functions of measures and variational integrals. Duke Math. J. 31 (1964) $159-178$.

[30] J. Kristensen, Lower semicontinuity of quasi-convex integrals in $\operatorname{BV}\left(\Omega ; \mathbf{R}^{m}\right)$. Calc. Var. Partial Differ. Eqs. 7 (1998) $249-261$.

[31] C. Larsen, Quasiconvexification in $W^{1,1}$ and optimal jump microstructure in BV relaxation. SIAM J. Math. Anal. 29 (1998) $823-848$.

[32] H. Lebesgue, Intégrale, longueur, aire. Ann. Mat. Pura Appl. 7 (1902) 231-359.

[33] J. Malý, Weak lower semicontinuity of polyconvex and quasiconvex integrals. Manuscr. Math. 85 (1994) 419-428.

[34] P. Marcellini, Approximation of quasiconvex functions, and lower semicontinuity of multiple integrals. Manuscr. Math. 51 (1985) 1-28.

[35] P. Marcellini, On the definition and the lower semicontinuity of certain quasiconvex integrals. Annal. Inst. Henri Poincaré Anal. Non Linéaire 3 (1986) 391-409.

[36] P. Mattila, Geometry of sets and measures in Euclidean spaces. Cambridge Stud. Adv. Math., vol. 44. Cambridge University Press, Cambridge (1995), Fractals and rectifiability.

[37] N. Meyers, Quasi-convexity and lower semi-continuity of multiple variational integrals of any order. Trans. Amer. Math. Soc. 119 (1965) 125-149.

[38] C. Morrey, Quasi-convexity and the lower semicontinuity of multiple integrals. Pacific J. Math. 2 (1952) $25-53$.

[39] C. Morrey, Multiple integrals in the calculus of variations. Classics Math. (1966).

[40] S. Müller, On quasiconvex functions which are homogeneous of degree 1. Indiana Univ. Math. J. 41 (1992) $295-301$.

[41] J. Reshetnyak, General theorems on semicontinuity and convergence with functionals. Sibirsk. Mat. Ž. 8 (1967) $1051-1069$.

[42] F. Rindler, Lower semicontinuity and Young measures in $\operatorname{BV}\left(\Omega ; \mathbf{R}^{m}\right)$ without Alberti's Rank-One Theorem. Adv. Calc. Var. 5 (2012) 127-159.

[43] W. Rudin, Real and complex analysis, 3rd edition, McGraw-Hill Book Co., New York (1987).

[44] T. Schmidt, Regularity of relaxed minimizers of quasiconvex variational integrals with $(p, q)$-growth. Arch. Ration. Mech. Anal. 193 (2009) 311-337.

[45] T. Schmidt, A simple partial regularity proof for minimizers of variational integrals. NoDEA Nonlinear Differ. Eq. Appl. 16 (2009) 109-129.

[46] J. Serrin, A new definition of the integral for nonparametric problems in the calculus of variations. Acta Math. 102 (1959) 23-32.

[47] J. Serrin, On the definition and properties of certain variational integrals. Trans. Amer. Math. Soc. 101 (1961) $139-167$.

[48] P. Soneji, Lower semicontinuity in BV of quasiconvex integrals with subquadratic growth. ESAIM: COCV 19 (2013) 555-573.

[49] W. Ziemer, Weakly differentiable functions. Sobolev spaces and functions of bounded variation. Graduate Texts Math., vol. 120. Springer-Verlag, New York (1989). 